\title{
Métodos de predição para modelo logístico misto com $k$ efeitos aleatórios
}

\author{
Karin Ayumi Tamura
}

\author{
TESE APRESENTADA \\ $\mathrm{AO}$ \\ Instituto DE MATEMÁticA E Estatística \\ DA \\ Universidade De SÃo Paulo \\ PARA \\ OBTENÇÃO DO TÍTULO \\ $\mathrm{DE}$ \\ DouTOR EM CIÊNCIAS \\ Programa: Estatística \\ Orientadora: Prof ${ }^{\mathrm{a}}$. Dr ${ }^{\mathrm{a}}$. Vivivana Giampaoli
}

São Paulo, 17 de dezembro de 2012 


\section{Métodos de predição para modelo logístico misto $\operatorname{com} k$ efeitos aleatórios}

Esta versão da tese contêm as correções e alterações sugeridas pela Comissão Julgadora durante a defesa da versão original do trabalho, realizada em 17/12/2012. Uma cópia da versão original está disponível no Instituto de Matemática e Estatística da Universidade de São Paulo.

Comissão Julgadora:

- Prof ${ }^{a}$. Dra ${ }^{\mathrm{a}}$. Viviana Giampaoli (orientadora) - IME-USP

- Prof ${ }^{\mathrm{a}}$. Dr ${ }^{\mathrm{a}}$. Denise Aparecida Botter - IME-USP

- Prof. Dr. Francisco José de Azevêdo Cysneiros - UFPE

- Prof ${ }^{\mathrm{a}}$ Dra $^{\mathrm{a}}$. Maria Del Pilar Diaz - UNC-Argentina

- Prof $^{a}$ Dr$^{\mathrm{a}}$. Cibele Maria Russo Noveli - ICMC-USP 


\section{Agradecimentos}

Primeiramente, agradeço aos meus pais, Luiz e Yoshiko, por terem me preparado, me apoiado e me proporcionado a oportunidade de chegar até aqui. Agradeço também à minha família, em especial à minha irmã Larissa que me auxiliou na revisão do texto da tese.

Obrigada aos professores Gilberto A. Paula, Denise A. Botter, Francisco J.A. Cysneiros, Maria Del Pilar Diaz e Cibele M.R. Noveli pelas enriquecedoras sugestões fornecidas para o presente trabalho.

Aos meus companheiros(as) da empresa e da USP, em especial aos meus chefes Rubens Stephan e Marcelo Sousa, que sempre me incentivaram durante todo o curso de pós-graduação. Agradeço aos meus colegas de trabalho Eduardo Taniguchi e Caio Soares por terem me auxiliado na construção do banco de dados de aplicação.

Por fim, agradeço à duas pessoas que tiveram papel fundamental para que o trabalho de doutorado fosse finalizado com sucesso.

A primeira pessoa seria minha orientadora, Viviana Giampaoli, que acreditou no meu potencial e me orientou impecavelmente como professora e amiga durante todo o curso. Obrigada pela paciência, dedicação, e profissionalismo.

A outra pessoa, a qual dedico este trabalho, é meu marido Alexandre Noma. Além ser um amigo e companheiro, compreendeu minha ausência e me apoiou em todas as etapas do doutorado. Obrigada por todo seu amor, e saiba que este sentimento é que me motiva a querer ser uma pessoa cada vez melhor. 


\section{Resumo}

TAMURA, K. A. Métodos de predição para modelo logístico misto com $k$ efeitos aleatórios. 2012. 106 f. Tese (Doutorado) - Instituto de Matemática e Estatística, Universidade de São Paulo, São Paulo, 2012.

A predição de uma observação futura para modelos mistos é um problema que tem sido extensivamente estudado. Este trabalho trata o problema de atribuir valores para os efeitos aleatórios e/ou variável resposta de novos grupos para o modelo logístico misto, cujo objetivo é predizer respostas futuras com base em parâmetros estimados previamente. Na literatura, existem alguns métodos de predição para este modelo que considera apenas o intercepto aleatório. Para a regressão logística mista com $k$ efeitos aleatórios, atualmente não há métodos propostos para a predição dos efeitos aleatórios de novos grupos. Portanto, foram propostas novas abordagens baseadas no método da média zero, no melhor preditor empírico (MPE), na regressão linear e nos modelos de regressão não-paramétricos. Todos os métodos de predição foram avaliados usando os seguintes métodos de estimação: aproximação de Laplace, quadratura adaptativa de Gauss-Hermite e quaseverossimilhança penalizada. Os métodos de estimação e predição foram analisados por meio de estudos de simulação, com base em sete cenários, com comparações de diferentes valores para: o tamanho de grupo, os desvios-padrão dos efeitos aleatórios, a correlação entre os efeitos aleatórios, e o efeito fixo. Os métodos de predição foram aplicados em dois conjuntos de dados reais. Em ambos os problemas os conjuntos de dados apresentaram estrutura hierárquica, cujo objetivo foi predizer a resposta para novos grupos. Os resultados indicaram que o método MPE apresentou o melhor desempenho em termos de predição, entretanto, apresentou alto custo computacional para grandes bancos de dados. As demais metodologias apresentaram níveis de predição semelhantes ao MPE, e reduziram drasticamente o esforço computacional.

Palavras-chave: predição, modelo logístico misto, efeitos aleatórios. 


\section{Abstract}

TAMURA, K. A. Prediction methods for mixed logistic regression with $k$ random effects. 2012. 106 f. Tese (Doutorado) - Instituto de Matemática e Estatística, Universidade de São Paulo, São Paulo, 2012.

The prediction of a future observation in a mixed regression is a problem that has been extensively studied. This work treat the problem of assigning the random effects and/or the outcome of new groups for the mixed logistic regression, in which the aim is to predict future outcomes based on the parameters previously estimated. In the literature, there are some prediction methods for this model that considers only the random intercept. For the mixed logistic regression with $k$ random effects, there is currently no method for predicting the random effects of new groups. Therefore, we proposed new approaches based on average zero method, empirical best predictor (EBP), linear regression and nonparametric regression models. All prediction methods were evaluated by using the estimation methods: Laplace approximation, adaptive Gauss-Hermite quadrature and penalized quasi-likelihood. The estimation and prediction methods were analyzed by simulation studies, based on seven simulation scenarios, which considered comparisons of different values for: the group size, the standard deviations of the random effects, the correlation between the random effects, and the fixed effect. The prediction methods were applied in two real data sets. In both problems the data set presented hierarchical structure, and the objective was to predict the outcome for new groups. The results indicated that EBP presented the best performance in prediction terms, however it has been presented high computational cost for big data sets. The other methodologies presented similar level of prediction in relation to EBP, and drastically reduced the computational effort.

Keywords: prediction, mixed logistic model, random effects. 


\section{Sumário}

Lista de Abreviaturas $\quad$ iii

Lista de Figuras $\quad$ iv

Lista de Tabelas

1 Introdução $\quad 1$

1.1 Conceitos Preliminares . . . . . . . . . . . . . . . . . . . 1

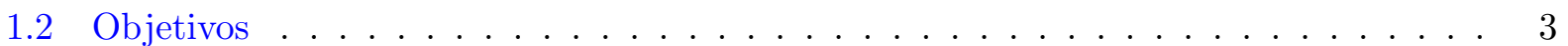

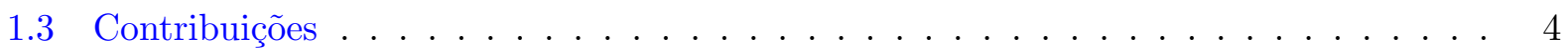

1.4 Organização do Trabalho . . . . . . . . . . . . . . . . . . . 4

2 Trabalhos Relacionados $\quad 6$

2.1 Modelo linear normal . . . . . . . . . . . . . . . . . . . . 6

2.2 Modelos lineares generalizados . . . . . . . . . . . . . . . . 8

2.2 .1 Modelo de regressão logística . . . . . . . . . . . . . . . . . . . . . 9

2.3 Métodos de predição para modelos mistos com intercepto aleatório . . . . . . . . . 10

2.3.1 Método de Predição MPLNVE para o modelo linear misto . . . . . . . . . . . 10

2.3.2 Método de Predição MPE para o MLGM . . . . . . . . . . . . . . . . . . 11

2.3.3 Método de Predição MPE para o modelo logístico misto . . . . . . . . . . . . 14

2.4 Medidas de desempenho para o modelo com resposta binária . . . . . . . . . 16

2.4 .1 AUC e curva ROC . . . . . . . . . . . . . . . . . . . . . . . . . . . . . . . . .

2.4 .2 Estatística $\mathrm{KS} \ldots \ldots \ldots \ldots \ldots \ldots$

3 Modelo logístico misto com $k$ efeitos aleatórios: Métodos de Estimação e Predição 21

3.1 Modelo logístico misto . . . . . . . . . . . . . . . . . . . . . . 21

3.2 Métodos de estimação . . . . . . . . . . . . . . . . . . . 23

3.2 .1 Aproximação de Laplace $(\mathrm{AL}) \quad \ldots \ldots \ldots$. . . . . . . . . . . . 23

3.2.2 Quadratura Adaptativa de Gauss-Hermite (QAGH) . . . . . . . . . . . . . . 25

3.2.3 Quase-Verossimilhança Penalizada (QVP) . . . . . . . . . . . . . . 27

3.3 Métodos de Predição . . . . . . . . . . . . . . . . . . . . . . 31

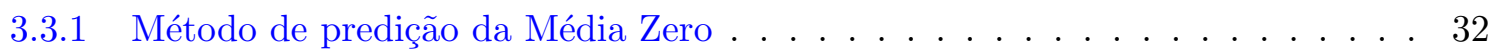

3.3 .2 Método de predição MPE . . . . . . . . . . . . . . . . . . 33

3.3.3 Método de predição via modelos de regressão . . . . . . . . . . . . . . . . 37 
4 Estudos de simulação $\quad 46$

4.1 Esquema de simulação . . . . . . . . . . . . . . . . . . . . . 48

4.2 Análise dos resultados . . . . . . . . . . . . . . . . . . . . . 49

4.2.1 Estimativa dos parâmetros . . . . . . . . . . . . . . . 50

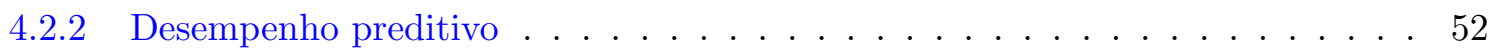

4.2 .3 Resíduos dos valores preditos . . . . . . . . . . . . . 56

4.2.4 Discussão do uso dos métodos de predição . . . . . . . . . . . . . . . . 58

4.3 Aspectos Computacionais . . . . . . . . . . . . . . . . . 59

4.3.1 Custo computacional dos métodos de estimação e predição . . . . . . . . . . 60

5 Aplicações $\quad \mathbf{6 2}$

5.1 Aplicação em Dados Longitudinais: Modelo para avaliar desnutrição grave . . . . . . 64

5.1 Estimativas dos Parâmetros . . . . . . . . . . . . . . 66 66

5.1 .2 Desempenho preditivo . . . . . . . . . . . . . . . 67

5.2 Aplicação em Marketing: Modelo de venda de um produto adicional . . . . . . . . 69

5.2.1 Estimativas dos Parâmetros . . . . . . . . . . . . . . . . . 72

5.2 .2 Desempenho preditivo . . . . . . . . . . . . . . . 74

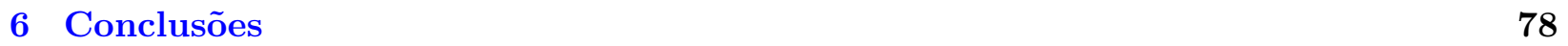

6.1 Considerações Finais . . . . . . . . . . . . . . . . . . . . 78

6.2 Sugestões para Pesquisas Futuras . . . . . . . . . . . . . . . . . . 80

$\begin{array}{ll}\text { A Bloxplos dos estudos de simulação } & 81\end{array}$

Referências Bibliográficas $\quad 90$ 


\section{Lista de Abreviaturas}

$\begin{array}{ll}\text { AUC } & \text { Area Under the Curve (Área abaixo da curva ROC) } \\ \text { AL } & \text { Aproximação de Laplace } \\ \text { BA } & \text { Base de Ajuste } \\ \text { BE } & \text { Bayes Empírico } \\ \text { BP } & \text { Base de Predição } \\ \text { BLUP } & \text { Best Linear Unbiased Predictor } \\ \text { EBLUP } & \text { Empirical Best Linear Unbiased Predictor } \\ \text { EQM } & \text { Erro Quadrátido Médio } \\ \text { Md.res.qua } & \text { Mediana dos Resíduos ao Quadrado } \\ \text { Md.res.abs } & \text { Mediana dos Resíduos Absolutos } \\ \text { MLG } & \text { Modelo Linear Generalizado } \\ \text { MLGM } & \text { Modelo Linear Generalizado Misto } \\ \text { MMLG } & \text { Modelo Multinível Linear Generalizado } \\ \text { MP } & \text { Melhor Preditor } \\ \text { MPE } & \text { Melhor Preditor Empírico } \\ \text { MPLNV } & \text { Melhor Preditor Linear Não Viesado } \\ \text { MPLNVE } & \text { Melhor Preditor Linear Não Viesado Empírico } \\ \text { MZ } & \text { Média Zero } \\ \text { NP } & \text { Não-paramétrico } \\ \text { QVP } & \text { Quase-Verossimilhança Penalizada } \\ \text { QAGH } & \text { Quadratura Adaptativa de Gauss-Hermite } \\ \text { ROC } & \text { Receiver Operating Characteristics (Curva ROC) } \\ \text { RL } & \text { Regressão Linear }\end{array}$




\section{Lista de Figuras}

2.1 Comparação entre os modelos de regressão tradicional e o misto. $\quad$. . . . . . . . . . 7

2.2 Curva ROC - figura ilustrativa. . . . . . . . . . . . . . . . . . . . 18

3.1 Resumo do método de predição via modelos de regressão. . . . . . . . . . . . . . . 38

5.1 Estrutura hierárquica do banco de dados de desnutrição. . . . . . . . . . . . . . . 64

5.2 Exemplo da estruturação do banco de dados de desnutrição. . . . . . . . . . . . . . 65

5.3 Amostra de ajuste e predição dos dados de desnutrição. . . . . . . . . . . . . . . 65

5.4 Método de Estimação Laplace dos dados de desnutrição: (a) valores estimados do intercepto aleatório por criança; (b) valores estimados da inclinação aleatória por criança; (c) gráfico de dispersão dos efeitos aleatórios; (d) qq-plot normal do intercepto aleatório; (e) qq-plot normal da inclinação aleatória, na BA. . . . . . . . . . . 67

5.5 Estrutura hierárquica do banco de dados de telefonia. . . . . . . . . . . . . . . 69

5.6 Base de ajuste (junho/2010) e predição (dezembro/2010) dos dados de telefonia. . . 71

5.7 Medidas de desempenho AUC e KS na base de predição para novos grupos, por método de predição para os dados de telefonia. . . . . . . . . . . . . . . . 75

5.8 Medidas de desempenho AUC e KS na base de predição (todos: novos e antigos), por método de predição para os dados de telefonia. . . . . . . . . . . . . 76

5.9 Método de Estimação Laplace dos dados de telefonia: (a) valores estimados do intercepto aleatório por cliente; (b) valores estimados da inclinação aleatória por cliente; (c) gráfico de dispersão dos efeitos aleatórios; (d) qq-plot normal do intercepto aleatório; (e) qq-plot normal da inclinação aleatória, na BA. . . . . . . . . . . 77

A.1 Bloxplot das réplicas para Cenário 1 na base de predição: Distribuição das medianas dos resíduos ao quadrado, distribuição das medianas dos resíduos absolutos, distribuição do AUC e distribuição do KS. . . . . . . . . . . . . . . . . .

A.2 Bloxplot das réplicas para Cenário 2 na base de predição: Distribuição das medianas dos resíduos ao quadrado, distribuição das medianas dos resíduos absolutos, distribuição do AUC e distribuição do KS. . . . . . . . . . . . . . . . . .

A.3 Bloxplot das réplicas para Cenário 3 na base de predição: Distribuição das medianas dos resíduos ao quadrado, distribuição das medianas dos resíduos absolutos, distribuição do AUC e distribuição do KS. . . . . . . . . . . . . . . . . .

A.4 Bloxplot das réplicas para Cenário 4 na base de predição: Distribuição das medianas dos resíduos ao quadrado, distribuição das medianas dos resíduos absolutos, distribuição do AUC e distribuição do KS. . . . . . . . . . . . . . . . . . 86 
A.5 Bloxplot das réplicas para Cenário 5 na base de predição: Distribuição das medianas dos resíduos ao quadrado, distribuição das medianas dos resíduos absolutos, distribuição do AUC e distribuição do KS. . . . . . . . . . . . . . . . . . . . 87

A.6 Bloxplot das réplicas para Cenário 6 na base de predição: Distribuição das medianas dos resíduos ao quadrado, distribuição das medianas dos resíduos absolutos, distribuição do AUC e distribuição do KS. . . . . . . . . . . . . . . . . 88

A.7 Bloxplot das réplicas para Cenário 7 na base de predição: Distribuição das medianas dos resíduos ao quadrado, distribuição das medianas dos resíduos absolutos, distribuição do AUC e distribuição do KS. . . . . . . . . . . . . . . . . . . . 89 


\section{Lista de Tabelas}

2.1 Tabela de classificação - resposta observada versus resposta predita. . . . . . . . . . 17

4.1 Cenários de simulação. . . . . . . . . . . . . . . . . . . . . . 46

4.2 Média (percentual de variação em relação ao verdadeiro valor) das estimativas dos parâmetros $\beta, \sigma_{1}, \sigma_{2}$ e $\sigma_{12}$, por método de estimação, baseado no resultado de 1.000

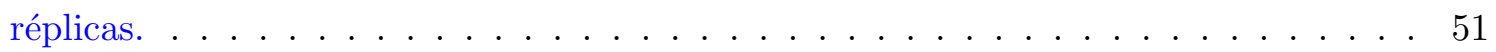

4.3 Média de AUC e KS para modelo tradicional e misto baseado em 1.000 réplicas, por método de estimação e predição, na base de ajuste e predição. . . . . . . . . . . . 53

4.4 Md.res.qua e Md.res.abs na BP, por método de estimação e predição. . . . . . . . . . 57

4.5 Número de problemas ocorridos na estimação dos parâmetros do modelo misto até se completar as 1.000 réplicas desejadas. . . . . . . . . . . . . . . . . . 60

5.1 Estimativas dos parâmetros do modelo tradicional e misto para os dados de desnutrição. 66

5.2 Medidas de desempenho dos métodos de predição (tradicional, MPE, RL, NP e MZ), na BP para os dados de desnutrição. . . . . . . . . . . . . . . . . . . . 68

5.3 Medidas Resumo para os dados de telefonia: Quantidade de terminais por cliente

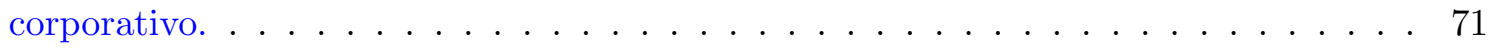

5.4 Anova do modelo tradicional e misto para os dados de telefonia: Análise do p-valor. 72

5.5 Estimativas dos parâmetros, erro padrão e p-valor para modelo tradicional e misto para os dados de telefonia. . . . . . . . . . . . . . . . . 73

5.6 Variáveis presentes nos modelos RL e NP para variável resposta intercepto aleatório, para os dados de telefonia. . . . . . . . . . . . . . . . . . . 74

5.7 Variáveis presentes nos modelos RL e NP para variável resposta inclinação aleatória, para os dados de telefonia. . . . . . . . . . . . . . . . . . . . 75 


\section{Capítulo 1}

\section{Introdução}

Neste capítulo, a primeira seção apresenta os conceitos preliminares que são importantes para contextualizar o tema abordado no presente do trabalho. As seções posteriores apresentam o objetivo, as principais contribuições e a organização da tese.

\subsection{Conceitos Preliminares}

A crescente popularidade dos modelos multiníveis deve-se principalmente à larga abrangência de aplicações em diversos campos científicos para problemas com estrutura hierárquica nos dados.

Quando os dados estão estruturados de forma hierárquica, as unidades de um mesmo nível, agrupadas em uma unidade de nível mais alto, são raramente independentes. Isto acontece porque essas unidades compartilham um mesmo ambiente ou apresentam características semelhantes.

Podem-se citar exemplos como uma pesquisa na área de ciências sociais com o objetivo de investigar como o ambiente de trabalho influencia na produtividade dos funcionários, ou um estudo na área de marketing com o interesse em investigar como as informações de uso do celular dos funcionários de uma empresa estão relacionados ao cancelamento das respectivas linhas telefônicas. Para ambos os exemplos, os dados são provenientes de uma mesma estrutura hierárquica, com funcionários agrupados dentro de empresas. Nestes exemplos, existe o interesse em mensurar as variáveis nos níveis: trabalhador e firma. Para acomodar este tipo de estrutura de dados, os modelos multiníveis têm a flexibilidade de combinar informações em diferentes níveis hierárquicos, por meio de um sistema hierárquico de equações de regressão, possibilitando a inclusão de variáveis em ambos os níveis (individual e grupal), sendo as observações individuais hierarquizadas dentro de um grupo.

Em modelos multiníveis, os grupos são representados por efeitos aleatórios, e sua variabilidade entre-grupos é representada por meio da variabilidade do intercepto aleatório e/ou da variabilidade da inclinação aleatória.

Referências na literatura sobre os modelos multiníveis podem ser vistos nos livros Hox (1995) e Goldstein (2011), por exemplo. Os modelos multiníveis também são conhecidos como modelos hierárquicos, conforme Raudenbush e Bryk (2001). Uma das principais características dessa classe de modelos é que a mesma possibilita considerar vários níveis hierárquicos. Considere um exemplo 
com quatro níveis: alunos que estão dentro de uma sala de aula, que por sua vez, estão dentro de uma escola, e estas escolas estão dentro de uma determinada região; sendo seus respectivos níveis de hierarquia: aluno, sala de aula, escola e região.

Neste trabalho, considerou-se um caso particular do modelo multinível, conhecido na literatura como modelo misto, que é o modelo multinível restrito a dois níveis hierárquicos. A classe de modelos mistos abrange os modelos lineares generalizados mistos (MLGM), que é uma extensão dos modelos lineares generalizados (MLG), proposto por McCullagh e Nelder (1989), conforme Demidenko (2004). Os MLGM foram considerados com a mesma abordagem adotada por Pinheiro e Bates (2000), isto é, o modelo misto sendo um caso particular do modelo multinível, apesar de Renard (2002) considerar os modelos multiníveis como um caso particular dos modelos mistos. Uma característica importante dos MLGM é que esta classe de modelos incorpora efeitos aleatórios que são estimados individualmente para cada grupo.

O objetivo desta tese é apresentar propostas diferentes de predição para o modelo logístico misto, que é um modelo particular da classe dos MLGM. A contribuição das propostas de predição torna-se relevante quando se tem o objetivo de predizer o valor do efeito aleatório ou da variável resposta das observações de um novo grupo, uma vez que não se conhece o valor individual de seus efeitos aleatórios.

Para predição de um modelo com resposta normal, nos quais os modelos lineares mistos são apropriados, Prasad e Rao (1990) propuseram o método do melhor preditor linear não viesado empírico (MPLNVE) para predizer observações futuras, focando na aplicação em pequenas áreas.

Contribuições em predição para o caso do modelo logístico misto foram apresentadas em Jiang e Lahiri (2001), Skrondal e Rabe-Hesketh (2009) e Tamura e Giampaoli (2010). Nesses artigos, os modelos consideraram apenas um efeito aleatório, sendo este o intercepto. Jiang e Lahiri (2001) propuseram uma solução para predizer a reposta e o efeito aleatório de um novo grupo (no nível do grupo), focando na aplicação em pequenas áreas. Skrondal e Rabe-Hesketh (2009) compararam métodos diferentes para predizer o efeito aleatório e discutiram vários tipos de predição: de uma nova observação em um grupo hipotético, de um novo grupo e de um grupo existente. Em outra abordagem, Tamura e Giampaoli (2010) apresentaram um método para predizer a resposta de uma observação dentro de um novo grupo (no nível da observação), comparando o desempenho em termo de predição do modelo logístico tradicional em relação ao modelo logístico misto.

Antes de prosseguir, é importante salientar a diferença entre os termos estimação e predição dos efeitos aleatórios. A distinção destes dois conceitos torna-se mais clara voltando para o exemplo na área de marketing: se existe um grupo de clientes que já cancelaram suas linhas telefônicas, parece razoável descrever a "probabilidade de cancelamento" dos celulares com base nas informações de uso do telefone como um problema de estimação. Por outro lado, se o interesse é atribuir a probabilidade de cancelamento a um cliente recém-chegado à empresa de telefonia com o objetivo de evitar o cancelamento futuro, isto é um problema de predição.

Em modelos de regressão, geralmente, tem-se o interesse em estudar a relação das variáveis preditoras em relação à variável resposta. A variável resposta é o "objetivo a ser modelado"e as 
variáveis preditoras são aquelas que explicam ou se relacionam com a variável resposta. Desta forma, quando for considerado um modelo de regressão, o objetivo é a busca da relação esquematizada por

$$
Y=X+\epsilon
$$

em que $Y$ é a variável resposta, $X$ é um conjunto de variáveis preditoras e $\epsilon$ é a parte aleatória. Neste trabalho, serão usadas diferentes nomenclaturas para que os termos dos modelos não fiquem repetitivos. A variável resposta poderá ser chamada de variável dependente, evento resposta ou simplesmente resposta. As variáveis preditoras poderão ser chamadas de variáveis independentes, variáveis explicativas ou covariáveis.

\subsection{Objetivos}

Os MLGM consideram em seu preditor linear uma parte fixa e outra aleatória. Variáveis independentes podem ser introduzidas na parte fixa e/ou aleatória no preditor linear do modelo. Quando existe o interesse em considerar variáveis explicativas na parte aleatória do modelo, inclinações aleatórias para cada grupo são associadas a estas covariáveis. Na literatura, ainda não foram propostos métodos de predição para o modelo logístico misto que consideram, além do intercepto, as inclinações aleatórias.

O objetivo desta tese é propor métodos para predição da variável resposta de um modelo logístico misto considerando $k$ efeitos aleatórios, o intercepto aleatório e $k-1$ inclinações aleatórias. Quatro propostas para predição de novos grupos são apresentadas neste trabalho:

- Método de predição da média zero (MZ);

- Método de predição do melhor preditor empírico (MPE);

- Método de predição via modelos de regressão:

- regressão linear $(\mathrm{RL})$;

- modelos não-paramétricos (NP).

O MZ é o método de predição mais simples de todos, por considerar apenas a parte fixa do modelo misto na predição da resposta para novos grupos. Este método considera a média do valor dos efeitos aleatórios, que pela suposição do modelo é igual a zero.

O método MPE foi introduzido por Jiang e Lahiri (2001) no nível de grupo, e foi estendido para o nível de observação dentro do grupo por Tamura e Giampaoli (2010). Em ambos os casos o modelo misto considerou apenas um efeito aleatório. Baseado em Tamura e Giampaoli (2010), o método de predição MPE do modelo logístico misto foi estendido para $k$ efeitos aleatórios utilizando uma transformação linear multivariada e métodos computacionais para a solução das integrais $k$ dimensionais necessárias para a predição da resposta. 
Dado o esforço computacional requerido para resolver as integrais $k$-dimensionais do método MPE, foi proposta uma metodologia alternativa chamada método de predição via modelos de regressão. Esta metodologia tem o intuito de predizer os efeitos aleatórios de novos grupos considerando o efeito aleatório como variável resposta de um novo modelo de regressão. Este método utiliza um conjunto de variáveis explicativas no nível de grupo que se relacionam com o efeito aleatório através de um modelo de regressão. A metodologia de predição via modelos de regressão considera duas classes de modelos. Para o caso em que os efeitos aleatórios empíricos apresentam distribuição normal, é proposto o uso do modelo de regressão linear. Caso contrário, é proposto o uso da classe de modelos não-paramétricos.

\subsection{Contribuições}

As principais contribuições deste trabalho são as seguintes:

- Proposição de quatro novos métodos de predição da resposta de observações pertencentes a novos grupos: MZ, MPE, RL e NP;

- Comparação dos métodos de estimação propostos na literatura para o modelo logístico misto com $k$ efeitos aleatórios, por meio de estudos de simulação. Os métodos de estimação considerados foram: aproximação de Laplace, quadratura adaptativa de Gauss-Hermite com dois e cinco pontos na quadratura, e quase-verossimilhança penalizada;

- Avaliação dos métodos de predição propostos, baseados nas estimativas obtidas pelos métodos de estimação utilizados, por meio de estudos de simulação;

- Comparação do modelo logístico misto com a regressão logística tradicional em relação às metodologias abordadas;

- Discussão do custo computacional dos métodos de estimação e predição;

- Ilustração do uso dos métodos de predição para observações futuras em bancos de dados reais em distintas aplicações: uma na área da saúde pública e outra na área de marketing.

\subsection{Organização do Trabalho}

O presente trabalho encontra-se dividido em seis capítulos e um apêndice.

O primeiro capítulo, como já visto, apresenta a motivação para utilização dos modelos multiníveis e mistos, focando no modelo de regressão logística e no problema de predição de dados futuros.

O capítulo 2 revisa brevemente os principais conceitos dos modelos lineares generalizados, particularizando para o modelo linear normal e logístico. Para cada modelo, é apresentada a extensão 
para a classe dos modelos mistos com um efeito aleatório, incluindo uma revisão das contribuições na literatura em termos de predição da resposta ou do efeito aleatório. Neste capítulo também são apresentadas as definições das principais medidas de desempenho para avaliar a predição de um modelo com resposta binária.

A primeira seção do Capítulo 3 introduz o modelo logístico misto com $k$ efeitos aleatórios, e o logaritmo da função de verossimilhança marginal. Posteriormente, a segunda seção apresenta os métodos de estimação para este modelo. Logo, as duas primeiras seções deste capítulo são revisões de trabalhos presentes na literatura. A principal contribuição desta tese é apresentada na terceira seção, na qual são propostos os métodos para predição de novos grupos para o modelo logístico misto com $k$ efeitos aleatórios: média zero (MZ), melhor preditor empírico (MPE), RL (regressão linear) e NP (não-paramétrico).

Com o intuito de avaliar o desempenho da predição dos métodos propostos, foram realizados estudos de simulação, apresentados no Capítulo 4. As estimativas dos parâmetros do modelo logístico misto, considerando 2 efeitos aleatórios, foram comparados entre os métodos de estimação aproximação de Laplace, quadratura adaptativa de Gauss-Hermite e quase-verossimilhança penalizada. Os vieses das estimativas dos parâmetros também foram avaliados para verificar o quanto influenciam no desempenho dos métodos de predição e nos resíduos da predição. Para cada método de estimação, foram aplicados os métodos de predição propostos no Capítulo 3, e para cada combinação dos métodos de estimação e predição, foi avaliado o desempenho da predição da resposta usando as medidas de desempenho apresentadas no Capítulo 2 e os resíduos da predição.

No Capítulo 5, com o objetivo de ilustrar o uso de cada uma das metodologias propostas, os métodos de predição foram aplicados em dois bancos de dados reais. Uma das aplicações foi realizada na área da saúde, e a outra na área de marketing. Ambos os bancos de dados apresentaram estrutura hierárquica, cujo objetivo final foi predizer a probabilidade do evento resposta para novos grupos usando os métodos de predição propostos no Capítulo 3.

No último capítulo são apresentadas as conclusões e as sugestões de trabalhos futuros.

Finalmente, no Apêndice A podem ser visualizadas as distribuições das medidas de desempenho por cenário de simulação. 


\section{Capítulo 2}

\section{Trabalhos Relacionados}

Os trabalhos apresentados neste capítulo são importantes para o entendimento das metodologias de predição que serão propostas no Capítulo 3.

Neste capítulo, nas três primeiras seções, são revisados os principais conceitos associados a um modelo linear normal, aos modelos lineares generalizados e em especial ao modelo logístico. Para cada um dos modelos é feita a extensão para a classe dos modelos mistos.

Na quarta seção deste capítulo, são apresentadas as contribuições presentes na literatura em termos de predição para o modelo misto que considera apenas um efeito aleatório. Descreve-se em cada subseção os métodos de predição para cada classe de modelo misto.

Na última seção deste capítulo, são apresentadas algumas medidas apropriadas para avaliação de dados provenientes de uma resposta binária conhecidas na literatura como sensibilidade, especificidade, área abaixo da curva ROC e estatística KS. As medidas KS e AUC foram usadas para a avaliação das metodologias propostas em relação à predição da resposta.

\subsection{Modelo linear normal}

O melhor caminho para entender o modelo linear misto é, primeiramente, compreender o modelo linear tradicional, dado por

$$
y_{i}=\mathbf{x}_{i}^{t} \boldsymbol{\beta}+\varepsilon_{i},
$$

em que $y_{i}$ é a variável resposta da $i$-ésima observação, $\mathbf{x}_{i}^{t}$ é o vetor com dimensão $(1 \times p)$ de covariáveis conhecidas da $i$-ésima observação, $\boldsymbol{\beta}$ é o vetor com dimensão $(p \times 1)$ de coeficientes de regressão desconhecidos e $\varepsilon_{i}$ é o erro da $i$-ésima observação, para $i=1, \ldots, n$. Assume-se que $\varepsilon_{1}, \ldots, \varepsilon_{n}$ são independentes, $\operatorname{com} \varepsilon_{i} \sim \mathcal{N}\left(0, \sigma_{\varepsilon}^{2}\right)$.

No modelo (2.1), os coeficientes $\boldsymbol{\beta}$ são considerados como efeitos fixos, porém há casos em que faz sentido adicionar efeitos aleatórios. Isto ocorre tipicamente quando as observações são correlacionadas. Modelos que consideram efeitos aleatórios levam em conta a variabilidade entre os grupos, por exemplo, através da variabilidade do intercepto aleatório. Existem estudos médicos, em que a coleta da informação de um mesmo indivíduo ocorre várias vezes ao longo do tempo. Neste caso, pode-se pensar que o indivíduo representa um grupo, e assim, suas observações ao longo do tempo representam as unidades dentro do grupo. Neste exemplo, faz sentido assumir que estas 
unidades são correlacionadas, principalmente quando os intervalos de coleta ocorrem em um curto espaço de tempo. Para este tipo de problema é adequado considerar um modelo misto condicional no qual se supõe que, dado o efeito aleatório $\alpha_{i}, y_{i j}$ 's são as respostas da $j$-ésima unidade do $i$-ésimo grupo, para $j=1, \ldots, n_{i}$ e $i=1, \ldots, q$, conforme modelo dado por

$$
y_{i j} \mid \alpha_{i}=\mathbf{x}_{i j}^{t} \boldsymbol{\beta}+\alpha_{i}+\varepsilon_{i j}
$$

em que $\mathbf{x}_{i j}^{t}$ é o vetor com dimensão $(1 \times p)$ de covariáveis conhecidas, $\boldsymbol{\beta}$ é o vetor com dimensão $(p \times 1)$ de coeficientes de regressão desconhecidos, $\alpha_{i}$ é o intercepto aleatório desconhecido do $i$-ésimo grupo e $\varepsilon_{i j}$ é erro da $j$-ésima unidade do $i$-ésimo grupo. Neste caso, os $y_{i j}$ 's são condicionalmente independentes, com $y_{i j} \mid \alpha_{i} \sim \mathcal{N}\left(\mathbf{x}_{i j}^{t} \boldsymbol{\beta}+\alpha_{i}, \sigma_{\varepsilon}^{2}\right), \alpha_{1}, \ldots, \alpha_{q}$ independentes, com $\alpha_{i} \sim \mathcal{N}\left(0, \sigma^{2}\right)$, e $\varepsilon_{i j}$ 's e $\alpha_{i}$ 's independentes.

Comparando o modelo (2.1) com o modelo (2.2), fica claro que a diferença é o intercepto aleatório $\alpha_{i}$, que é um termo associado a cada grupo. Nota-se que além do intercepto aleatório, poderia-se adicionar outros coeficientes de regressão aleatórios associados à covariáveis conhecidas, estendendo assim essa classe de modelos.

Suponha agora um modelo linear misto com 2 efeitos aleatórios

$$
y_{i j} \mid\left(\alpha_{1 i}, \alpha_{2 i}\right)=\alpha_{1 i}+\alpha_{2 i} x_{i j}+\varepsilon_{i j}
$$

em que $\alpha_{1 i}$ é o intercepto aleatório, $\alpha_{2 i}$ é a inclinação aleatória associada a covariável $x_{i j}$, $\varepsilon_{i j}$ é o erro da $j$-ésima observação pertencente ao $i$-ésimo grupo. A diferença de (2.3) com o modelo linear normal (2.1), é que em (2.3) assume-se que cada grupo é caracterizado individualmente por um intercepto e uma inclinação, que são diferentes para cada grupo.

A Figura 2.1 ilustra a diferença entre o modelo tradicional e misto, em que: (a) ilustra o modelo de regressão tradicional (2.1); (b) ilustra o modelo misto (2.2) com os interceptos $\alpha_{i}$ 's, variando de grupo para grupo com o coeficiente de regressão $\beta_{i}$ constante; e (c) ilustra o modelo misto (2.3) com os interceptos $\alpha_{1 i}$ 's e os coeficientes de regressão $\alpha_{2 i}$ 's, variando de grupo para grupo.

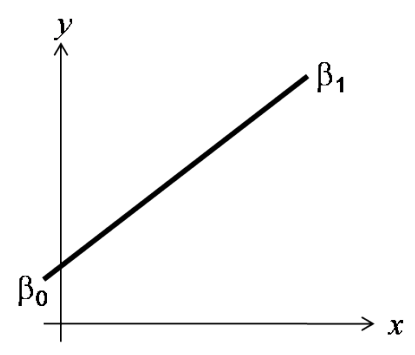

(a)

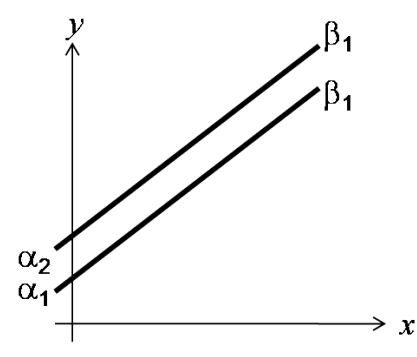

(b)

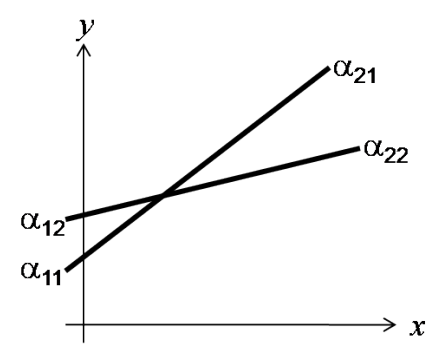

(c)

Figura 2.1: Comparação entre os modelos de regressão tradicional e o misto. 


\subsection{Modelos lineares generalizados}

Os modelos lineares têm sido usados em situações em que a variável resposta segue uma distribuição normal. Porém, há muitos casos em que a variável resposta é discreta, categorizada ou contínua sem normalidade. McCullagh e Nelder (1989) propuseram os modelos lineares generalizados (MLG) que são uma extensão dos modelos lineares. A ideia básica consiste em abrir o leque de opções para a distribuição da variável resposta que pertença à família exponencial e dar maior flexibilidade para a relação funcional entre a média da variável resposta e o preditor linear.

Sejam $y_{1}, \ldots, y_{n}$ variáveis aleatórias independentes, cada uma com densidade na forma da família exponencial dada por

$$
f\left(y_{i}\right)=\exp \left[\phi^{-1}\left\{y_{i} \theta_{i}-b\left(\theta_{i}\right)\right\}+c\left(y_{i}, \phi^{-1}\right)\right]
$$

em que $E\left(y_{i}\right)=\mu_{i}=b^{\prime}\left(\theta_{i}\right)$ e $\operatorname{Var}\left(Y_{i}\right)=\phi V_{i}, V_{i}=\frac{d \mu_{i}}{d \theta}$ é a função de variância e $\phi$ é o parâmetro de dispersão que pode ou não ser conhecido.

Os MLG são definidos por (2.4) e pela componente sistemática

$$
g\left(\mu_{i}\right)=\eta_{i}
$$

em que $\eta_{i}=\mathbf{x}_{i}^{t} \boldsymbol{\beta}$ é o preditor linear, $\boldsymbol{\beta}=\left(\beta_{1}, \ldots, \beta_{p}\right)^{t}, p<n$ é um vetor de parâmetros desconhecidos a serem estimados, o vetor $\mathbf{x}_{i}=\left(x_{i 1}, \ldots, x_{i p}\right)^{t}$ representa os valores de $p$ variáveis explicativas e $g(\cdot)$ é uma função monótona e diferenciável, denominada função de ligação.

Um caso particular importante ocorre quando o parâmetro canônico $(\theta)$ e o preditor linear $(\eta)$ coincidem, isto é, quando

$$
\theta_{i}=\eta_{i}=\sum_{c=1}^{p} x_{i c} \beta_{c}, \operatorname{com} i=1, \ldots, n,
$$

neste caso tem-se a chamada ligação canônica. Logo, obtêm-se estimativas de máxima verossimilhança únicas para os parâmetros $\beta_{1}, \ldots, \beta_{p}$, sempre que estas funções existirem, ou seja, as ligações canônicas garantem a concavidade, unicidade, existência e finitude de $L(\theta ; y)$ e, consequentemente, muitos resultados assintóticos são obtidos mais facilmente.

Os MLG cobrem uma grande variedade de modelos, entre eles, os que possuem distribuições da variável resposta Normal, Lognormal, Gama, Binomial, Poisson, Binomial Negativa, entre outras. Mais detalhes sobre os MLG podem ser encontrados, por exemplo, em Paula (2004).

No contexto de modelos mistos, a função densidade, condicional ao efeito aleatório, de um modelo linear generalizado misto (MLGM) é dada por

$$
f\left(y_{i j} \mid \alpha_{i}\right)=\exp \left[\left(\frac{a_{i j}}{\phi}\right)\left(y_{i j} \theta_{i j}-b\left(\theta_{i j}\right)\right)+c\left(y_{i j},\left(\frac{a_{i j}}{\phi}\right)\right)\right]
$$

em que $y_{i j}$ é a variável resposta da $j$-ésima observação pertencente ao $i$-ésimo grupo, para $j=$ $1, \ldots, n_{i}$ e $i=1, \ldots, q$. As quantidades $b(\cdot), c(\cdot, \cdot)$ e $\phi$ são associadas a família exponencial conforme 
descrito em (2.4) e $a_{i j}$ é um peso determinado a observação dentro do grupo.

Suponha um preditor linear que considera os efeitos fixos e o intercepto aleatório. Assim, tem-se que:

$$
g\left(\mu_{i j}\right)=\eta_{i j}=\mathbf{x}_{i j}^{t} \boldsymbol{\beta}+\alpha_{i}
$$

em que $\mathbf{x}_{i j}^{t}$ é o vetor de covariáveis conhecidas, com dimensão $(1 \times p)$, da $i$-ésima observação pertencente ao $j$-ésimo grupo, $\boldsymbol{\beta}$ é o vetor com dimensão $(p \times 1)$ de coeficientes de regressão desconhecidos e $\alpha_{i}$ é o intercepto aleatório do $i$-ésimo grupo, sendo $\alpha_{i}$ 's independentes seguindo distribuição normal com média zero e variância $\sigma^{2}$.

As funções de ligação $g(\cdot)$, canônicas para as densidades Normal, Binomial, Poisson, Gama e Normal inversa são, respectivamente, dadas por $\mu_{i j}=\eta_{i j}, \operatorname{logit}\left(\mu_{i j}\right)=\eta_{i j}, \log \left(\mu_{i j}\right)=\eta_{i j}, \mu_{i j}^{-1}=\eta_{i j}$, $\mu_{i j}^{-2}=\eta_{i j}$. Ligações não canônicas como probit e complemento log-log poderiam ser também utilizadas para o modelo Bernoulli, por exemplo. A próxima subseção trata de um caso particular dos MGLM, o modelo Bernoulli com ligação logito, conhecido como modelo logístico misto que será apresentado na próxima seção.

\subsubsection{Modelo de regressão logística}

Nesta subseção, é apresentado o modelo de regressão logística, que é um modelo particular da classe dos MLG, apropriado para variável resposta binária, ver Paula (2004).

Considere a distribuição Binomial pertencente à forma da família exponencial dada por (2.4), em que $y_{i}^{*}$ é a proporção de sucessos de $n$ ensaios independentes, cada um com probabilidade de ocorrência $\mu_{i}$. Será assumido que $n y_{i}^{*} \sim \operatorname{Bin}(n, \mu)$. A densidade de $y_{i}^{*}$ fica expressa na forma

$$
\left(\begin{array}{c}
n \\
n y_{i}^{*}
\end{array}\right) \mu_{i}^{n y_{i}^{*}}\left(1-\mu_{i}\right)^{n-n y_{i}^{*}}=\exp \left\{\log \left(\begin{array}{c}
n \\
n y_{i}^{*}
\end{array}\right)+n y_{i}^{*} \log \left(\frac{\mu_{i}}{1-\mu_{i}}\right)+n \log \left(1-\mu_{i}\right)\right\}
$$

em que $0<\mu_{i}$ e $y_{i}^{*}<1$, com $i=1, \ldots, n$. Da função densidade descrita por (2.4), para o modelo logístico $\phi=n, \theta_{i}=\log \left(\mu_{i} /\left(1-\mu_{i}\right)\right), b\left(\theta_{i}\right)=\log \left(1+\exp ^{\theta_{i}}\right), c\left(y_{i}^{*}, \phi^{-1}\right)=\log \left(\begin{array}{c}\phi \\ \phi y_{i}^{*}\end{array}\right) \mathrm{e}$ $V\left(\mu_{i}\right)=\mu_{i}\left(1-\mu_{i}\right)$.

Ao se considerar a distribuição binomial na família exponencial, tem-se que a função de ligação canônica é dada por

$$
\operatorname{logit}\left(\mu_{i}\right)=\log \left(\frac{\mu_{i}}{1-\mu_{i}}\right),
$$

resultando assim no chamado modelo logístico.

Estendendo o modelo (2.7) para a classe dos modelos mistos, o modelo logístico misto é descrito a seguir. Condicional ao intercepto aleatório $\alpha_{i}$, as respostas $y_{i j}$ 's são variáveis aleatórias independentes com distribuição Bernoulli, $\operatorname{com} i=1, \ldots, q$ e $j=1, \ldots, n_{i}$, com parâmetro $\mu_{i j}=P\left(y_{i j} \mid \alpha_{i}\right)$. 
O modelo logístico misto é dado por

$$
\operatorname{logit}\left[P\left(y_{i j}=1 \mid \alpha_{i}\right)\right]=\log \left(\frac{\mu_{i j}}{1-\mu_{i j}}\right)=\mathbf{x}_{i j}^{t} \boldsymbol{\beta}+\alpha_{i},
$$

em que $\mathbf{x}_{i j}$ é o vetor de covariáveis conhecidas, $\boldsymbol{\beta}$ é o vetor de coeficientes de regressão desconhecidos e $\alpha_{i}$ é o intercepto aleatório do $i$-ésimo grupo. Este modelo supõe que $\alpha_{1}, \alpha_{2}, \ldots, \alpha_{q}$ são i.i.d. com distribuição $\mathcal{N}\left(0, \sigma^{2}\right)$, onde $\sigma^{2}$ é a variância desconhecida.

Descritos os modelos mistos, a próxima seção apresenta os métodos de predição para novos grupos da classe dos MLGM apenas com o intercepto aleatório e, em particular, do modelo logístico misto, que o modelo considerado neste trabalho.

\subsection{Métodos de predição para modelos mistos com intercepto aleatório}

Nesta seção são apresentados os métodos de predição baseado no "Melhor Preditor" presentes na literatura. Estes métodos, que consideram apenas o intercepto aleatório, são descritos para os modelos mistos: linear normal, linear generalizado e logístico.

\subsubsection{Método de Predição MPLNVE para o modelo linear misto}

Com o objetivo de fazer a predição do efeito aleatório de um novo grupo para o modelo linear misto, o método mais conhecido é o "Melhor Preditor Linear Não Viesado" (MPLNV), em inglês BLUP ou best linear unbiased predictor.

Este assunto tem sido abordado extensivamente por uma série de autores desde os anos setenta, o que indica a importância do tema. Assim, existem muitas contribuições na literatura para o tema de predição dos efeitos aleatórios e/ou variável resposta no caso do modelo normal linear misto e multinível. A seguir são listadas algumas referências para os leitores que tiverem interesse em se aprofundar no assunto: Swamy (1970), Rosenberg (1973), Rao (1975), Harville (1976), Ware e Wu (1981), Strenio et al. (1983), Kackar e Harville (1984), Reinsel (1984), Reinsel (1985), Bondeson (1990), Candel (2004), Candel (2007), Afshartous e de Leeuw (2005) e Frees e Kim (2006).

Considere o modelo (2.2), com o intercepto aleatório $\alpha$. Sob a suposição de normalidade, o melhor preditor (MP) para $\alpha$, denotado por $\tilde{\alpha}=E(\alpha \mid y)$, é o melhor preditor linear de $\alpha$ no sentido de minimizar o erro quadrático médio $(\mathrm{EQM})$ do preditor, que é linear em $y_{i j}$. Desta forma, o preditor $\tilde{\alpha}$, chamado de MPLNV, é dado por

$$
\tilde{\alpha}_{i}=\sigma^{2}\left(\sigma^{2}+\sigma_{\varepsilon}^{2}\right)^{-1}\left(y_{i j}-\mathbf{x}_{i j}^{t} \boldsymbol{\beta}\right) .
$$

Em (2.8), $\tilde{\alpha}_{i}$ é o efeito aleatório para o $i$-ésimo novo grupo. Nota-se que esta expressão envolve 
$\boldsymbol{\beta}, \sigma^{2}$ e $\sigma_{\varepsilon}^{2}$, que na prática são desconhecidos, logo esta metodologia requer a estimativa prévia desses parâmetros. Considerando as correspondentes estimativas dos parâmetros, troca-se a notação do estimador $\tilde{\alpha}_{i}$ pela estimativa $\hat{\alpha}_{i}$, e tem-se

$$
\hat{\alpha}_{i}=\hat{\sigma}^{2}\left(\hat{\sigma}^{2}+\hat{\sigma}_{\varepsilon}^{2}\right)^{-1}\left(y_{i j}-\mathbf{x}_{i j}^{t} \hat{\boldsymbol{\beta}}\right),
$$

no qual o preditor resultante é chamado de MPLNVE (Melhor Preditor Linear Não Viesado Empírico), ou em inglês EBLUP (empirical best linear unbiased predictor). Nesta subseção, foi apresentado o caso particular do MPLNVE considerando apenas o intercepto aleatório.

Quando o modelo considera dois ou mais efeitos aleatórios, $\alpha_{i}$ e $\sigma$ são trocados por $\boldsymbol{\alpha}_{i}$ e $\boldsymbol{\Sigma}$, representando o vetor de efeitos aleatórios e a matriz de covariância dos efeitos aleatórios, respectivamente. Para mais detalhes, inclusive a obtenção de $\tilde{\alpha}_{i}$ dado em (2.8), veja Robinson (1991), que dá uma visão geral sobre o MPLNV, com exemplos e aplicações, incluindo do caso para $k$ efeitos aleatórios.

Nota-se em (2.9) que $y_{i j}$ é um valor desconhecido para a predição de novos grupos. Por esse motivo, para esta classe de modelos, os autores McCulloch e Neuhaus (2011) propuseram utilizar no lugar de $y_{i j}$ o valor $y_{i}$, que é predito a partir de um modelo linear tradicional no nível de grupo, utilizando a mesma base de dados que é realizada a estimação dos parâmetros. Portanto, a partir da equação do modelo de regressão linear tradicional e das covariáveis agredadas no nível de grupo foi possível predizer o valor do efeito aleatório de um novo grupo.

\subsubsection{Método de Predição MPE para o MLGM}

Comparado com o caso linear normal misto, há poucas contribuições para predição dos efeitos aleatórios e/ou variável resposta para a classe dos MLGM. A razão é que o resultado para predição não pode ser obtido algebricamente e expressado de forma fechada como no caso do modelo linear misto (visto na seção 2.3.1). Desta forma, os autores Jiang e Lahiri (2006), Skrondal e Rabe-Hesketh (2009) e Tamura e Giampaoli (2010) propuseram alguns métodos de predição para essa classe de modelos.

A predição pelo método de Bayes Empírico (BE), que minimiza o erro quadrático médio, é o método mais usado para atribuir valores para novos efeitos aleatórios. A razão para o termo "Bayes Empírico", que foi denotado por Robbins e Neyman (1956), é justificado pelo fato de que os princípios bayesianos são adaptados para a visão frequentista pela inserção dos parâmetros estimados do modelo no cálculo da esperança a posteriori, $E(\alpha \mid y)$. Detalhes sobre o método $\mathrm{BE}$ dos efeitos aleatórios podem ser encontrados em Efron e Morris (1973), Efron e Morris (1975), Morris (1983), Maritz e Lwin (1989), Carlin e Louis (2000a) e Carlin e Louis (2000b).

BE é conhecido também como Melhor Preditor (MP), conforme Searle et al. (1992). Por uma questão de nomenclatura, nesta tese será adotado o termo MP para referenciar esse método de predição.

Considere agora que se tem o interesse em fazer a predição do efeito aleatório ou da variável 
resposta para um MLGM. Jiang e Lahiri (2006) propuseram um método para atribuir valores do intercepto aleatório para grupos que não fizeram parte do ajuste do modelo, aplicando à classe dos MLGM a técnica do melhor preditor empírico (MPE), que será descrita a seguir.

Seja $\varsigma=\varsigma\left(\boldsymbol{\beta}, \alpha_{i}\right)$ o termo em que se tem interesse em fazer a predição, com $i=1, \ldots, q$. Seja $y_{i j}$ a variável resposta com $i$ denotando os grupos e $j$ denontando as observações dentro do grupo.

Com o objetivo de minimizar o EQM de $\tilde{\varsigma}$,

$$
\operatorname{EQM}(\tilde{\varsigma})=E(\tilde{\varsigma}-\varsigma)^{2}
$$

o MP de $\varsigma$ é dado por

$$
\tilde{\varsigma}=E(\varsigma(\boldsymbol{\beta}, \alpha) \mid y)
$$

Para facilitar o entendimento do leitor, a seguir são detalhados alguns cálculos para chegar à expressão (2.14), que foi apresentado por Jiang e Lahiri (2006). Considere que $\mathcal{S}$ é um conjunto $\operatorname{com}\{1, \ldots, q\}$, em que $i$ pertence ao conjunto $\mathcal{S}$. A esperança (2.10) pode ser escrita como

$$
\begin{gathered}
E\left(\varsigma\left(\boldsymbol{\beta}, \alpha_{\mathcal{S}}\right) \mid y_{\mathcal{S}}\right)=\int \varsigma\left(\boldsymbol{\beta}, \alpha_{\mathcal{S}}\right) f\left(\alpha_{\mathcal{S}} \mid y_{\mathcal{S}}\right) d \alpha_{\mathcal{S}}= \\
=\int \varsigma\left(\boldsymbol{\beta}, \alpha_{\mathcal{S}}\right)\left(\frac{f\left(\alpha_{\mathcal{S}}, y_{\mathcal{S}}\right)}{f\left(y_{\mathcal{S}}\right)}\right) d \alpha_{\mathcal{S}}=\int \varsigma\left(\boldsymbol{\beta}, \alpha_{\mathcal{S}}\right)\left(\frac{f\left(y_{\mathcal{S}} \mid \alpha_{\mathcal{S}}\right) f_{\alpha}\left(\alpha_{\mathcal{S}}\right)}{f\left(y_{\mathcal{S}}\right)}\right) d \alpha_{\mathcal{S}}= \\
=\frac{\int \varsigma\left(\boldsymbol{\beta}, \alpha_{\mathcal{S}}\right) f\left(y_{\mathcal{S}} \mid \alpha_{\mathcal{S}}\right) f_{\alpha}\left(\alpha_{\mathcal{S}}\right) d \alpha_{\mathcal{S}}}{f\left(y_{\mathcal{S}}\right)}
\end{gathered}
$$

Como

$$
f\left(y_{\mathcal{S}}\right)=\int f\left(y_{\mathcal{S}}, \alpha_{\mathcal{S}}\right) d \alpha_{\mathcal{S}}=\int \frac{f\left(y_{\mathcal{S}}, \alpha_{\mathcal{S}}\right)}{f_{\alpha}\left(\alpha_{\mathcal{S}}\right)} f_{\alpha}\left(\alpha_{\mathcal{S}}\right) d \alpha_{\mathcal{S}}=\int f\left(y_{\mathcal{S}} \mid \alpha_{\mathcal{S}}\right) f_{\alpha}\left(\alpha_{\mathcal{S}}\right) d \alpha_{\mathcal{S}}
$$

tem-se que

$$
\tilde{\varsigma}=\frac{\int \varsigma\left(\boldsymbol{\beta}, \alpha_{\mathcal{S}}\right) f\left(y_{\mathcal{S}} \mid \alpha_{\mathcal{S}}\right) f_{\alpha}\left(\alpha_{\mathcal{S}}\right) d \alpha_{\mathcal{S}}}{\int f\left(y_{\mathcal{S}} \mid \alpha_{\mathcal{S}}\right) f_{\alpha}\left(\alpha_{\mathcal{S}}\right) d \alpha_{\mathcal{S}}}
$$

Além disso, segundo (2.5), a função densidade condicional na forma da família exponencial é dada por

$$
f\left(y_{\mathcal{S}} \mid \alpha_{\mathcal{S}}\right)=\left\{\prod_{i \in \mathcal{S}} \prod_{j=1}^{n_{i}} f\left(y_{i j} \mid \alpha_{i}\right)\right\}=\left\{\prod_{i \in \mathcal{S}} \prod_{j=1}^{n_{i}} \exp \left[\left(\frac{a_{i j}}{\phi}\right)\left(y_{i j} \theta_{i j}-b\left(\theta_{i j}\right)\right)+c\left(y_{i j},\left(\frac{a_{i j}}{\phi}\right)\right)\right]\right\}
$$

e

$$
f_{\alpha}\left(\alpha_{\mathcal{S}}\right)=\prod_{i \in \mathcal{S}} f_{\alpha}\left(\alpha_{i}\right)
$$


Substituindo os resultados obtidos acima em (2.11), obtém-se

$$
\tilde{\varsigma}=\frac{\int \varsigma\left(\boldsymbol{\beta}, \alpha_{\mathcal{S}}\right) \exp \left(\sum_{i \in S} \sum_{j=1}^{n_{i}}\left\{\left(\frac{a_{i j}}{\phi}\right)\left(y_{i j} \theta_{i j}-b\left(\theta_{i j}\right)\right)+c\left(y_{i j}, \frac{a_{i j}}{\phi}\right)\right\}\right) \prod_{i \in \mathcal{S}} f_{\alpha}\left(\alpha_{i}\right) d \alpha_{i}}{\int \exp \left(\sum_{i \in \mathcal{S}} \sum_{j=1}^{n_{i}}\left\{\left(\frac{a_{i j}}{\phi}\right)\left(y_{i j} \theta_{i j}-b\left(\theta_{i j}\right)\right)+c\left(y_{i j}, \frac{a_{i j}}{\phi}\right)\right\}\right) \prod_{i \in \mathcal{S}} f_{\alpha}\left(\alpha_{i}\right) d \alpha_{i}} .
$$

Quando a função de ligação é canônica, $\theta_{i j}=\eta_{i j}=\mathbf{x}_{i j}^{t} \boldsymbol{\beta}+z_{i j}^{t} \alpha_{i}$. Desta forma, tem-se que

$$
\tilde{\varsigma}=\frac{\int \varsigma\left(\boldsymbol{\beta}, \alpha_{\mathcal{S}}\right) \exp \left(\sum_{i \in S} \sum_{j=1}^{n_{i}}\left\{\left(\frac{a_{i j}}{\phi}\right)\left(y_{i j}\left(\mathbf{x}_{i j}^{t} \boldsymbol{\beta}+z_{i j}^{t} \alpha_{i}\right)-b\left(\mathbf{x}_{i j}^{t} \boldsymbol{\beta}+z_{i j}^{t} \alpha_{i}\right)+c\left(y_{i j}, \frac{a_{i j}}{\phi}\right)\right\}\right) \prod_{i \in S} f_{\alpha}\left(\alpha_{i}\right) d \alpha_{i}\right.}{\left.\int \exp \left(\sum_{i \in \mathcal{S}} \sum_{j=1}^{n_{i}}\left\{\left(\frac{a_{i j}}{\phi}\right)\left(y_{i j} \mathbf{x}_{i j}^{t} \boldsymbol{\beta}+z_{i j}^{t} \alpha_{i}\right)-b\left(\mathbf{x}_{i j}^{t} \boldsymbol{\beta}+z_{i j}^{t} \alpha_{i}\right)\right)+c\left(y_{i j}, \frac{a_{i j}}{\phi}\right)\right\}\right) \prod_{i \in \mathcal{S}} f_{\alpha}\left(\alpha_{i}\right) d \alpha_{i}} .
$$

Nota-se que $\exp \left(c\left(y_{i j}, \frac{a_{i j}}{\phi}\right)\right)$ não depende de $\alpha_{i}$. Desta forma, a expressão anterior pode ser escrita como

$$
\tilde{\varsigma}=\frac{\int \varsigma\left(\boldsymbol{\beta}, \alpha_{\mathcal{S}}\right) \exp \left(\sum_{i \in \mathcal{S}} \sum_{j=1}^{n_{i}}\left\{\left(\frac{a_{i j}}{\phi}\right)\left[y_{i j}\left(\mathbf{x}_{i j}^{t} \boldsymbol{\beta}+z_{i j}^{t} \alpha_{i}\right)-b\left(\mathbf{x}_{i j}^{t} \boldsymbol{\beta}+z_{i j}^{t} \alpha_{i}\right)\right]\right\}\right) \prod_{i \in \mathcal{S}} f_{\alpha}\left(\alpha_{i}\right) d \alpha_{i}}{\int \exp \left(\sum_{i \in \mathcal{S}} \sum_{j=1}^{n_{i}}\left\{\left(\frac{a_{i j}}{\phi}\right)\left[y_{i j}\left(\mathbf{x}_{i j}^{t} \boldsymbol{\beta}+z_{i j}^{t} \alpha_{i}\right)-b\left(\mathbf{x}_{i j}^{t} \boldsymbol{\beta}+z_{i j}^{t} \alpha_{i}\right)\right]\right\}\right) \prod_{i \in \mathcal{S}} f_{\alpha}\left(\alpha_{i}\right) d \alpha_{i}} .
$$

Considere agora o caso em que o subconjunto $\mathcal{S}$ é formado apenas pelo elemento $i$, ou seja, $\mathcal{S}=\{\mathrm{i}\}$. Então, $(2.13)$ corresponde a

$$
\tilde{\varsigma}_{i}=\frac{\int \varsigma\left(\boldsymbol{\beta}, \alpha_{i}\right) \exp \left(\phi^{-1} R_{i}\left(\boldsymbol{\beta}, \alpha_{i}\right)\right) f_{\alpha}\left(\alpha_{i}\right) d \alpha_{i}}{\int \exp \left(\phi^{-1} R_{i}\left(\boldsymbol{\beta}, \alpha_{i}\right)\right) f_{\alpha}\left(\alpha_{i}\right) d \alpha_{i}}
$$

em que

$$
R_{i}\left(\boldsymbol{\beta}, \alpha_{i}\right)=\sum_{j=1}^{n_{i}} a_{i j}\left(y_{i j}\left(\mathbf{x}_{i j}^{t} \boldsymbol{\beta}+z_{i j}^{t} \alpha_{i}\right)-b\left(\mathbf{x}_{i j}^{t} \boldsymbol{\beta}+z_{i j}^{t} \alpha_{i}\right)\right)=\sum_{j=1}^{n_{i}} a_{i j}\left(y_{i j} \theta_{i j}-b\left(\theta_{i j}\right)\right)
$$

Assim,

$$
\tilde{\varsigma}_{i}=\frac{E\left(\varsigma_{i}\left(\boldsymbol{\beta}, \alpha_{i}\right) \exp \left(\phi^{-1} R_{i}\left(\boldsymbol{\beta}, \alpha_{i}\right)\right)\right)}{E\left(\exp \left(\phi^{-1} R_{i}\left(\boldsymbol{\beta}, \alpha_{i}\right)\right)\right)}
$$

Nota-se que $\tilde{\varsigma}_{i}$, o MP de $\varsigma_{i}$, depende de $y_{i}$ e $\varsigma_{i}$. Como $\varsigma_{i}$ é desconhecido, deve-se substituir $\varsigma_{i}$ por $\hat{\varsigma}_{i}$, que depende de $\left(\hat{\boldsymbol{\beta}}, \hat{\alpha}_{i}\right)$. Assim, o resultado obtido de $\hat{\varsigma}_{i}$ é denominado MPE. 


\subsubsection{Método de Predição MPE para o modelo logístico misto}

Nesta subseção são apresentados os métodos de predição do efeito aleatório (intercepto aleatório) e da variável resposta existentes na literatura para o modelo logístico misto. Jiang e Lahiri (2001) propuseram o MPE do efeito aleatório e da variável resposta no nível de grupo. Baseado em Jiang e Lahiri (2001), Tamura e Giampaoli (2010) apresentaram o MPE para predizer a variável resposta no nível de observação. As três metodologias que abordam apenas o caso com o intercepto aleatório são descritas a seguir, e alguns cálculos têm maior número de detalhes que os artigos originais para facilitar o entendimento da derivação das expressões finais.

\section{Predição do intercepto aleatório}

Suponha que se tem o interesse na estimação de $\alpha_{i}$, que representa o intercepto aleatório do $i$-ésimo grupo. Então, foi assumido que $\varsigma_{i}=\varsigma_{i}\left(\boldsymbol{\beta}, \alpha_{i}\right)=\alpha_{i}$.

Jiang e Lahiri (2001), baseado em (2.14), mostraram que o MP de $\alpha_{i}$ é dado por

$$
\tilde{\alpha}_{i}=E\left(\alpha_{i} \mid y\right)=E\left(\alpha_{i} \mid y_{i}\right)=\frac{E\left(\alpha_{i} \exp \left(\phi^{-1} R_{i}\left(\boldsymbol{\beta}, \alpha_{i}\right)\right)\right)}{E\left(\exp \left(\phi^{-1} R_{i}\left(\boldsymbol{\beta}, \alpha_{i}\right)\right)\right)}
$$

em que

$$
R_{i}\left(\boldsymbol{\beta}, \alpha_{i}\right)=\sum_{j=1}^{n_{i}}\left[y_{i j}\left(\mathbf{x}_{i j}^{t} \boldsymbol{\beta}+\alpha_{i}\right)-b\left(\mathbf{x}_{i j}^{t} \boldsymbol{\beta}+\alpha_{i}\right)\right]
$$

$\operatorname{com} a_{i j}=1$.

Substituindo $\alpha_{i}$ por $\sigma \xi$, com $\xi \sim \mathcal{N}(0,1)$ tem-se que

$$
E\left(\alpha_{i} \mid y\right)=E\left(\alpha_{i} \mid y_{i}\right)=\sigma \frac{E\left(\xi \exp \left(R_{i}(\boldsymbol{\beta}, \sigma \xi)\right)\right)}{E\left(\exp \left(R_{i}(\boldsymbol{\beta}, \sigma \xi)\right)\right)}
$$

em que

$$
R_{i}(\boldsymbol{\beta}, \sigma \xi)=\sum_{j=1}^{n_{i}}\left[\left(y_{i j} \sigma \xi\right)-\log \left(1+\exp \left(\mathbf{x}_{i j}^{t} \boldsymbol{\beta}+\sigma \xi\right)\right)\right] .
$$

O MPE de $\alpha_{i}$ é obtido substituindo $\alpha_{i}$ por $\hat{\alpha}_{i}=\varsigma_{i}(\hat{\beta}, \hat{\sigma} \xi)$. Assim, a expressão (2.15) fornece a função para predição do $i$-ésimo novo intercepto aleatório.

\section{Predição da variável resposta: nível de grupo}

Jiang e Lahiri (2001) apresentaram a função de predição da variável resposta no nível de grupo. Para derivação da função de predição e já assumindo $\alpha_{i}$ como $\sigma \xi$, considere

$$
\varsigma_{i}=\varsigma_{i}(\boldsymbol{\beta}, \sigma \xi)=p_{i}=\frac{\exp \left(\mathbf{x}_{i j}^{t} \boldsymbol{\beta}+\sigma \xi\right)}{1+\exp \left(\mathbf{x}_{i j}^{t} \boldsymbol{\beta}+\sigma \xi\right)}
$$


e também a expressão

$$
\tilde{\varsigma}_{i}=E\left(\varsigma_{i}(\boldsymbol{\beta}, \sigma \xi) \mid y_{i}\right)=p_{i}
$$

Substituindo $\sigma$ e $\boldsymbol{\beta}$ por $\hat{\sigma}$ e $\hat{\boldsymbol{\beta}}$, respectivamente, e considerando $x_{i j}=x_{i}$, em que $x_{i}$ é a informação no nível do $i$-ésimo grupo, tem-se que

$$
\hat{\varsigma}_{i}=\varsigma_{i}(\hat{\boldsymbol{\beta}}, \hat{\sigma} \xi)=p_{i}=\frac{\exp \left(\mathbf{x}_{i}^{t} \hat{\boldsymbol{\beta}}+\hat{\sigma} \xi\right)}{1+\exp \left(\mathbf{x}_{i}^{t} \hat{\boldsymbol{\beta}}+\hat{\sigma} \xi\right)} .
$$

Basedo em (2.14), o MPE é dado por

$$
\begin{gathered}
\left.\hat{\boldsymbol{\varsigma}}_{i}=p_{i}(\hat{\beta}, \hat{\sigma} \xi) \mid y_{i}\right)=\frac{E\left(\frac{\exp \left(\mathbf{x}_{i}^{t} \hat{\boldsymbol{\beta}}+\hat{\sigma} \xi\right)}{1+\exp \left(\mathbf{x}_{i}^{t} \hat{\boldsymbol{\beta}}+\hat{\sigma} \xi\right)} \exp \left(y_{i} \hat{\sigma} \xi-n_{i} \log \left(1+\exp \left(\mathbf{x}_{i}^{t} \hat{\boldsymbol{\beta}}+\hat{\sigma} \xi\right)\right)\right)\right)}{E\left(\exp \left(y_{i} \hat{\sigma} \xi-n_{i} \log \left(1+\exp \left(\mathbf{x}_{i}^{t} \hat{\boldsymbol{\beta}}+\hat{\sigma} \xi\right)\right)\right)\right)}= \\
=\frac{\exp \left(\mathbf{x}_{i}^{t} \hat{\boldsymbol{\beta}}\right) E\left(\exp \left(\left(y_{i .}+1\right) \hat{\sigma} \xi\right) \exp \left(-\left(n_{i}+1\right) \log \left(1+\exp \left(\mathbf{x}_{i}^{t} \hat{\boldsymbol{\beta}}+\hat{\sigma} \xi\right)\right)\right)\right)}{E\left(\exp \left(y_{i} \hat{\sigma} \xi-n_{i} \log \left(1+\exp \left(\mathbf{x}_{i}^{t} \hat{\boldsymbol{\beta}}+\hat{\sigma} \xi\right)\right)\right)\right)} .
\end{gathered}
$$

Logo, a probabilidade predita do $i$-ésimo novo grupo apresentar o evento de interesse para o modelo logístico misto é dada pela expressão (2.16).

\section{Predição da variável resposta: nível de unidade dentro do grupo}

Para o caso no qual se tem o interesse de fazer a predição da resposta no nível de unidade ou observação dentro do grupo, Tamura e Giampaoli (2010) estenderam o conceito de predição da resposta, baseado em Jiang e Lahiri (2001).

A função de predição da resposta no nível de observação dentro do grupo pode ser escrita como

$$
\tilde{\varsigma}_{i j}=E\left(\varsigma_{i j}(\beta, \sigma \xi) \mid y_{i j}\right)=p_{i j}\left((\beta, \sigma \xi) \mid y_{i j}\right)=\frac{\int \varsigma_{i j}(\boldsymbol{\beta}, \sigma \xi) \exp \left(R_{i}(\boldsymbol{\beta}, \sigma \xi)\right) f_{\alpha}(\sigma \xi) d \xi}{\int \exp \left(R_{i}(\boldsymbol{\beta}, \sigma \xi)\right) f_{\alpha}(\sigma \xi) d \xi}
$$

Baseado em (2.14), o MPE para predizer a variável resposta no nível de observação dentro do grupo pode ser escrita como

$$
\begin{gathered}
\hat{\varsigma}_{i j}=p_{i j}\left((\hat{\beta}, \hat{\sigma} \xi) \mid y_{i j}\right)=\frac{E\left(\frac{\exp \left(\mathbf{x}_{i j}^{t} \hat{\boldsymbol{\beta}}+\hat{\sigma} \xi\right)}{1+\exp \left(\mathbf{x}_{i j}^{t} \hat{\boldsymbol{\beta}}+\hat{\sigma} \xi\right)} \exp \left(y_{i .} \hat{\sigma} \xi-\sum_{l=1}^{n_{i}} \log \left(1+\exp \left(\mathbf{x}_{i l}^{t} \hat{\boldsymbol{\beta}}+\hat{\sigma} \xi\right)\right)\right)\right)}{E\left(\exp \left(y_{i .} \hat{\sigma} \xi-\sum_{l=1}^{n_{i}} \log \left(1+\exp \left(\mathbf{x}_{i l}^{t} \hat{\boldsymbol{\beta}}+\hat{\sigma} \xi\right)\right)\right)\right)}= \\
=\exp \left(\mathbf{x}_{i j}^{t} \hat{\boldsymbol{\beta}}\right) \frac{E\left(\frac{\exp (\hat{\sigma} \xi)}{1+\exp \left(\mathbf{x}_{i j}^{t} \hat{\boldsymbol{\beta}}+\hat{\sigma} \xi\right)} \exp \left(y_{i .} \hat{\sigma} \xi\right) \exp \left(-\sum_{l=1}^{n_{i}} \log \left(1+\exp \left(\mathbf{x}_{i l}^{t} \hat{\boldsymbol{\beta}}+\hat{\sigma} \xi\right)\right)\right)\right)}{E\left(\exp \left(y_{i .} \hat{\sigma} \xi-\sum_{l=1}^{n_{i}} \log \left(1+\exp \left(\mathbf{x}_{i l}^{t} \hat{\boldsymbol{\beta}}+\hat{\sigma} \xi\right)\right)\right)\right)} .
\end{gathered}
$$


Assim, para um modelo logístico misto, a probabilidade predita da $j$-ésima observação do $i$ ésimo novo grupo apresentar o evento de interesse é dada pela expressão (2.18).

\subsection{Medidas de desempenho para o modelo com resposta binária}

$\mathrm{Na}$ última seção deste capítulo se faz uma revisão das medidas de desempenho presentes na literatura para avaliar a predição de um modelo estatístico com evento binário.

Quando se tem o objetivo de fazer uma classificação binária baseada em uma probabilidade predita, é importante considerar medidas que indiquem a qualidade da classificação em relação à resposta observada. Dentre várias medidas existentes, foram descritas as medidas sensibilidade, especificidade, estatística Kolmogorov-Smirnov (KS) e área abaixo da curva ROC (AUC), que são medidas conhecidas e muito difundidas na literatura. Veja, por exemplo, Hosmer e Lemeshow (2000) e Fawcett (2006)

As medidas AUC e KS serão utilizadas na avaliação da qualidade de predição da variável resposta dos modelos de regressão logística nos estudos de simulação e aplicação apresentados nos próximos capítulos.

Como os métodos de predição propostos nesta tese dependem da estimação dos parâmetros, será avaliada a classificação da resposta para o ajuste do modelo, segundo o método de estimação considerado. Também será avaliada a classificação da resposta para os novos grupos, segundo os métodos de predição propostos.

Uma maneira intuitiva de sumarizar os resultados do ajuste de um modelo com resposta binária é pela tabela de classificação. Essa tabela é o resultado da classificação cruzada da resposta binária observada versus a resposta binária predita a partir de um determinado ponto de corte, conforme Tabela 2.1. Abaixo, segue a definição das caselas desta tabela:

- $V P$ (verdadeiro-positivo): quantidade de observações que o modelo classificou como positivo quando, de fato, elas são positivas;

- $V N$ (verdadeiro-negativo): quantidade de observações que o modelo classificou como negativo quando, de fato, elas são negativas;

- FP (falso-positivo): quantidade de observações que o modelo classificou como positivo quando, de fato, elas são negativas;

- FN (falso-negativo): quantidade de observações que o modelo classificou como negativo quando, de fato, elas são positivas.

As expressões nas marginais desta tabela são interpretadas por:

- $V N+F P$ : quantidade de observações que de fato são negativas;

- $F N+V P$ : quantidade de observações que de fato são positivas;

- $V N+F N$ : quantidade de observações que são classificadas como negativas; 
Tabela 2.1: Tabela de classificação - resposta observada versus resposta predita.

\begin{tabular}{|c|c|c|c|c|}
\hline \multirow{4}{*}{ Resposta Observada } & & \multicolumn{2}{|c|}{ Resposta predita } & \multirow{2}{*}{ Total } \\
\hline & & 0 & 1 & \\
\hline & 0 & VN & FP & $\mathrm{VN}+\mathrm{FP}$ \\
\hline & 1 & $\mathrm{FN}$ & VP & $\mathrm{FN}+\mathrm{VP}$ \\
\hline & Total & $\mathrm{VN}+\mathrm{FN}$ & $\mathrm{FP}+\mathrm{VP}$ & $\mathrm{VN}+\mathrm{FN}+\mathrm{FP}+\mathrm{VP}$ \\
\hline
\end{tabular}

- FP+VP: quantidade de observações que são classificadas como positivas;

- $V N+F N+F P+V P:$ quantidade total de observações.

Se o valor da probabilidade predita é maior que o ponto de corte, então se classifica a resposta predita como um e caso contrário, classifica-se como zero. Quando a variável resposta é zero, o evento é classificado como negativo e, quando vale um, como positivo. Os termos "negativo" e "positivo" são originários da área médica, no qual em testes de diagnóstico usa-se o termo "positivo" para o paciente com a presença da doença ou do evento que está sendo investigado, e o termo "negativo" é usado para a ausência do evento em questão.

A sensibilidade mede o percentual de acerto dentro do evento de interesse, ou seja, indica o quão bom é o modelo para predizer a resposta como sendo um (ou evento positivo), quando verdadeiramente a resposta observada é um. A especificidade mede o acerto dentro do evento complementar, isto é, indica o quão bom é o modelo para predizer a resposta como sendo zero (ou evento negativo), quando verdadeiramente a resposta é zero. A seguir descreve-se como são calculados esses dois índices com base na Tabela 2.1:

$$
\text { sensibilidade }=\frac{V P}{F N+V P} \quad \text { e especificidade }=\frac{V N}{V N+F P} .
$$

Mais detalhes sobre esses dois índices, ver Hosmer e Lemeshow (2000) ou Collett (2002), por exemplo.

Como a sensibilidade e a especificidade avaliam a acurácia do evento zero e um isoladamente, para a avaliação da predição do modelo logístico, optaram-se por medidas mais gerais, como AUC e KS, que consideram a combinação dos índices de sensibilidade e especificidade. Estas duas medidas, usadas comumente para avaliar a classificação da resposta binária, são revistas nas duas próximas subseções.

\subsubsection{AUC e curva ROC}

O maior problema da sensibilidade e especificidade é que essas medidas dependem de um ponto de corte, que é muitas vezes arbitrariamente definido. Assim, mudando o critério, pode-se aumentar a sensibilidade com o consequente detrimento da especificidade, ou vice-versa. A vantagem de se 
utilizar a curva ROC (Receiver Operating Characteristics) é que mesma permite a flexibilidade de considerar a sensibilidade e especificidade, porém sem depender de um determinado ponto de corte.

A análise ROC foi desenvolvida entre 1950 e 1960 para avaliar a detecção de sinais em radar e na psicologia sensorial. A potencial utilidade da análise ROC em avaliar diagnósticos médicos foi desde então vastamente utilizada e, subsequentemente, foi aplicada com sucesso a uma grande variedade de testes de diagnóstico Swets et al. (1979). Em Metz (1978), Hanley e McNeil (1982) e Fawcett (2006) são introduzidos os principais conceitos da curva ROC e do cálculo da área abaixo da curva.

Embora a curva ROC seja aparentemente simples, há alguns conceitos importantes discutidos em Fawcett (2006) que servem como conceitos introdutórios e guia para pesquisadores. A curva de ROC consiste de um gráfico bidimensional, onde o eixo horizontal é '1 - especificidade' e o eixo vertical é 'sensibilidade', que permite analisar a probabilidade de verdadeiros positivos (sensibilidade) versus os falsos positivos (1 - especificidade) para valores de corte diferentes, conforme Figura 2.2.

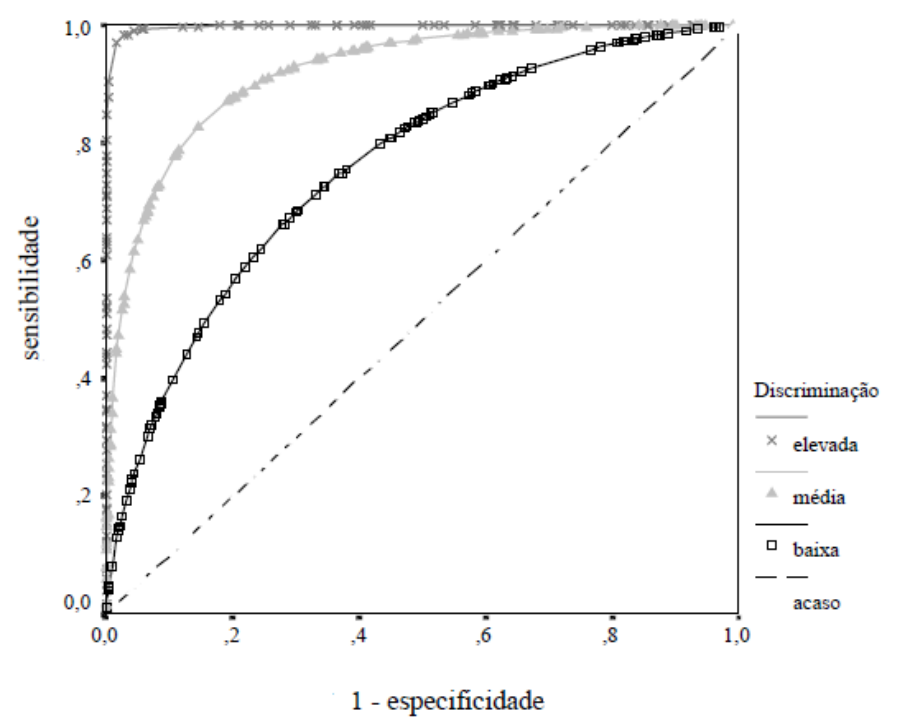

Figura 2.2: Curva ROC - figura ilustrativa.

Com base na curva ROC, a área abaixo da curva (AUC: area under the curve) é frequentemente usada como medida de desempenho. Quanto maior a área abaixo da curva, maior é a sensibilidade (verdadeiro positivo) e menor é '1-especificidade' (falso positivo). Em um modelo com classificação binária perfeita, teria-se 1 (ou 100\%) de sensibilidade e 1 (ou 100\%) de especificidade (ou equivalentemente 0 (ou 0\%) de '1-especificidade').

A Figura 2.2 mostra um exemplo ilustrativo de três curvas ROC's referentes a modelos com alta, média e baixa discriminação, sendo a linha diagonal dada pela classificação aleatória (acaso). A curva que possui maior área abaixo dela, é aquela correspondente ao modelo que apresenta melhor desempenho.

Como regra geral, utiliza-se os seguintes valores para classificar a discriminação da resposta para o índice AUC: 
- $\mathrm{AUC}=50 \%$ : discriminação aleatória,

- $50 \%<\mathrm{AUC} \leq 60 \%$ : discriminação fraca,

- $60 \%<\mathrm{AUC} \leq 70 \%$ : discriminação regular,

- $70 \%<$ AUC $\leq 80 \%$ : discriminação boa,

- $80 \%<$ AUC $\leq 90 \%$ : discriminação excelente,

- AUC > 90\%: discriminação fora do comum.

Estes valores indicam a capacidade do modelo predizer o valor da resposta para diferentes pontos de corte. Valores de AUC acima de $90 \%$ são muito raros de se encontrar na prática. Mais detalhes, veja Hosmer e Lemeshow (2000).

Neste trabalho, será utilizada como medida de desempenho o valor de AUC. A curva ROC foi necessária apenas como passo intermediário na obtenção da área abaixo dela, e por isso, a visualização gráfica não será utilizada.

\subsubsection{Estatística KS}

Para complementar a análise do modelo com resposta binária, com base na sensibilidade e especificidade, obtém-se a estatística KS que testa a hipótese de que duas populações apresentarem a mesma distribuição para uma determinada variável aleatória, conforme Conover (1999). É muito comum, na prática, principalmente em aplicações de modelos de Credit Score utilizar a estatística do teste como medida da capacidade de discriminação do modelo logístico, sendo esta medida conhecida simplesmente como KS, conforme Alves (2008). Esta estatística pode ser calculada através da combinação da sensibilidade com a especificidade da seguinte maneira:

$$
K S=\max \mid \text { sensibilidade }-(1 \text { - especificidade }) \mid
$$

sendo $\max |\cdot|$, o máximo da diferença absoluta da diferença entre a sensibilidade e a especificidade para distintos pontos de corte.

A estatística KS tem uma importante relação com a curva ROC, consequentemente com a medida AUC, pois fornece um critério para a escolha do ponto de corte ótimo para classificação do evento binário, minimizando o número total de erros de classificação. Esta medida tem uma característica atrativa, uma vez que determina o ponto da curva ROC que apresenta a maior distância em relação à classificação aleatória.

Assim como a medida AUC, o índice KS também possui uma tabela para classificar a discriminação da resposta, que na prática é usada da seguinte forma:

- $\mathrm{KS}=0 \%$ : discriminação aleatória,

- $10 \%<\mathrm{KS} \leq 20 \%$ : discriminação fraca, 
- $20 \%<\mathrm{KS} \leq 30 \%$ : discriminação regular,

- $30 \%<\mathrm{KS} \leq 40 \%$ : discriminação boa,

- $40 \%<\mathrm{KS} \leq 60 \%$ : discriminação excelente,

- $\mathrm{KS}>60 \%$ : discriminação fora do comum.

Valores de KS acima de 60\% são muito raros de se encontrar na prática.

KS é essencialmente o índice de Youden, que é expressado como

$$
\max _{c}\{\operatorname{sensibilidade}(c)+\operatorname{especificidade}(c)-1\},
$$

para um dado ponto de corte $c$, conforme Youden (1950). Apesar das expressões apresentadas serem escritas de formas diferentes, os dois índices são idênticos. Por uma questão de nomenclatura será adotado o termo KS nas análises que serão realizadas ao longo deste trabalho. 


\section{Capítulo 3}

\section{Modelo logístico misto com $k$ efeitos aleatórios: Métodos de Estimação e Predição}

Este capítulo está dividido em três seções. A primeira seção apresenta o modelo logístico com $k$ efeitos aleatórios e o logaritmo da função de verossimilhança marginal. Para estimar os parâmetros deste modelo, na segunda seção, são apresentados os métodos de estimação presentes na literatura que podem ser aplicados a este modelo. A última seção deste capítulo concentra a principal contribuição desta tese de doutorado, que apresenta as propostas de métodos de predição da resposta para novos grupos.

Para a utilização dos métodos de estimação e predição do modelo logístico misto, os dados foram separados em dois conjuntos: base de ajuste e base de predição. A base de ajuste é aquela que contém um conjunto de unidades amostrais utilizadas para estimar os parâmetros do modelo. A base de predição possui as unidades amostrais pertencentes a novos grupos, que não estavam presentes na base de ajuste. Assim, após o modelo ser ajustado por algum dos diversos métodos de estimação na base de ajuste, os métodos de predição foram aplicados na base de predição com o objetivo de realizar a predição da resposta para novos grupos.

\subsection{Modelo logístico misto}

Seja a função densidade condicional ao vetor de efeitos aleatórios $(1 \times k)$ de um MLGM, dada por

$$
f\left(y_{i j} \mid \boldsymbol{\alpha}_{i}\right)=\exp \left[\left(\frac{a_{i j}}{\phi}\right)\left(y_{i j} \theta_{i j}-b\left(\theta_{i j}\right)\right)+c\left(y_{i j},\left(\frac{\phi}{a_{i j}}\right)\right)\right],
$$

em que $y_{i j}$ é a variável resposta da $j$-ésima observação pertencente ao $i$-ésimo grupo, para $j=$ $1, \ldots, n_{i}$ e $i=1, \ldots, q ; b(\cdot), c(\cdot, \cdot)$ e $\phi$ são associados a família exponencial, conforme descrito em (2.4), e $a_{i j}$ é um peso determinado à observação dentro do grupo. Supõe-se que $\boldsymbol{\alpha}_{1}, \ldots, \boldsymbol{\alpha}_{q}$ são i.i.d. com $\boldsymbol{\alpha}_{i} \sim \mathcal{N}_{k}(\mathbf{0}, \boldsymbol{\Sigma})$, no qual $\boldsymbol{\Sigma}=\boldsymbol{\Sigma}(\boldsymbol{\kappa})$ é a matriz de covariância dos efeitos aleatórios, com $\boldsymbol{\kappa}$ sendo as componentes de variância desconhecidas da matriz $\boldsymbol{\Sigma}$.

Nota-se que a única diferença do modelo (2.5) em relação ao modelo (3.1), é que ao invés de considerar o escalar $\alpha_{i}$ representando o intercepto aleatório do $i$-ésimo grupo, é considerado o vetor $\boldsymbol{\alpha}_{i}$ que representa os $k$ efeitos aleatórios do $i$-ésimo grupo. 
O modelo logístico misto com $k$ efeitos aleatórios considera que condicional ao vetor de efeitos aleatórios $\boldsymbol{\alpha}_{i}, y_{i j}$ 's são variáveis aleatórias que seguem uma distribuição de Bernoulli com parâmetro $\mu_{i j}$, tal que

$$
P\left(y_{i j}=1 \mid \boldsymbol{\alpha}_{i}\right)=p_{i j}=\mu_{i j}
$$

no qual a esperança $E\left(y_{i j} \mid \boldsymbol{\alpha}_{i}\right)=\mu_{i j}$ é a média condicional de $y_{i j}$. O preditor linear deste modelo é definido por

$$
g\left(\mu_{i j}\right)=\operatorname{logit}\left[P\left(y_{i j}=1 \mid \boldsymbol{\alpha}_{i}\right)\right]=\log \left[\mu_{i j} /\left(1-\mu_{i j}\right)\right]=\eta_{i j}=\mathbf{x}_{i j}^{t} \boldsymbol{\beta}+\mathbf{z}_{i j}^{t} \boldsymbol{\alpha}_{i}
$$

no qual $g(\cdot)$ é a função de ligação logito, $\mathbf{x}_{i j}^{t}$ é o vetor de covariáveis conhecido $(1 \times p)$ associado ao vetor de efeitos fixos $\boldsymbol{\beta}(p \times 1)$, $\mathbf{z}_{i j}^{t}$ é o vetor de covariáveis conhecido $(1 \times k)$ associado ao vetor de efeitos aleatórios $\boldsymbol{\alpha}_{\boldsymbol{i}}(k \times 1)$ do $i$-ésimo grupo, com $i=1, \ldots, q$. O vetor $\mathbf{z}_{i j}^{t}$ é definido por $\mathbf{z}_{i j}^{t}=(1$, $\left.z_{1 i j}, z_{2 i j}, \ldots, z_{(k-1) i j}\right)$. Então, $\mathbf{z}_{i j}^{t} \boldsymbol{\alpha}_{i}=\alpha_{1 i}+\alpha_{2 i} z_{1 i j}+\ldots+\alpha_{k i} z_{(k-1) i j}$, em que $\alpha_{1 i}$ é o intercepto aleatório e $\left(\alpha_{2 i}, \ldots, \alpha_{k i}\right)$ são as inclinações aleatórias do vetor $\boldsymbol{\alpha}_{i}^{t}=\left(\alpha_{1 i}, \alpha_{2 i}, \ldots, \alpha_{k i}\right)$.

Para estimar os parâmetros de interesse do modelo (3.2), a função de verossimilhança condicional para o $i$-ésimo grupo é definida por

$$
L_{i}\left(\boldsymbol{\beta} \mid \boldsymbol{\alpha}_{i}\right)=\prod_{j=1}^{n_{i}} f\left(y_{i j} \mid \boldsymbol{\alpha}_{i}\right)=\prod_{j=1}^{n_{i}} \exp \left(\theta_{i j} y_{i j}-b\left(\theta_{i j}\right)\right)
$$

em que $f\left(. \mid\right.$.) é a função densidade condicional (3.1) de $y_{i j}$, e para o caso particular do modelo de regressão logística $\theta_{i j}=\mathbf{x}_{i j}^{t} \boldsymbol{\beta}+\mathbf{z}_{i j}^{t} \boldsymbol{\alpha}_{i}, b\left(\theta_{i j}\right)=\log \left(1+e^{\theta_{i j}}\right), \phi=1, a_{i j}=1$ e $c(.,)=$.0 . Mais detalhes em Demidenko (2004), na página 410.

A verossimilhança condicional (3.3) pode ser escrita como logaritmo da função de verossimilhança condicional, dada por

$$
l_{i}\left(y_{i j} \mid \boldsymbol{\beta}, \boldsymbol{\alpha}_{i}\right)=\sum_{j=1}^{n_{i}} \log L_{i}\left(\boldsymbol{\beta} \mid \boldsymbol{\alpha}_{i}\right)=\sum_{j=1}^{n_{i}}\left(\theta_{i j} y_{i j}-b\left(\theta_{i j}\right)\right) .
$$

O método utilizado para estimar os parâmetros $\boldsymbol{\beta}$ e as componentes de variância de $\boldsymbol{\Sigma}$ considera o logaritmo da função de verossimilhança marginal, que é expressada por

$$
l_{i}\left(y_{i j} ; \boldsymbol{\beta}, \boldsymbol{\Sigma}\right)=\int_{\alpha_{1 i}} \ldots \int_{\alpha_{k i}} l_{i}\left(y_{i j} \mid \boldsymbol{\beta},\left(\alpha_{1 i}, \ldots, \alpha_{k i}\right)\right) f_{\alpha}\left(\alpha_{1 i}, \ldots, \alpha_{k i}\right) d \alpha_{1 i} \ldots d \alpha_{k i},
$$

em que $f_{\alpha}(\cdot)$ representa a densidade normal multivariada de $\boldsymbol{\alpha}_{i}$ e $\boldsymbol{\Sigma}$ a matriz de covariância dos efeitos aleatórios $\boldsymbol{\alpha}_{i}, \operatorname{com} i=1, \ldots, q$. O logaritmo da função de verossimilhança marginal (3.5) é a integral em relação a $\boldsymbol{\alpha}_{i}=\left(\alpha_{1 i}, \ldots, \alpha_{k i}\right)$ do logaritmo da função de verossimilhança condicional (3.4) multiplicada pela distribuição multivariada dos efeitos aleatórios $\boldsymbol{\alpha}_{i}$.

Em (3.5), tem-se a verossimilhança marginal por grupo. Agora, considerando todos os grupos, 
o logaritmo da função de verossimilhança marginal pode ser escrito como

$$
\begin{gathered}
l\left(y_{i j} ; \boldsymbol{\beta}, \boldsymbol{\Sigma}\right)=\sum_{i=1}^{q} l_{i}\left(y_{i j} ; \boldsymbol{\beta}, \boldsymbol{\Sigma}\right)= \\
-\frac{q k}{2} \log (2 \pi)-\frac{q}{2} \log \boldsymbol{\Sigma}+\sum_{i=1}^{q} \log \int_{\alpha_{1 i}} \ldots \int_{\alpha_{k i}} \exp \left(\sum_{j=1}^{n_{i}}\left(\theta_{i j} y_{i j}-b\left(\theta_{i j}\right)\right)-\frac{1}{2} \boldsymbol{\alpha}_{i}^{t} \boldsymbol{\Sigma}^{-1} \boldsymbol{\alpha}_{i}\right) d \alpha_{1 i} \ldots d \alpha_{k i} .
\end{gathered}
$$

A expressão (3.6), geralmente, não pode ser avaliada analiticamente. Por esta razão, há vários métodos de estimação para resolver as questões computacionais que serão discutidas na próxima seção.

\subsection{Métodos de estimação}

Nesta seção, serão apresentados os métodos de estimação dos parâmetros de interesse do modelo logístico apresentado na seção 3.1. Os métodos de inferência discutidos terão enfoque na maximização do logaritmo da função de verossimilhança marginal (3.6), que envolve a solução de uma integral $k$ dimensional, no qual a mutidimensionalidade pode aumentar substancialmente o processamento computacional. Por esta razão, existem vários métodos de estimação que tentam resolver ou evitar tais dificuldades computacionais.

Na literatura, são propostas várias maneiras para aproximar a verossimilhança e estimar os parâmetros do modelo logístico misto, incluindo Quase-Verossimilhança Penalizada (QVP), Aproximação de Laplace (AL) e Quadratura Adaptativa de Gauss-Hermite (QAGH). Esses três métodos de estimação foram considerados neste trabalho.

Demidenko (2004) fornece uma descrição completa da quase-verossimilhança penalizada (QVP), proposta por Breslow e Clayton (1993), e AL para o resultado binário. Em Cole et al. (2003), por exemplo, estudos indicaram que AL produz estimativas menos viesadas do que QVP.

A AL é equivalente à QAGH com um ponto na quadratura, conforme Liu e Pierce (1994). Os valores de pontos de quadratura maior do que um proporcionam uma maior precisão na avaliação do logaritmo da função de verossimilhança, mas o tempo computacional é mais demorado, conforme Liu e Pierce (1994) e Rabe-Hesketh et al. (2002).

Existem também outros métodos de estimação como Monte Carlo EM (McCulloch (1997) e Booth e Hobert (1999)), Aproximação Bayesiana Zeger e Karim (1991) e o método de integração por partes Song et al. (2005). Um breve resumo sobre estes métodos de estimação podem ser vistos no livro de Jiang (2007), nas páginas 163 a 182.

\subsubsection{Aproximação de Laplace (AL)}

Quando o logaritmo da função de verossimilhança exata é difícil de ser resolvido, aproximações tornam-se alternativas naturais, e o método mais difundido para evitar a integração é a Aproximação de Laplace (AL). A ideia da AL é usar a aproximação quadrática no ponto onde o integrando atinge seu máximo. A nova função de aproximação será tratada como nova função de verossimilhança. 
A seguir é descrito como funciona o método, primeiramente para o caso unidimensional, e posteriormente para o caso multidimensional.

O método de Laplace é uma técnica desenvolvida para aproximar integrais da forma

$$
\int \exp \{-\gamma(\alpha)\} d \alpha
$$

em que $\gamma(\alpha)$ é uma função duplamente diferenciável, e $\alpha$ é uma variável unidimensional.

Pode-se expandir $\gamma(\alpha)$ em torno de $\tilde{\alpha}$ pelo Teorema de Taylor

$$
\gamma(\alpha)=\gamma(\tilde{\alpha})+\gamma^{\prime}(\tilde{\alpha})(\alpha-\tilde{\alpha})+\frac{1}{2} \gamma^{\prime \prime}(\tilde{\alpha})(\alpha-\tilde{\alpha})^{2}+R
$$

em que $\mathrm{R}$ é um resto. Mais detalhes sobre o uso do Teorema de Taylor no contexto dos métodos de estimação podem ser vistos em Tutz (2011).

Em (3.8), a função $\gamma(\cdot)$ atinge seu mínimo no ponto $\alpha=\tilde{\alpha}, \operatorname{com} \gamma^{\prime}(\tilde{\alpha})=0$ e $\gamma^{\prime \prime}(\tilde{\alpha})>0$. Sendo assim, a expansão de Taylor de segunda ordem, no ponto $\tilde{\alpha}$, é dada por

$$
\gamma(\alpha) \approx \gamma(\tilde{\alpha})+\frac{1}{2} \gamma^{\prime \prime}(\tilde{\alpha})(\alpha-\tilde{\alpha})^{2}
$$

Assim,

$$
\int \exp \{-\gamma(\alpha)\} d \alpha \approx \exp \{-\gamma(\tilde{\alpha})\} \int \exp \left\{\left(-\frac{1}{2} \gamma^{\prime \prime}(\tilde{\alpha})(\alpha-\tilde{\alpha})^{2}\right)\right\} d \alpha
$$

Sabe-se que $\int \exp \left\{-t^{2}\right\} d t=\sqrt{\pi}$, e para realizar uma substituição de variável em (3.8), denotouse

$$
-t^{2}=-\frac{1}{2} \gamma^{\prime \prime}(\tilde{\alpha})(\alpha-\tilde{\alpha})^{2} .
$$

Derivando a equação (3.11), obteve-se a igualdade

$$
\sqrt{\frac{2}{\gamma^{\prime \prime}(\tilde{\alpha})}} d t=d \alpha
$$

Assim,

$$
\int \exp \{-\gamma(\alpha)\} d \alpha \approx \sqrt{\frac{2 \pi}{\gamma^{\prime \prime}(\tilde{\alpha})}} \exp \{-\gamma(\tilde{\alpha})\}
$$

A versão multivariada de (3.12) é facilmente estendida do caso unidimensional.

Considere o vetor $k$-dimensional $\boldsymbol{\alpha}$. Agora $\gamma^{\prime}$ e $\gamma^{\prime \prime}$ denotam, respectivamente, o gradiente (vetor de primeiras derivadas) e a hessiana (matriz de segunda derivadas). Então, a versão multivariada de (3.12) pode ser escrita da seguinte forma

$$
\int \ldots \int \exp \{-\gamma(\boldsymbol{\alpha})\} d \boldsymbol{\alpha} \approx(2 \pi)^{k / 2} \gamma^{\prime \prime}(\tilde{\boldsymbol{\alpha}})^{-1 / 2} \exp \{-\gamma(\tilde{\boldsymbol{\alpha}})\}
$$


A aproximação de Laplace aproxima o integrando pela expansão de Taylor de segunda ordem no ponto $\tilde{\boldsymbol{\alpha}}$ e é exato pelo fato de $\gamma(\boldsymbol{\alpha})$ ser quadrático em $\tilde{\boldsymbol{\alpha}}$. Raudenbush et al. (2000) sugerem utilizar a expansão de Taylor até sexta ordem, e o resultado encontado pelos autores indicam que considerando esta ordem na expasão de Taylor melhora-se a aproximação. Devido à dificuldade de implementação computacional do método de AL, considerou-se neste trabalho as rotinas já existentes que considerarma a expansão de Taylor de segunda ordem.

A estimação dos parâmetros por este método será discutido na próxima subseção, uma vez que AL é um caso particular da QAGH. Além disso, a aplicação deste método na estimação por quase-verossimilhança penalizada é apresentada na subseção 3.2.3.

\subsubsection{Quadratura Adaptativa de Gauss-Hermite (QAGH)}

Primeiramente, descreve-se o método de estimação QAGH para o caso unidimensional, e posteriormente para o caso multidimensional.

Para uma função unidimensional $\gamma(\alpha)$, a regra de Gauss-Hermite aproxima integrais do tipo $s(\alpha) \exp \left\{-\alpha^{2}\right\}$ por

$$
\int s(\alpha) \exp \left\{-\alpha^{2}\right\} d \alpha \approx \sum_{e=1}^{u} s\left(\zeta_{e}\right) \omega_{e}
$$

em que o nó $\zeta_{e}$ é o e-ésimo zero do polinômio de Hermite tendo $u$ graus, e $\omega_{e}$ representam os pesos fixos que dependem de $e$. Mais detalhes sobre o polinômio de Hermite podem ser encontrados em Liu e Pierce (1994).

Uma integral frequentemente usada é baseada na densidade da normal. Quando se substitui $\alpha=\sqrt{2} \pi \sigma_{\alpha} z_{\alpha}+\mu_{\alpha}$, obtém-se

$$
\int s(\alpha) \frac{1}{\sqrt{2 \pi \sigma_{\alpha}}} \exp \left\{-\frac{\left(\alpha-\mu_{\alpha}\right)^{2}}{2 \sigma_{\alpha}^{2}}\right\} d \alpha=(\pi)^{(-1 / 2)} \int s\left(\sqrt{2} \pi \sigma_{\alpha} z_{\alpha}+\mu_{\alpha}\right) \exp \left\{-z_{\alpha}{ }^{2}\right\} d z_{\alpha},
$$

e a partir disso, a aproximação de Gauss-Hermite é dada por

$$
\int s(\alpha) f_{\mu_{\alpha}, \sigma_{\alpha}}(\alpha) \approx \sum_{e=1}^{u} s\left(\sqrt{2} \pi \sigma_{\alpha} \zeta_{e}+\mu_{\alpha}\right) v_{e}
$$

em que $v_{e}=\boldsymbol{\omega}_{e} / \sqrt{\pi}$ é o peso transformado, $v_{e}$ é o peso do $e$-ésimo zero do polinômio de Hermite e $f_{\mu_{\alpha}, \sigma_{\alpha}}(\alpha)$ é a densidade da normal (ou Gaussiana) com média $\mu_{\alpha}$ e variância $\sigma_{\alpha}$.

A quadratura adaptativa de Gauss-Hermite (QAGH) objetiva realizar uma amostragem sob uma certa região, desta forma, $\mu_{\alpha}$ e $\sigma_{\alpha}$ são escolhidas deliberadamente. Considere a integral da função $\gamma$ :

$$
\int \gamma(t) d t
$$

Por escolha, define-se $\hat{\mu}_{\alpha}=\arg \max _{\tilde{x}_{\alpha}} \gamma\left(\tilde{x}_{\alpha}\right)$ e $\hat{\sigma}_{\alpha}=\left(-\gamma^{\prime \prime}\left(\hat{\mu}_{\alpha}\right)\right)^{-1}$, e a partir de (3.16) a QAGH 
é dada por:

$$
\begin{gathered}
\int \gamma(t) d t=\int \frac{\gamma(t)}{f_{\hat{\mu}_{\alpha}, \hat{\sigma}_{\alpha}}(t)} f_{\hat{\mu}_{\alpha}, \hat{\sigma}_{\alpha}}(t) d t \\
\approx \sum_{e=1}^{u} s\left(\sqrt{2} \pi \hat{\sigma}_{\alpha} \zeta_{e}+\hat{\mu}_{\alpha}\right) v_{e}=\sqrt{2 \pi} \hat{\sigma}_{\alpha} \sum_{e=1}^{u} \exp \left\{\zeta_{e}^{2}\right\} \gamma\left(\sqrt{2} \hat{\sigma}_{\alpha} \zeta_{e}+\hat{\mu}_{\alpha}\right) v_{e},
\end{gathered}
$$

em que $s(t)=\gamma(t) / f_{\left(\hat{\mu}_{\alpha}, \hat{\sigma}_{\alpha}\right)}(t)$.

Quando é considerado somente um nó ou um ponto na quadratura, é obtido a AL pela inserção do nó e do peso do correspondente polinômio de Hermite:

$$
\int \gamma(t) d t \approx \sqrt{2 \pi} \hat{\sigma}_{\alpha} \exp \left\{\zeta^{2}\right\} \gamma\left(\sqrt{2} \hat{\sigma}_{\alpha} \zeta+\hat{\mu}_{\alpha}\right) v=\sqrt{2 \pi} \hat{\sigma}_{\alpha} \gamma\left(\hat{\mu}_{\alpha}\right)
$$

Para se fazer a relação entre AL e QAGH, nota-se que as equações (3.12) e (3.19) são equivalentes, pela associação das funções $\gamma^{\prime \prime}(\tilde{\alpha})$ e $\exp \{-\gamma(\tilde{\alpha})\}$ de (3.12) com as respectivas funções $\hat{\sigma}_{\alpha}$ e $\gamma\left(\hat{\mu}_{\alpha}\right)$ de $(3.19)$.

Desde que a AL é o caso especial de um nó na QAGH, Liu e Pierce (1994) denominaram a equação (3.16) de Aproximação de Laplace de ordem $u$, que é equivalente à nomenclatura QAGH com $u$ pontos na quadratura.

A aproximação de Gauss-Hermite para vetor $\boldsymbol{\alpha} k$-dimensional é dada por

$$
\iint \ldots \int s(\boldsymbol{\alpha}) \exp \left\{\boldsymbol{\alpha}^{t} \boldsymbol{\alpha}\right\} d \boldsymbol{\alpha} \approx \sum_{e_{1}=1}^{o_{1}} \ldots \sum_{e_{k}=1}^{o_{k}} \omega_{e_{1}}^{(1)} \ldots \omega_{e_{k}}^{(k)} s\left(\zeta_{e_{1}}^{(1)}, \ldots, \zeta_{e_{k}}^{(k)}\right),
$$

em que o nó $\zeta_{e_{m}}^{(m)}$ são os nós do e-ésimo zero do polinômio de Hermite, e $\omega_{e_{m}}^{(m)}$ representando os pesos fixos que dependem de $o_{m}, \operatorname{com} m=1, \ldots, k$. Com índice $e$ múltiplo para $\left(e_{1}, \ldots, e_{k}\right)$, $\boldsymbol{\zeta}_{\boldsymbol{e}}=\left(\zeta_{e_{1}}^{(1)}, \ldots, \zeta_{e_{k}}^{(k)}\right)$, e $\boldsymbol{\omega}_{e}=\left(\omega_{e_{1}}^{(1)} \ldots \omega_{e_{k}}^{(k)}\right)$, a expressão $(3.20)$ pode ser escrita da forma

$$
\iint \ldots \int s(\boldsymbol{\alpha}) \exp \left\{\boldsymbol{\alpha}^{t} \boldsymbol{\alpha}\right\} d \boldsymbol{\alpha} \approx \sum_{e} s\left(\boldsymbol{\zeta}_{e}\right) \boldsymbol{\omega}_{e}
$$

A QAGH para o caso $k$-dimensional é dada por

$$
\begin{gathered}
\iint \ldots \int \gamma(\mathbf{t}) d \mathbf{t} \approx \sqrt{2}(2 \pi)^{-k / 2} \sum_{e} \frac{\omega_{e_{1}}^{(1)} \ldots \omega_{e_{k}}^{(k)} \gamma\left(\sqrt{2} \boldsymbol{\Sigma}^{1 / 2} \boldsymbol{\zeta}_{e}+\boldsymbol{\mu}_{\boldsymbol{\alpha}}\right)}{|2 \pi|^{-k / 2}\left|\boldsymbol{\Sigma}_{\boldsymbol{\alpha}}\right|^{-1 / 2} \exp \left\{\boldsymbol{\zeta}_{e}^{t} \boldsymbol{\zeta}_{e}\right\}} \\
=\sqrt{2} \sum_{e}\left|\boldsymbol{\Sigma}_{\boldsymbol{\alpha}}\right|^{-1 / 2} \omega_{e_{1}}^{(1)} \ldots \omega_{e_{k}}^{(k)} \gamma\left(\sqrt{2} \boldsymbol{\Sigma}_{\boldsymbol{\alpha}}{ }^{1 / 2} \boldsymbol{\zeta}_{e}+\boldsymbol{\mu}_{\boldsymbol{\alpha}}\right) \exp \left\{\boldsymbol{\zeta}_{e}^{t} \boldsymbol{\zeta}_{e}\right\} .
\end{gathered}
$$

Mais detalhes sobre extensão do caso unidimensional para o caso $k$-dimensional podem ser vistos em Tutz (2011) na página 506.

Para fazer a estimação dos parâmetros, usa-se a função de verossimilhança marginal, que é 
função do logaritmo da função de verossimilhança marginal (3.5), escrita na forma da aproximação de Gauss-Hermite como

$$
L_{i}^{G H}(\boldsymbol{\beta}, \boldsymbol{\Sigma})=\sum_{e} \boldsymbol{\omega}_{e} f\left(y_{i j} ; \boldsymbol{\zeta}_{e},(\boldsymbol{\beta}, \boldsymbol{\Sigma})\right)
$$

em que $\boldsymbol{\zeta}_{e}$ e $\boldsymbol{\omega}_{e}$ foram definidos no início desta subseção.

A aproximação de Gauss-Hermite (3.23) é função dos parâmetros $(\boldsymbol{\beta}, \boldsymbol{\alpha})$, que provenientes do preditor linear $\mathbf{x}_{i j}^{t} \boldsymbol{\beta}+\mathbf{z}_{i j}^{t} \boldsymbol{\alpha}_{i}$ de (3.2). Usando a igualdade

$$
f^{\prime}\left(y_{i j} \mid \boldsymbol{\omega}_{e} ; \boldsymbol{\beta}, \boldsymbol{\alpha}\right)=f\left(y_{i j} ; \zeta_{e},(\boldsymbol{\beta}, \boldsymbol{\Sigma})\right)\left(\log f\left(y_{i j} ; \boldsymbol{\zeta}_{e},(\boldsymbol{\beta}, \boldsymbol{\Sigma})\right)^{\prime}\right.
$$

em que as primeiras derivadas das funções $f\left(y_{i j} ; \boldsymbol{\zeta}_{e},(\boldsymbol{\beta}, \boldsymbol{\Sigma})\right)$ e $\log f\left(y_{i j} ; \boldsymbol{\zeta}_{e},(\boldsymbol{\beta}, \boldsymbol{\Sigma})\right)$ são calculadas em relação a $(\boldsymbol{\beta}, \boldsymbol{\alpha})$, obtém-se a função escore aproximada por

$$
\sum_{e} \delta_{i e}\left(\log f\left(y_{i j} ; \boldsymbol{\zeta}_{e},(\boldsymbol{\beta}, \boldsymbol{\Sigma})\right)^{\prime}\right.
$$

em que

$$
\delta_{i e}=\frac{\boldsymbol{\omega}_{e} f\left(y_{i j} ; \boldsymbol{\zeta}_{e},(\boldsymbol{\beta}, \boldsymbol{\Sigma})\right)}{\sum_{e^{\prime}} \omega_{e^{\prime}} f\left(y_{i j} ; \boldsymbol{\zeta}_{e},(\boldsymbol{\beta}, \boldsymbol{\Sigma})\right)},
$$

$\operatorname{com} \sum_{e} \delta_{i e}=1$, e $\delta$ denotando pesos que dependem dos parâmetros $(\boldsymbol{\beta}, \boldsymbol{\alpha})$.

As estimativas de máxima verossimilhança de $(\boldsymbol{\beta}, \boldsymbol{\alpha})$ são obtidas pelo processo iterativo de $N e w$ ton Rapson ou Escore de Fisher, utilizando a função escore aproximada (3.25). Ambos os processos iterativos expandem uma determinada função em torno de um valor inicial para as estimativas de interesse, que vai sendo atualizado a cada iteração, até que um critério de parada seja satisfeito. O método iterativo de Escore de Fisher utiliza a própria função escore (3.25), e o método Escore de Fisher considera a matriz de informação de Fisher, que é a esperança de menos a segunda derivada de (3.25).

Segundo Tutz (2011), ambos os algoritmos implicam no cálculo do valor observado ou esperado da matriz de informação de Fisher. Desde que $\delta_{i e}$ de (3.26) depende dos parâmetros a serem estimados, a derivação da matriz da informação torna-se complicada. Uma alternativa seria calcular a matriz de informação observada por diferenciação numérica de (3.26). Mais detalhes em Fahrmeir et al. (1994), na seção 7.4.

\subsubsection{Quase-Verossimilhança Penalizada (QVP)}

A quase-verossimilhança é muito utilizada por causa de sua eficiência na geração dos estimadores sem a necessidade de se fazer a suposição da distribuição da variável resposta, ou seja, este método de estimação não requer a especificação de uma distribuição, somente necessita de uma especificação sobre a média e da variância.

Para um MLGM, a função a ser maximizada é a função de quase-verossimilhança modificada pela adição de um termo de penalização que depende do efeito aleatório, e por causa disso é chamada 
de quase-verossimilhança penalizada (QVP).

Sob a suposição de que as respostas são independentes, dado o vetor de efeitos aleatórios $\boldsymbol{\alpha}_{i}$, $\operatorname{com} \boldsymbol{\alpha}_{i}$ seguindo uma distribuição normal multivariada com vetor de médias igual a zero e matriz de covariância igual a $\boldsymbol{\Sigma}=\boldsymbol{\Sigma}(\boldsymbol{\kappa})$, com $\boldsymbol{\kappa}$ sendo as componentes de variância desconhecidas, a função de quase-verossimilhança $L_{Q V}$ para um MLGM usada para estimar $(\boldsymbol{\beta}, \boldsymbol{\kappa})$ é definida por

$$
L_{Q V} \propto|\boldsymbol{\Sigma}|^{-\frac{1}{2}} \iint \ldots \int \exp \left[-\frac{1}{2} \sum_{i=1}^{q} \sum_{j=1}^{n_{i}} d_{i j}-\frac{1}{2} \boldsymbol{\alpha}^{t} \boldsymbol{\Sigma}^{-1} \boldsymbol{\alpha}\right] d \boldsymbol{\alpha},
$$

em que

$$
d_{i j}=-2 \int_{y_{i j}}^{\mu_{i j}} \frac{y_{i j}-t}{a_{i j}(\phi) V(t)} d t
$$

denota a função quase-desvio. Este termo está escrito na forma da família exponencial, com $a_{i j}(\phi)=$ $\phi / \varpi_{i j}$, no qual a quantidade $\varpi_{i j}$ é um peso, e $V(\cdot)$ é uma função de variância, que é função da média $\mu_{i j}$ (mais detalhes em McCullagh e Nelder (1989)). $L_{Q V}$ de (3.27) pode ser vista como um método de inferência aproximada uma vez que não requer o conhecimento da distribuição da variável resposta $y_{i j}$. Assim, a distribuição da variável resposta será determinada quando a função de variância $V(\cdot)$ coincidir com a função de variância de alguma distribuição da família exponencial. Mais detalhes, ver Molenberghs e Verbeke (2005).

Se a variável resposta tiver distribuição normal e a função de ligação for identidade, a equação (3.27) pode ser resolvida de forma fechada. Por outro lado, se a variável resposta for discreta, a maximização desta expressão deve ser feita por procedimentos que incluam integrações numéricas a cada ciclo da iteração.

Como o enfoque deste trabalho considera uma variável resposta binária, que é o caso de uma variável discreta, a seguir serão detalhados os procedimentos para estimação dos parâmetros que incluem integração numérica.

Usando AL, dada em (3.13), o logaritmo de $L_{Q V}$, denotado por $l_{Q V}$, pode ser expressado por

$$
l_{Q V} \approx C-\frac{1}{2} \log \left|\boldsymbol{\Sigma}^{-1}\right|-\frac{1}{2} \log \left|\gamma^{\prime \prime}(\tilde{\boldsymbol{\alpha}})\right|-\gamma(\tilde{\boldsymbol{\alpha}}),
$$

em que $C$ não depende dos parâmetros, $\tilde{\boldsymbol{\alpha}}=\tilde{\boldsymbol{\alpha}}(\boldsymbol{\beta}, \boldsymbol{\kappa})$, e

$$
\gamma(\tilde{\boldsymbol{\alpha}})=-\frac{1}{2}\left(\sum_{i=1}^{q} \sum_{j=1}^{n_{i}} d_{i j}-\boldsymbol{\alpha}^{t} \boldsymbol{\Sigma}^{-1} \boldsymbol{\alpha}\right)
$$

$\operatorname{com} \tilde{\boldsymbol{\alpha}}$ minizando $\gamma(\boldsymbol{\alpha})$. Tipicamente, $\tilde{\boldsymbol{\alpha}}$ é solução da equação $\gamma^{\prime \prime}(\boldsymbol{\alpha})=0$, i.e.,

$$
\boldsymbol{\Sigma}^{-1} \boldsymbol{\alpha}-\sum_{i=1}^{q} \sum_{j=1}^{n_{i}} \frac{y_{i j}-\mu_{i j}}{a_{i j}(\phi)-V\left(\mu_{i j}\right) g^{\prime}\left(\mu_{i j}\right)} \mathbf{z}_{i j}=0
$$


em que $\mu_{i j}=\mathbf{x}_{i j}^{t} \boldsymbol{\beta}+\mathbf{z}_{i j}^{t} \boldsymbol{\alpha}_{i}$.

Diferenciando novamente em relação a $\boldsymbol{\alpha}$, tem-se que

$$
\gamma^{\prime \prime}(\boldsymbol{\alpha})=\boldsymbol{\Sigma}^{-1}+\sum_{i=1}^{q} \sum_{j=1}^{n_{i}} \frac{\mathbf{z}_{i j} \mathbf{z}_{i j}^{t}}{a_{i j}(\phi)-V\left(\mu_{i j}\right)\left[g^{\prime}\left(\mu_{i j}\right)\right]^{2}}+r,
$$

no qual o termo $r$ tem média zero. Se se troca o denominador de (3.31) por $\varpi_{i j}$, e se ignora o termo $r$, tem-se a aproximação

$$
\gamma^{\prime \prime}(\boldsymbol{\alpha}) \approx \mathbf{Z}^{t} \mathbf{W} \mathbf{Z}+\mathbf{\Sigma}^{-1}
$$

em que $\mathbf{Z}$ é a matriz de covariáveis associadas aos efeitos aleatórios, e $\mathbf{W}$ é a matriz diagonal de pesos. Combinando (3.28) com (3.32), obtém-se

$$
l_{Q} \approx C-\frac{1}{2}\left(\log \left|\mathbf{I}+\mathbf{Z}^{t} \mathbf{W} \mathbf{Z} \mathbf{\Sigma}\right|+\sum_{i=1}^{q} \sum_{j=1}^{n_{i}} \tilde{d}_{i j}-\frac{1}{2} \tilde{\boldsymbol{\alpha}}^{t} \boldsymbol{\Sigma}^{-1} \tilde{\boldsymbol{\alpha}}\right)
$$

com $\tilde{d}_{i j}$ sendo $d_{i j}$ e $\boldsymbol{\alpha}$ sendo $\tilde{\boldsymbol{\alpha}}$. Na equação (3.33), ignorando o termo $\log \left|\mathbf{I}+\mathbf{Z}^{t} \mathbf{W Z} \mathbf{\Sigma}\right|$, tem-se a função de quase-verossimilhança penalizada (QVP), dada por

$$
l_{Q V P} \approx c-\frac{1}{2}\left(\sum_{i=1}^{q} \sum_{j=1}^{n_{i}} \tilde{d}_{i j}-\frac{1}{2} \tilde{\boldsymbol{\alpha}}^{t} \boldsymbol{\Sigma}^{-1} \tilde{\boldsymbol{\alpha}}\right) .
$$

Lembrando que $\tilde{\boldsymbol{\alpha}}$ minimiza $\gamma(\boldsymbol{\alpha})$ defindo por (3.28), dado $\boldsymbol{\beta}, \tilde{\boldsymbol{\alpha}}$ o valor que maximiza a função $l_{Q V P}$. Como este depende de $\boldsymbol{\beta}$, denota-se $\tilde{\boldsymbol{\alpha}}=\tilde{\boldsymbol{\alpha}}(\boldsymbol{\beta})$. Para os elementos de $\boldsymbol{\Sigma}$ fixados, seja $\hat{\boldsymbol{\beta}}$ o valor que maximiza a $l_{Q V P}$ como uma função de $\boldsymbol{\beta}$. Então, $(\hat{\boldsymbol{\beta}}, \hat{\boldsymbol{\alpha}})=(\hat{\boldsymbol{\beta}}(\boldsymbol{\kappa}), \hat{\boldsymbol{\alpha}}(\boldsymbol{\kappa}))$, onde $\hat{\boldsymbol{\alpha}}=$ $\tilde{\boldsymbol{\alpha}}(\hat{\boldsymbol{\beta}})$, maximiza conjuntamente a QVP de Green (1987) por

$$
l_{Q V P}(\boldsymbol{\beta}, \boldsymbol{\alpha})=-\frac{1}{2} \sum_{i=1}^{q} \sum_{j=1}^{n_{i}} d_{i j}-\frac{1}{2} \boldsymbol{\alpha}^{t} \boldsymbol{\Sigma}^{-1} \boldsymbol{\alpha}
$$

como função de $\boldsymbol{\beta}$ e $\boldsymbol{\alpha}$, em que $\hat{\boldsymbol{\alpha}}=\tilde{\boldsymbol{\alpha}}(\hat{\boldsymbol{\beta}})$. Nota-se que (3.35) é o negativo de $\gamma(\boldsymbol{\alpha})$, definida em (3.28).

O método padrão para maximização de (3.35) envolve a solução de um sistema de equações não lineares, dado por

$$
\begin{gathered}
\frac{\partial l_{Q V P}(\boldsymbol{\beta}, \boldsymbol{\alpha})}{\partial \boldsymbol{\alpha}}=\sum_{i=1}^{q} \sum_{j=1}^{n_{i}} \frac{y_{i j}-\mu_{i j}}{a_{i j}(\phi)-V\left(\mu_{i j}\right) g^{\prime}\left(\mu_{i j}\right)} \mathbf{x}_{i j}=0, \\
\frac{\partial l_{Q V P}(\boldsymbol{\beta}, \boldsymbol{\alpha})}{\partial \boldsymbol{\beta}}=\sum_{i=1}^{q} \sum_{j=1}^{n_{i}} \frac{y_{i j}-\mu_{i j}}{a_{i j}(\phi)-V\left(\mu_{i j}\right) g^{\prime}\left(\mu_{i j}\right)} \mathbf{z}_{i j}-\boldsymbol{\Sigma}^{-1} \boldsymbol{\alpha}=0 .
\end{gathered}
$$

Os autores Breslow e Clayton (1993) propuseram um processo iterativo para resolver (3.36) e 
(3.37) pela modificação do algoritmo Escore de Fisher, desenvolvido por Green (1987). Uma característica atrativa do procedimento de Breslow e Clayton (1993) é que este procedimento lineariza o modelo para associá-lo ao modelo linear misto utilizando a ligação inversa, $g^{-1}(\cdot)$ e a expansão de Taylor de $1^{\mathrm{a}}$ ordem em torno dos valores $\boldsymbol{\beta}$ e $\boldsymbol{\alpha}$ inicialmente definidos para o modelo (3.2).

Primeiramente, considere $\tilde{y}_{i j}, \operatorname{com} i=1, \ldots, q$ e $j=1, \ldots, n_{i}$, com

$$
\tilde{y}_{i j}=\eta_{i j}+g^{\prime}\left(\mu_{i j}\right)\left(y_{i j}-\mu_{i j}\right)
$$

em que $\eta_{i j}$ e $\mu_{i j}$ são avaliados nos estimadores de $\boldsymbol{\beta}$ e $\boldsymbol{\alpha}$.

Então, a solução para (3.36) e (3.37) via método de Escore de Fisher podem ser expressadas como soluções iterativas do sistema

$$
\left[\begin{array}{cc}
\mathbf{X} \mathbf{T}^{\prime} \mathbf{X} & \mathbf{X} \mathbf{T}^{\prime} \mathbf{Z} \\
\mathbf{Z}^{\prime} \mathbf{T X} & \boldsymbol{\Sigma}^{-1}+\mathbf{Z}^{\prime} \mathbf{T Z}
\end{array}\right]\left[\begin{array}{l}
\boldsymbol{\beta} \\
\boldsymbol{\alpha}
\end{array}\right]=\left[\begin{array}{c}
\mathbf{X}^{\prime} \mathbf{T} \\
\mathbf{Z}^{\prime} \mathbf{T}
\end{array}\right] \tilde{\mathbf{y}}
$$

em que $\boldsymbol{\beta}$ e $\boldsymbol{\alpha}$ estão associadas ao modelo linear misto $\mathbf{Y}=\mathbf{X} \boldsymbol{\beta}+\mathbf{Z} \boldsymbol{\alpha}+\boldsymbol{\varepsilon}, \operatorname{com} \varepsilon \sim \mathcal{N}\left(0, \mathbf{T}^{-1}\right)$ e $\boldsymbol{\alpha} \sim \mathcal{N}(0, \boldsymbol{\Sigma})$, com $\boldsymbol{\varepsilon}$ e $\boldsymbol{\alpha}$ independentes. Assim, a solução do sistema (3.39) é dada por

$$
\begin{gathered}
\boldsymbol{\beta}=\left(\left(\mathbf{X}^{\prime} \mathbf{V}^{-1} \mathbf{X}\right)^{-1}\right) \mathbf{X}^{\prime} \mathbf{V}^{-1} \tilde{\mathbf{y}}, \\
\boldsymbol{\alpha}=\mathbf{\Sigma} \mathbf{Z}^{\prime} \mathbf{V}^{-1}(\tilde{y}-\mathbf{X} \boldsymbol{\beta}),
\end{gathered}
$$

em que $\mathbf{V}=\mathbf{T}^{-1}+\mathbf{X} \boldsymbol{\Sigma} \mathbf{Z}^{\prime}$, assumindo que a inversa da matriz existe, e para encontrar as estimativas de $\hat{\boldsymbol{\beta}}$ e $\hat{\boldsymbol{\alpha}}$, foi considerado que os elementos de $\boldsymbol{\Sigma}$ eram conhecidos, pois (3.40) e (3.41) dependem dos elementos $\boldsymbol{\kappa}$ de $\boldsymbol{\Sigma}$. Detalhes para a obtenção de (3.38) podem ser vistos em Tamura (2007).

Na prática, a estimação ainda envolve encontrar as estimativas das componentes de variância $\boldsymbol{\kappa}$ da matriz $\boldsymbol{\Sigma}(\boldsymbol{\kappa})$. Breslow e Clayton (1993) propuseram que substituindo o valor maximizado de (3.35) na equação (3.33), e avaliando T quando $(\hat{\boldsymbol{\beta}}(\boldsymbol{\kappa}), \hat{\boldsymbol{\alpha}}(\boldsymbol{\kappa}))$, fornece-se uma aproximação para a função de quase-verossimilhança para a inferência de $\boldsymbol{\kappa}$.

Segundo Breslow e Clayton (1993), este algoritmo produz estimativas aproximadas das soluções das equações de máxima verossimilhança restrita para os coeficientes de regressão e para os componentes da variância, no caso do modelo linear misto. Para os outros modelos este algoritmo produz estimativas aproximadas das soluções das equações de máxima verossimilhança.

A descrição completa para obtenção de $\hat{\boldsymbol{\beta}}, \hat{\boldsymbol{\alpha}}$ e $\hat{\boldsymbol{\kappa}}$ pode ser encontrada em Breslow e Clayton (1993) (nas páginas 11 e 12).

Raudenbush et al. (2000) discutem que a acurácia do método de aproximação depende de acurácia do modelo linear misto obtido a partir dos pseudo dados de $\tilde{y}_{i j}$, e justificam que a aproximação será boa quando a estatística suficiente for normalmente distribuída. Rodriguez e Goldman (1995) demostram que QVP poderia apresentar viés nas estimativas dos parâmetros quando aplicado à resposta binária. 


\subsection{Métodos de Predição}

Nesta seção, concentram-se as principais contribuições da tese de doutorado, que são as propostas para predição da resposta para o modelo logístico misto considerando $k$ efeitos aleatórios.

Como apresentado na subseção 2.3.3, as pesquisas já realizadas em termos de predição para o modelo logístico misto consideraram apenas o intercepto aleatório (1 efeito aleatório), tanto no nível de grupo quanto no nível de observação dentro do grupo. Porém, para o caso do modelo com $k$ efeitos aleatórios, ainda não existem propostas para predição no nível de observação dentro do grupo.

Desta forma, neste trabalho são propostos quatro métodos de predição diferentes:

- Método de predição da média zero (MZ);

- Método de predição do melhor preditor empírico (MPE);

- Método de predição via modelos de regressão:

- via regressão linear (RL);

- via modelos não-paramétricos (NP).

O objetivo destes métodos é predizer a probabilidade do evento de interesse de uma unidade pertencente a um novo (futuro) grupo, no qual não se conhece o valor de seus dos efeitos aleatórios.

Para o método MZ, assume-se o valor zero para os novos efeitos aleatórios, dada a suposição do modelo (2.5) em que a média conjunta dos efeitos é igual a zero.

O método MPE é a extensão da metodologia proposta por Tamura e Giampaoli (2010), no qual no lugar da integral unidimensional da equação (2.14), utilizou-se a integral $k$-dimensional, em que $k$ representa a quantidade de efeitos aleatórios presentes no modelo. Ao implementar o método MPE, foi observado que o processamento para a predição da resposta foi alto devido à complexidade computacional. Desta forma, surgiu a necessidade de estudar métodos alternativos para fazer a predição da resposta, que conseguissem manter o mesmo nível preditivo do MPE e também exigissem menos complexidade computacional na obtenção das probabilidades preditas.

Assim, foi criado um método alternativo ao MPE, denominado método via modelos de regressão. Este método consiste em prever, primeiramente, os efeitos aleatórios dos novos grupos, utilizando a relação entre as covariáveis e os efeitos aleatórios, através do modelo de regressão para cada efeito aleatório. Posteriormente, para predizer a variável resposta utilizaram-se as estimativas dos efeitos fixos do modelo logístico misto e os efeitos aleatórios preditos a partir de um outro modelo de regressão.

Neste caso, para predizer o efeito aleatório, considerou-se o efeito aleatório como variável dependente e as informações do banco de dados agregadas no nível de grupo como variáveis independentes. Como os efeitos aleatórios empíricos podem ou não seguir uma distribuição normal, utilizou-se duas classes de modelos para esta metodologia: modelo de regressão linear (Hocking (1976), Neter et al. (1996), Draper et al. (1998) ou Faraway (2002)) e modelos não-paramétricos 
(Hastie e Tibshirani (1990), Fox (2000a), Fox (2000b) ou Weisberg e Fox (2010)), conhecidos na literatura.

A seguir serão apresentados cada um dos quatro métodos detalhadamente.

\subsubsection{Método de predição da Média Zero}

O método mais simples dentre todos é o método denominado média zero (MZ). Este método está baseado na suposição do modelo (3.2), tal que $\boldsymbol{\alpha}_{1}, \ldots, \boldsymbol{\alpha}_{q}$ são i.i.d. $\operatorname{com} \boldsymbol{\alpha}_{i} \sim \mathcal{N}_{k}(\mathbf{0}, \boldsymbol{\Sigma})$.

A ideia intuitiva do método é assumir o valor da média do efeito aleatório para os novos efeitos aleatórios. No artigo de Skrondal e Rabe-Hesketh (2009), página 15, os autores fazem esta proposta para o modelo que considera apenas o intercepto aleatório, assumindo o valor zero para o intercepto aleatório dos novos grupos.

A extensão para $k$ efeitos aleatórios é simples e direta, pois o método considera o valor zero para os $k$ efeitos aleatórios dos novos grupos, e consequentemente, ignora a existência da parte aleatória do modelo e das componentes de variância de $\boldsymbol{\Sigma}$. Desta forma, para fazer a predição para observações pertencentes a novos grupos, o método MZ considera somente a parte fixa estimada pelo modelo logístico misto. Assim, a probabilidade predita da $j$-ésima unidade pertencente ao $i$-ésimo novo grupo é dada por

$$
p_{i j}=P\left(y_{i j}=1 \mid \boldsymbol{\alpha}_{i}=0\right)=\frac{\exp \left\{\mathbf{x}_{i j}^{t} \hat{\boldsymbol{\beta}}\right\}}{1+\exp \left\{\mathbf{x}_{i j}^{t} \hat{\boldsymbol{\beta}}\right\}} .
$$

Este método torna a predição do modelo misto semelhante àquela que seria realizada a partir de um modelo logístico tradicional, que considera somente a parte fixa no preditor linear. Porém, para fazer a predição para novos grupos, usa-se a estimativa dos parâmetros obtidas de um modelo que considerou a parte aleatória nas estimativas dos parâmetros fixos.

Algumas vantagens do método MZ podem ser citadas: simples implementação computacional, não requer nenhum esforço computacional para se fazer a predição para novos grupos, uma vez que a predição é feita de maneira direta utilizando a função logito, e não depende das estimativas das componentes de variância de $\boldsymbol{\Sigma}$. Por outro lado, uma desvantagem do método seria quando a parte aleatória do modelo tem "maior importância" no preditor linear $\eta_{i j}$, que neste caso é anulado. Nota-se em (3.42) que as covariáveis associadas aos efeitos aleatórios são desconsideradas, pois são multiplicadas pelo valor do $m$-ésimo efeito aleatório, que vale zero, com $m=1, \ldots, k$. 


\subsubsection{Método de predição MPE}

Considere o problema de predição baseado no Melhor Preditor Empírico (MPE) apresentado por Jiang e Lahiri (2001). O objetivo é estender a metodologia de predição da resposta para novos grupos proposta por Tamura e Giampaoli (2010), que considerava o modelo com 1 efeito aleatório, para o caso do modelo com $k$ efeitos aleatórios.

Nesta subseção, o método MPE é apresentado primeiramente para a classe dos MLGM, e posteriormente particularizado para o modelo logístico misto. Assim, apesar deste trabalho ter o enfoque no modelo logístico, a metodologia pode ser aplicada a qualquer modelo cuja distribuição da resposta possa ser escrita na forma da família exponencial.

Seja a função de predição $\tilde{\varsigma}_{i j}=\varsigma_{i j}\left(\boldsymbol{\beta}, \boldsymbol{\alpha}_{i}\right), \operatorname{com} i=1, \ldots, q$ e $j=1, \ldots, n_{i}$. O MP de $\varsigma_{i j}$ que minimiza o EQM, baseado no modelo (3.1), é definido por

$$
\tilde{\varsigma}_{i j}=E\left(\tilde{\varsigma}_{i j}\left(\boldsymbol{\beta}, \boldsymbol{\alpha}_{i}\right) \mid y_{i j}\right)
$$

O procedimento para o cálculo da esperança condicional de (3.43) é idêntico ao apresentado no Capítulo 2, das equações (2.10) até (2.14), que foi descrito em detalhes para o modelo que considerou apenas 1 efeito aleatório. Assim, (3.43) proveniente da densidade condicional (3.1) pode ser escrita como

$$
\tilde{\varsigma}_{i j}=\frac{\iint \ldots \int \varsigma_{i j}\left(\boldsymbol{\beta},\left(\alpha_{1 i}, \ldots, \alpha_{k i}\right)\right) \exp \left(\phi^{-1} R_{i}\left(\boldsymbol{\beta},\left(\alpha_{1 i}, \ldots, \alpha_{k i}\right)\right)\right) f_{\alpha}\left(\alpha_{1 i}, \ldots, \alpha_{k i}\right) d \alpha_{1 i} \ldots d \alpha_{k i}}{\iint \ldots \int \exp \left(\phi^{-1} R_{i}\left(\boldsymbol{\beta},\left(\alpha_{1 i}, \ldots, \alpha_{k i}\right)\right)\right) f_{\alpha}\left(\alpha_{1 i}, \ldots, \alpha_{k i}\right) d \alpha_{1 i} \ldots d \alpha_{k i}}
$$

em que

$$
R_{i}\left(\boldsymbol{\beta}, \boldsymbol{\alpha}_{i}\right)=\sum_{j=1}^{n_{i}} a_{i j}\left(y_{i j}\left(\mathbf{x}_{i j}^{t} \boldsymbol{\beta}+\mathbf{z}_{i j}^{t} \boldsymbol{\alpha}_{i}\right)-b\left(\mathbf{x}_{i j}^{t} \boldsymbol{\beta}+\mathbf{z}_{i j}^{t} \boldsymbol{\alpha}_{i}\right)\right)=\sum_{j=1}^{n_{i}} a_{i j}\left(y_{i j} \theta_{i j}-b\left(\theta_{i j}\right)\right)
$$

e $f_{\alpha}(\cdot)$ é a densidade normal multivariada.

Equivalentemente, a expressão (2.14) pode ser escrita como

$$
\tilde{\varsigma}_{i j}=\frac{E\left(\varsigma_{i j}\left(\boldsymbol{\beta},\left(\alpha_{1 i}, \ldots, \alpha_{k i}\right)\right) \exp \left(\phi^{-1} R_{i}\left(\boldsymbol{\beta},\left(\alpha_{1 i}, \ldots, \alpha_{k i}\right)\right)\right)\right)}{E\left(\exp \left(\phi^{-1} R_{i}\left(\boldsymbol{\beta},\left(\alpha_{1 i}, \ldots, \alpha_{k i}\right)\right)\right)\right)}
$$

Em (3.44), uma transformação linear multivariada para o $i$-ésimo grupo foi necessária para transformar $f_{\alpha}(\cdot)$ em uma densidade normal padrão multivariada, conforme descrito a seguir.

Considere a transformação linear multivariada

$$
\boldsymbol{\alpha}_{i}=\boldsymbol{\Sigma}^{1 / 2} \boldsymbol{\xi}_{\boldsymbol{i}}
$$


em que $\boldsymbol{\Sigma}^{1 / 2}$ é a raiz quadrada da matriz de covariância (simétrica) dada por

$$
\boldsymbol{\Sigma}^{1 / 2}=\left(\begin{array}{cccc}
v_{11} & v_{12} & \cdots & v_{1 k} \\
v_{21} & v_{22} & \cdots & v_{2 k} \\
\vdots & \vdots & \ddots & \vdots \\
v_{k 1} & v_{k 2} & \cdots & v_{k k}
\end{array}\right)
$$

Em Timm (2002), no Capítulo 2, é apresentado como realizar o cálculo da raiz quadrada de uma matriz simética baseado na decomposição espectral.

A densidade conjunta normal multivariada de $\boldsymbol{\alpha}_{i}$ pode ser escrita como

$$
f_{\alpha}\left(\boldsymbol{\alpha}_{i}\right)=\frac{1}{(\sqrt{2 \pi})^{k}|\boldsymbol{\Sigma}|^{1 / 2}} e^{-\boldsymbol{\alpha}_{i}^{t} \Sigma^{-1} \boldsymbol{\alpha}_{i}} .
$$

A expressão para a transformação linear multivariada baseada em (3.45) é

$$
g_{\alpha}\left(\boldsymbol{\xi}_{i}\right)=f_{\alpha}\left(\boldsymbol{\alpha}_{i}\right) \operatorname{abs}|\boldsymbol{\Sigma}|^{1 / 2}
$$

em que abs $\left|\boldsymbol{\Sigma}^{\mathbf{1 / 2}}\right|$ representa o valor absoluto do determinante de $|\boldsymbol{\Sigma}|^{1 / 2}$, que é o Jacobiano da transformação. Como $\boldsymbol{\Sigma}$ é uma matriz positiva definida,

$$
\begin{gathered}
g_{\alpha}\left(\boldsymbol{\xi}_{i}\right)=f_{\alpha}\left(\boldsymbol{\alpha}_{i}\right) \text { abs }\left|\boldsymbol{\Sigma}^{1 / 2}\right|=f_{\alpha}\left(\boldsymbol{\alpha}_{i}\right)|\boldsymbol{\Sigma}|^{1 / 2} \\
=\frac{1}{(\sqrt{2 \pi})^{k}|\boldsymbol{\Sigma}|^{1 / 2}} e^{-\boldsymbol{\alpha}_{i}^{t} \Sigma^{-1} \boldsymbol{\alpha}_{i}}|\boldsymbol{\Sigma}|^{1 / 2}=\frac{1}{(\sqrt{2 \pi})^{k}} e^{-\boldsymbol{\alpha}_{i}^{t} \Sigma^{-1} \boldsymbol{\alpha}_{i}} \\
=\frac{1}{(\sqrt{2 \pi})^{k}} e^{-\left(\boldsymbol{\Sigma}^{\mathbf{1} / \mathbf{2}} \boldsymbol{\xi}_{i}\right)^{t} \boldsymbol{\Sigma}^{-1}\left(\boldsymbol{\Sigma}^{\mathbf{1} / 2} \boldsymbol{\xi}_{i}\right)}=\frac{1}{(\sqrt{2 \pi})^{k}} e^{-\boldsymbol{\xi}_{i}{ }^{t} \boldsymbol{\Sigma}^{1 / 2} \boldsymbol{\Sigma}^{-1} \boldsymbol{\Sigma}^{\mathbf{1} / \mathbf{2}} \boldsymbol{\xi}_{\boldsymbol{i}}} \\
=\frac{1}{(\sqrt{2 \pi})^{k}} e^{-\boldsymbol{\xi}_{i}{ }^{t} \boldsymbol{I} \boldsymbol{\xi}_{\boldsymbol{i}},}
\end{gathered}
$$

ou seja, $\boldsymbol{\xi}_{\boldsymbol{i}} \sim \mathcal{N}_{k}(\mathbf{0}, \boldsymbol{I})$. Mais detalhes da transformação, podem ser visto em Rencher (1998).

Então, a transformação linear (3.45) pode ser escrita como

$$
\boldsymbol{\Sigma}^{1 / 2} \xi=\left(\begin{array}{cccc}
v_{11} & v_{12} & \cdots & v_{1 k} \\
v_{21} & v_{22} & \cdots & v_{2 k} \\
\vdots & \vdots & \ddots & \vdots \\
v_{k 1} & v_{k 2} & \cdots & v_{k k}
\end{array}\right)\left(\begin{array}{c}
\xi_{1} \\
\xi_{2} \\
\vdots \\
\xi_{k}
\end{array}\right)=\left(\begin{array}{c}
\xi_{1} v_{11}+\xi_{2} v_{12}+\cdots+\xi_{k} v_{1 k} \\
\xi_{1} v_{21}+\xi_{2} v_{22}+\cdots+\xi_{k} v_{2 k} \\
\vdots \\
\xi_{1} v_{k 1}+\xi_{2} v_{k 2}+\cdots+\xi_{k} v_{k k}
\end{array}\right)
$$

Como não se conhece o valor do efeito aleatório de um novo grupo, utilizou-se a informação proveniente da distribuição de $\boldsymbol{\alpha}_{i} \sim \mathcal{N}_{k}(\mathbf{0}, \boldsymbol{\Sigma})$, que é dada pela suposição do modelo (3.1). Então, para a predição de novos grupos, considerou-se os elementos da matriz de covariância e a distribuição 
de $\boldsymbol{\xi}$, e alterou-se a notação, chamando $\boldsymbol{\alpha}_{\boldsymbol{i}}=\boldsymbol{u}$. Assim,

$$
\left(\begin{array}{c}
u_{1} \\
u_{2} \\
\vdots \\
u_{k}
\end{array}\right)=\left(\begin{array}{c}
\xi_{1} v_{11}+\xi_{2} v_{12}+\cdots+\xi_{k} v_{1 k} \\
\xi_{1} v_{21}+\xi_{2} v_{22}+\cdots+\xi_{k} v_{2 k} \\
\vdots \\
\xi_{1} v_{k 1}+\xi_{2} v_{k 2}+\cdots+\xi_{k} v_{k k}
\end{array}\right)
$$

Desta forma, a função de predição para a $j$-ésima observação dentro do $i$-ésimo novo grupo, dada por (3.44), pode ser escrita como

$$
\tilde{\varsigma}_{i j}\left(\boldsymbol{\beta}, \boldsymbol{\Sigma}^{\mathbf{1 / 2}} \boldsymbol{\xi}\right)=\frac{\int_{\xi_{1}} \ldots \int_{\xi k}\left(\varsigma_{i j}\left(\boldsymbol{\beta},\left(u_{1}, \ldots, u_{k}\right)\right) \cdot \exp \left(\phi^{-1} R_{i}\left(\boldsymbol{\beta},\left(u_{1}, \ldots, u_{k}\right)\right)\right)\right) g_{\alpha}\left(\xi_{1}, \ldots, \xi_{k}\right) d \xi_{1} \ldots d \xi_{k}}{\int_{\xi_{1}} \ldots \int_{\xi_{k}} \exp \left(\phi^{-1} R_{i}\left(\boldsymbol{\beta},\left(u_{1}, \ldots, u_{k}\right)\right)\right) f_{\alpha}\left(\xi_{1}, \ldots, \xi_{k}\right) d \xi_{1} \ldots d \xi_{k}},
$$

em que $g_{\alpha}\left(\xi_{1}, \ldots, \xi_{k}\right)=g_{\alpha}\left(\xi_{1}\right) \cdots g_{\alpha}\left(\xi_{k}\right)$ e $g_{\alpha}\left(\xi_{m}\right)$ é a densidade normal padrão univariada com $m=1, \ldots, k$.

Nota-se que a expressão apresentada por (3.46) pode ser aplicada para qualquer modelo pertencente à classe dos MLGM.

O objetivo agora é detalhar a equação (3.46) para a distribuição Bernoulli com função de ligação logito. Neste caso, seja a função de predição a variável resposta dada por

$$
\varsigma_{i j}\left(\boldsymbol{\beta}, \boldsymbol{\alpha}_{i}\right)=p_{i j}
$$

em que

$$
\begin{gathered}
p_{i j}=\frac{\exp \left\{\mathbf{x}_{i j}^{t} \boldsymbol{\beta}+\mathbf{z}_{i j} \boldsymbol{\alpha}_{i}\right\}}{1+\exp \left\{\mathbf{x}_{i j}^{t} \boldsymbol{\beta}+\mathbf{z}_{i j} \boldsymbol{\alpha}_{i}\right\}} \\
=\frac{\exp \left\{\mathbf{x}_{i j}^{t} \boldsymbol{\beta}+\alpha_{1 i}+\alpha_{2 i} z_{1 i j}+\ldots+\alpha_{k i} z_{(k-1) i j}\right\}}{1+\exp \left\{\mathbf{x}_{i j}^{t} \boldsymbol{\beta}+\alpha_{1 i}+\alpha_{2 i} z_{1 i j}+\ldots+\alpha_{k i} z_{(k-1) i j}\right\}}
\end{gathered}
$$

Seja

$$
R_{i}\left(\boldsymbol{\beta}, \boldsymbol{\alpha}_{i}\right)=\sum_{j=1}^{n_{i}}\left[y_{i j}\left(\mathbf{x}_{i j}^{t} \boldsymbol{\beta}+\alpha_{1 i}+\ldots+\alpha_{k i} z_{(k-1) i j}\right)-\log \left(1+\exp \left(\mathbf{x}_{i j}^{t} \boldsymbol{\beta}+\alpha_{1 i}+\ldots+\alpha_{k i} z_{(k-1) i j}\right)\right)\right],
$$

com $a_{i j}=1$, representando mesmo peso para todas as observações.

Foram substituídos $\boldsymbol{\Sigma}^{1 / 2}, \boldsymbol{\beta}$ e $\mathbf{x}_{i j}^{t} \boldsymbol{\beta}+\mathbf{z}_{i j}^{t} \boldsymbol{\alpha}_{i}$ por $\hat{\boldsymbol{\Sigma}}^{1 / 2}, \hat{\boldsymbol{\beta}}$ e $\mathbf{x}_{i j}^{t} \hat{\boldsymbol{\beta}}+\mathbf{z}_{i j}^{t} \hat{\boldsymbol{u}}=\mathbf{x}_{i j}^{t} \hat{\boldsymbol{\beta}}+\left(\xi_{1} \hat{v}_{11}+\ldots+\right.$ $\left.\xi_{k} \hat{v}_{1 k}\right)+\ldots+\left(\xi_{1} \hat{v}_{k 1}+\ldots+\xi_{k} \hat{v}_{k k}\right) z_{(k-1) i j}$, respectivamente. Então, o MPE de $p_{i j}$ para o modelo logístico misto é dado por

$$
\hat{p}_{i j}\left(\hat{\boldsymbol{\beta}}, \hat{\boldsymbol{\Sigma}}^{\mathbf{1} / \mathbf{2}} \boldsymbol{\xi}\right)=\frac{E\left(\frac{\exp \left(\mathbf{x}_{i j}^{t} \hat{\boldsymbol{\beta}}+\mathbf{z}_{i j}^{t} \hat{\boldsymbol{u}}\right)}{1+\exp \left(\mathbf{x}_{i j}^{t} \hat{\boldsymbol{\beta}}+\mathbf{z}_{i j}^{t} \hat{\boldsymbol{u}}\right)} \exp \left(y_{i .}\left(\mathbf{x}_{i j}^{t} \hat{\boldsymbol{\beta}}+\mathbf{z}_{i j}^{t} \hat{\boldsymbol{u}}\right)-\sum_{l=1}^{n_{i}} \log \left(1+\exp \left(\mathbf{x}_{i l}^{t} \hat{\boldsymbol{\beta}}+\mathbf{z}_{i l}^{t} \hat{\boldsymbol{u}}\right)\right)\right)\right)}{E\left(\exp \left(y_{i .}\left(\mathbf{x}_{i j}^{t} \hat{\boldsymbol{\beta}}+\mathbf{z}_{i j}^{t} \hat{\boldsymbol{u}}\right)-\sum_{l=1}^{n_{i}} \log \left(1+\exp \left(\mathbf{x}_{i l}^{t} \hat{\boldsymbol{\beta}}+\mathbf{z}_{i l}^{t} \hat{\boldsymbol{u}}\right)\right)\right)\right)}
$$




$$
\begin{gathered}
=\frac{\exp \left(\mathbf{x}_{i j}^{t} \hat{\boldsymbol{\beta}}\right) E\left(\frac{\exp \left(\left(y_{i .}+1\right) \mathbf{z}_{i j}^{t} \hat{\boldsymbol{u}}\right)}{1+\exp \left(\mathbf{x}_{i j}^{t} \hat{\boldsymbol{\beta}}+\mathbf{z}_{i j}^{t} \hat{\boldsymbol{u}}\right)} \exp \left(-\sum_{l=1}^{n_{i}} \log \left(1+\exp \left(\mathbf{x}_{i l}^{t} \hat{\boldsymbol{\beta}}+\mathbf{z}_{i l}^{t} \hat{\boldsymbol{u}}\right)\right)\right)\right.}{E\left(\exp \left(y_{i .} \mathbf{z}_{i j}^{t} \hat{\boldsymbol{u}}\right) \exp \left(-\sum_{l=1}^{n_{i}} \log \left(1+\exp \left(\mathbf{x}_{i l}^{t} \hat{\boldsymbol{\beta}}+\mathbf{z}_{i l}^{t} \hat{\boldsymbol{u}}\right)\right)\right)\right)} \\
=\frac{\left.\left.\exp \left(\mathbf{x}_{i j}^{t} \hat{\boldsymbol{\beta}}\right) E\left(\frac{\exp \left(\left(y_{i .}+1\right) \mathbf{z}_{i j}^{t} \hat{\boldsymbol{u}}\right)}{1+\exp \left(\mathbf{x}_{i j}^{t} \hat{\boldsymbol{\beta}}+\mathbf{z}_{i j}^{t} \hat{\boldsymbol{u}}\right)} \prod_{l=1}^{n_{i}} \frac{1}{1+\exp \left(\mathbf{x}_{i l}^{t} \hat{\boldsymbol{\beta}}+\mathbf{z}_{i l}^{t} \hat{\boldsymbol{u}}\right)}\right)\right)\right)}{\left.E\left(\exp \left(y_{i .} \mathbf{z}_{i j}^{t} \hat{\boldsymbol{u}}\right) \prod_{l=1}^{n_{i}} \frac{1}{1+\exp \left(\mathbf{x}_{i l}^{t} \hat{\boldsymbol{\beta}}+\mathbf{z}_{i l}^{t} \hat{\boldsymbol{u}}\right)}\right)\right)},
\end{gathered}
$$

no qual as esperanças são calculadas em relação a $\boldsymbol{\xi} \sim \mathcal{N}_{k}(\mathbf{0}, \boldsymbol{I})$.

Equivalentemente, o MPE para a $j$-ésima observação dentro do $i$-ésimo novo grupo pode ser escrito como

$$
\hat{p}_{i j}\left(\hat{\boldsymbol{\beta}}, \hat{\boldsymbol{\Sigma}}^{\mathbf{1 / 2}} \boldsymbol{\xi}\right)=\frac{\exp \left(\mathbf{x}_{i j}^{t} \hat{\boldsymbol{\beta}}\right) \int_{\xi_{1}} \ldots \int_{\xi k} \frac{\exp \left(\left(y_{i .}+1\right) \mathbf{z}_{i j}^{t} \hat{\boldsymbol{u}}\right)}{1+\exp \left(\mathbf{x}_{i j}^{t} \hat{\boldsymbol{\beta}}+\mathbf{z}_{i j}^{t} \hat{\boldsymbol{u}}\right)} \prod_{l=1}^{n_{i}} \frac{1}{1+\exp \left(\mathbf{x}_{i l}^{t} \hat{\boldsymbol{\beta}}+\mathbf{z}_{i l}^{t} \hat{\boldsymbol{u}}\right)} g_{\alpha}\left(\xi_{1}, \ldots, \xi_{k}\right) d \xi_{1} \ldots d \xi_{k}}{\int_{\xi_{1}} \ldots \int_{\xi_{k}} \exp \left(y_{i .} \mathbf{z}_{i j}^{t} \hat{\boldsymbol{u}}\right) \prod_{l=1}^{n_{i}} \frac{1}{1+\exp \left(\mathbf{x}_{i l}^{t} \hat{\boldsymbol{\beta}}+\mathbf{z}_{i l}^{t} \hat{\boldsymbol{u}}\right)} g_{\alpha}\left(\xi_{1}, \ldots, \xi_{k}\right) d \xi_{1} \ldots d \xi_{k}},
$$

em que $g_{\alpha}\left(\xi_{1}, \ldots, \xi_{k}\right)=g_{\alpha}\left(\xi_{1}\right) \cdots g_{\alpha}\left(\xi_{k}\right)$, e $g_{\alpha}\left(\xi_{m}\right)$ é a densidade da normal padrão univariada com $m=1, \ldots, k$.

Nota-se que em (3.48) que para predição de novos grupos não se conhece o valor de $y_{i}$. Na literatura são apresentadas algumas formas de atribuir um valor para $y_{i}$. Tamura e Giampaoli (2010) assumiram $y_{i .}=n_{i .} / 2$ representando igual probabilidade de sucesso e falha para o evento de interesse para cada observação dentro de um determinado grupo. Essa suposição faz sentido, uma vez que quando não se conhece a resposta para novos grupos, e assume-se que a mesma poderia ocorrer para metade das observações dentro do grupo.

Outra forma de atribuir um valor para $y_{i}$, seria predizer a probabilidade de sucesso para novo grupo, gerada por um modelo de regressão logística ajustado previamente no nível de grupo, e posteriormente multiplicado pela quantidade de observações dentro do grupo, i.e.,

$$
y_{i .}=p_{i .} n_{i .}=\frac{\exp \left(\mathbf{w}_{i}^{t} \boldsymbol{\lambda}\right)}{1+\exp \left(\mathbf{w}_{i}^{t} \boldsymbol{\lambda}\right)} n_{i .}
$$

com $\mathbf{w}_{i}^{t}=\left(\mathbf{x}_{i}^{t}, \mathbf{z}_{i}^{t}\right)$ sendo as covariáveis agregadas no nível de grupo, $\boldsymbol{\lambda}$ os coeficientes de regressão associados às covariáveis $\mathbf{w}_{i}$ e $n_{i}$. a quantidade de observações dentro do grupo. A ideia de aproximar a resposta média do grupo com base em um outro modelo ajustado no nível de grupo é similar àquela adotada por McCulloch e Neuhaus (2011) que obtiveram $y_{i}$. para um modelo linear misto com resposta normal. Neste trabalho foram consideradas ambas as abordagens para "aproximar"o valor de $y_{i}$ que é desconhecido para novos grupos.

Como o método envolve uma complexa implementação computacional que depende de rotinas 
de programação específicas, o código para implementação do MPE para o modelo logístico com dois efeitos aleatórios (o intercepto aleatório e uma inclinação aleatória) está disponível por meio de contato com o autor, pelo e-mail: karin.ayumi.tamura@gmail.com. A implementação para mais de dois efeitos aleatórios pode ser desenvolvida utilizando as mesmas rotinas presentes no código de implementação.

\subsubsection{Método de predição via modelos de regressão}

Reiterando que o método MPE exige complexidade computacional para o cálculo das integrais multidimensionais, foi estudada outra metodologia para predição dos valores dos efeitos aleatórios de novos grupos.

O objetivo do método é modelar a dependência da resposta, o efeito aleatório $\left(\alpha_{m}\right)$, em relação às covariáveis agregadas no nível de grupo. Então, baseado nas covariáveis, é possível predizer os efeitos aleatórios para os novos grupos, que por sua vez são incluídos dentro da função logito do modelo logístico misto.

Esta metodologia, denominada método de predição via modelos de regressão, além de envolver baixo custo computacional, não anula a parte aleatória do modelo misto como no método MZ, pois estima os valores dos efeitos aleatórios individualmente para cada novo grupo.

Pela suposição do modelo (3.1), $\boldsymbol{\alpha}_{i}$ segue a distribuição normal multivariada. Então, cada marginal de efeitos aleatórios $\alpha_{m i}$, com $m=1, \ldots, k$ e $i=1, \ldots, q$, segue a distribuição normal univariada. Desta forma, um modelo linear, por exemplo, poderia ser utilizado para modelar individualmente cada marginal de efeitos aleatórios. Porém, empiricamente pode ocorrer destes efeitos não seguirem uma distribuição normal, e neste caso poderia ser utilizado um modelo nãoparamétrico para ajustar os dados não normais.

O procedimento do método é apresentado a seguir.

Na base de ajuste (BA) são obtidas as estimativas dos parâmetros do modelo logístico, e a função para predição da probabilidade da resposta $p_{i j}$ é dada por

$$
\hat{p}_{i j}=\frac{\exp \left\{\mathbf{x}_{i j}^{t} \hat{\boldsymbol{\beta}}+\mathbf{z}_{i j}^{t} \hat{\boldsymbol{\alpha}}_{i}\right\}}{1+\exp \left\{\mathbf{x}_{i j}^{t} \hat{\boldsymbol{\beta}}+\mathbf{z}_{i j}^{t} \hat{\boldsymbol{\alpha}}_{i}\right\}} .
$$

Como se tem o interesse em ajustar um modelo de regressão no nível de grupo, as covariáveis disponibilizadas no nível observação devem ser agregadas no nível de interesse por $\mathbf{w}_{i}^{t}=\left(\mathbf{x}_{i}^{t}, \mathbf{z}_{i}^{t}\right)$. Nota-se que $\mathbf{w}_{i}^{t}$ é o conjunto de todas as variáveis preditoras disponíveis no banco de dados agregadas no nível de grupo, que são funções das covariáveis associadas aos parâmetros fixos e aleatórios do modelo logístico misto. São exemplo de funções de agregação para variáveis contínuas: média, mediana, quartis, mínimo, máximo, entre outras; e para variáveis qualitativas: moda, primeira categoria, última categoria, entre outras.

Com todas as covariáveis agregadas no nível de grupo, busca-se uma função $f(\cdot)$ capaz de 
explicar os efeitos aleatórios estimados pelo modelo (3.50), dada por

$$
\hat{\alpha}_{m i}=f\left(\mathbf{w}_{m i}^{t} \boldsymbol{\lambda}_{m}\right),
$$

em que $i=1, \ldots, q$ indexa os grupos e $m=1, \ldots, k$ indexa o efeito aleatório. O vetor de coeficientes de regressão desconhecidos é $\boldsymbol{\lambda}_{m}=\left(\lambda_{m 1}, \lambda_{m 2}, \ldots, \lambda_{m p}\right)^{t}$, no qual $\lambda_{m 1}$ é o intercepto e $\left(\lambda_{m 2}, \ldots\right.$, $\left.\lambda_{m p}\right)$ são as inclinações, e $\mathbf{w}_{i}$ é o vetor de covariáveis conhecidas $(p \times q)$. A função $f(\cdot)$ pode ser definida por um modelo de regressão que seja capaz de explicar a variável resposta através das covariáveis. Observa-se que as estimativas dos parâmetros do modelo (3.51) foram obtidas na BA.

A predição para novos grupos é realizada na base de predição (BP), baseado nas estimativas do modelo (3.51), através da seguinte equação

$$
\hat{\alpha}_{m i}^{*}=f\left(\mathbf{w}_{m i}^{t} \hat{\boldsymbol{\lambda}}_{m}\right)
$$

Assim, por (3.52), na BP é possível obter o valor do efeito aleatório de um novo grupo, e por fim, predizer a probabilidade do evento de interesse de uma unidade pertencente a um $i$-ésimo novo grupo através de

$$
\hat{p}_{i j}=P\left(y_{i j}=1 \mid \boldsymbol{\alpha}_{i}^{*}\right)=\frac{\exp \left\{\mathbf{x}_{i j}^{t} \hat{\boldsymbol{\beta}}+\mathbf{z}_{i j}^{t} \hat{\boldsymbol{\alpha}}_{i}^{*}\right\}}{1+\exp \left\{\mathbf{x}_{i j}^{t} \hat{\boldsymbol{\beta}}+\mathbf{z}_{i j}^{t} \hat{\boldsymbol{\alpha}}_{i}^{*}\right\}},
$$

em que $\hat{\boldsymbol{\alpha}}_{i}^{*}=\left(\hat{\alpha}_{1 i}^{*}, \ldots, \hat{\alpha}_{k i}^{*}\right)$. Para a predição de novos efeitos aleatórios nas equações (3.52) e (3.53), utilizou-se a notação "asterisco"na função de interesse $\hat{\alpha}_{m i}^{*}$ para indicar o $i$-ésimo novo grupo, pertencentes à BP.

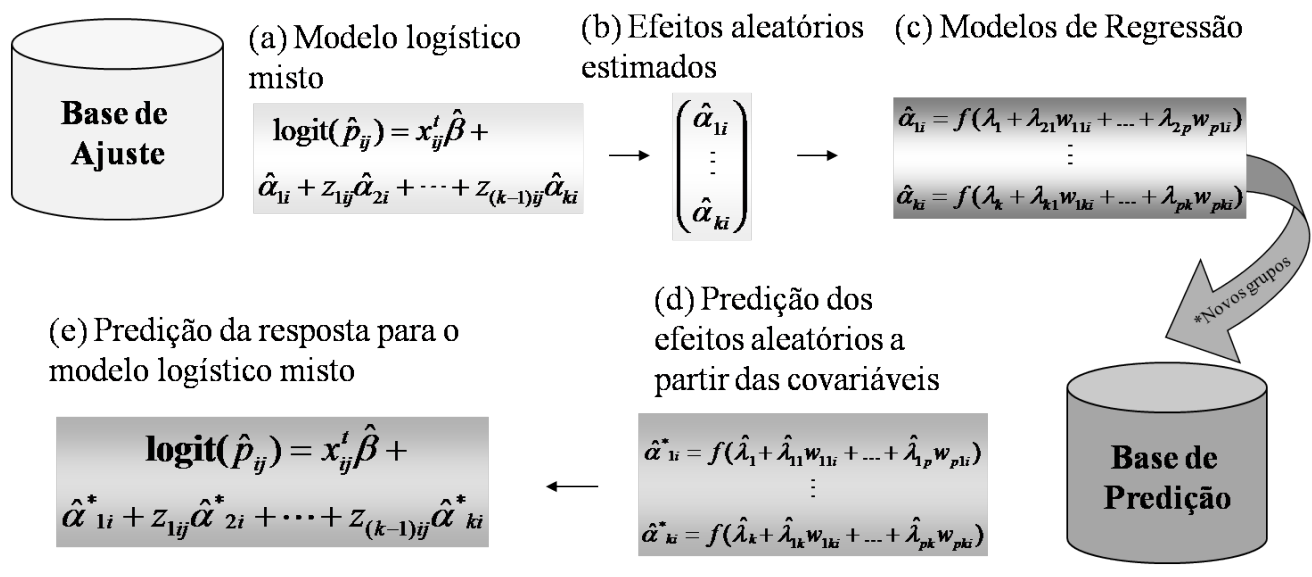

Figura 3.1: Resumo do método de predição via modelos de regressão.

Figura 3.1 resume os passos da metodologia de predição via modelos de regressão:

- Passo (a) Na base ajuste, obtenha as estimativas do modelo logístico misto, por (3.50).

- Passo (b) A partir da matriz de efeitos aleatórios estimados $(q \times k)$ pelo modelo (3.50), separe cada marginal de efeitos aleatórios $(q \times 1)$. Neste passo, agregue todas as covariáveis 
do banco de dados no nível de grupo, e obtenha $\mathbf{w}_{i}$.

- Passo (c) Para o $m$-ésimo efeito aleatório, ajuste um modelo de regressão, utilizando o vetor de covariáveis conhecidas $\mathbf{w}_{i}(p \times q)$. Segundo o método stepwise, selecione um modelo final para cada efeito aleatório.

- Passo (d) Na base de predição que considera novos grupos, calcule o valor dos novos efeitos $\left(\alpha_{m}^{*}\right)$, baseados nas covariáveis e nos parâmetros estimados em (c), segundo (3.52).

- Passo (e) Insira o valor do efeito aleatório predito obtido em (d) na função do modelo logístico misto, considerando os valores dos efeitos fixos estimados no passo (a) por (3.50). Finalmente, obtenha a probabilidade da resposta predita de uma observação pertencente a um novo grupo para o modelo logístico misto, segundo (3.53).

Nota-se que a metodologia proposta, usando o modelo (3.51), implica em $\alpha_{m i} \sim \mathcal{D}\left(\mathbf{w}_{m i}^{t} \boldsymbol{\lambda}_{\boldsymbol{m}}, \sigma_{m}^{2}\right)$, ou seja, ao invés de se assumir que a média de $\alpha_{i}$ é zero, nesta metodologia a média de $\alpha_{i m}=\mathbf{w}_{m i}^{t} \boldsymbol{\lambda}$, em que $\alpha_{i m}$ segue distribuição $\mathcal{D}$. Desta forma, supõe-se que os efeitos aleatórios podem ser explicados pelas características do grupo e não apenas pela variabilidade do grupo, como no caso do método MPE. Esta abordagem não leva em conta possíveis correlações entre os efeitos aleatórios.

Como cada marginal de efeitos aleatórios empíricos $\hat{\alpha}_{m}, \operatorname{com} m=1, \ldots, k$, pode ou não seguir uma distribuição normal, na literatura existem modelos distintos para ser utilizado em cada caso. A seguir são descritos o uso dos modelos de regressão adequados para cada situação.

\subsubsection{Método de predição via modelo linear}

Existem algumas situações práticas no qual o evento resposta, no caso o efeito aleatório estimado, pode ser modelado por uma função linear usando um conjunto de variáveis preditoras. Para abordar este tipo de estrutura de dados, o modelo linear pode ser utilizado. Este modelo tem sido amplamente utilizado em diversas aplicações, uma vez que o método de estimação é simples, além de estar implementado em praticamente todos os pacotes estatísticos.

Dada a suposição do modelo (3.2), parece ser razoável assumir que o modelo geral (3.51) possa ser particularizado para um modelo de regressão linear, dado por

$$
\hat{\alpha}_{m i}=\mathbf{w}_{m i}^{t} \boldsymbol{\lambda}_{m}+\varepsilon_{m i},
$$

com $\varepsilon_{m i} \sim \mathcal{N}\left(0, \sigma_{m}^{2}\right)$, independentes. No modelo (3.54), o parâmetro $\lambda_{m 1}$ é o intercepto e $\left(\lambda_{m 2}, \ldots, \lambda_{m p}\right)^{t}$ são as inclinações do vetor $\boldsymbol{\lambda}_{m}$. As estimativas dos parâmetros deste modelo podem ser obtidas pelo método de mínimos quadrados ou máxima verossimilhança, que são idênticas. Mais detalhes sobre o modelo de regressão linear podem ser vistos, por exemplo, em Draper et al. (1998). Nota-se que a variável resposta utilizada para estimação dos parâmetros de interesse do modelo (3.51) é $\hat{\alpha}_{m i}$, obtida pelo ajuste do modelo logístico (3.50). 
A fim de identificar um conjunto de covariáveis capaz de explicar a variável resposta (no caso, o efeito aleatório), vários métodos têm sido descritos na literatura para a seleção de modelos de regressão. Alguns métodos conhecidos como backward, forward, stepwise, $R^{2}$-ajustado, quadrado médio do resíduo $s^{2}$, Mallow $C_{P}$, Akaike Information Criterion (AIC), e da Bayes Information Criterion (BIC), podem ser usados. Para mais detalhes sobre estes procedimentos, vide Hocking (1976), Neter et al. (1996), Draper et al. (1998) ou Faraway (2002).

Estes métodos são úteis quando há muitos preditores no banco de dados. Neste trabalho, para se fazer esta seleção, utilizou-se o critério AIC, que é definido por

$$
A I C=-2 \log \text {-verossimilhança }+2 p,
$$

com $p$ representando o número de preditores no modelo em questão. Com o objetivo de minimizar o valor de AIC em cada passo de remoção dos preditores, a regra de parada está associada ao critério do p-valor, e pára quando todos os valores de $p$ são menores do que um valor crítico.

Assim, usando critério AIC no modelo (3.54), chega-se ao modelo final, em que agora $\hat{\lambda}_{m}$ $=\left(\hat{\lambda}_{m 1}, \ldots, \hat{\lambda}_{m p^{\prime}}\right)^{t}$ é o vetor de coeficientes de regressão estimados e $\mathbf{w}_{m i}^{t}$ é o vetor de covariáveis conhecido $\left(p^{\prime} \times 1\right)$, com $p^{\prime} \leq p$ sendo a quantidade de preditores presentes no modelo final.

Caso a técnica para a predição dos efeitos aleatórios seja a regressão linear, o passo (c) da Figura 3.1 é realizado utilizando esta técnica. Após a escolha do modelo final para cada efeito aleatório, finaliza-se a predição da probabilidade da variável resposta pelos passos (d) e (e).

\subsubsection{Método de Predição via modelos não-paramétricos}

Os modelos não-paramétricos podem ser usados como alternativa ao modelo linear paramétrico para o caso quando os efeitos aleatórios estimados não satisfizerem a suposição de normalidade. Desta forma, pode-se fazer a predição dos efeitos aleatórios de novos grupos utilizando-se um modelo não-paramétrico ou um modelo semi-paramétrico.

Modelos de regressão paramétricos, como por exemplo, o modelo de regressão linear (normal), que especifica a forma funcional do modelo antes de modelar os dados, sendo o objetivo estimar seus parâmetros. No caso dos modelos não-paramétricos, o objetivo é estimar uma função de regressão sem especificar sua forma funcional.

Nesta subseção são apresentados alguns tipos de modelos não-paramétricos. Uma descrição completa dos modelos não-paramétricos apresentados nesta seção são apresentados e discutidos em Hastie e Tibshirani (1990), Fox (2000a), Fox (2000b) e Weisberg e Fox (2010). Todos estes modelos ainda podem ser estendidos diretamente para regressão não-paramétrica generalizada, como apresentado no caso do modelo linear normal a extensão para os MLG, discutidos no Capítulo 2. Porém, como os efeitos aleatórios têm distribuição contínua, considerou-se o caso mais simples, sem generalizar esta classe de modelos.

Assim como na subseção anterior, nesta subseção considera-se o ajuste do modelo com a variável resposta $\hat{\alpha}_{m i}$, com $i=1, \ldots, q$ e $m=1, \ldots, k$, como descrito no passo (c) da Figura 3.1. Apenas 
por uma questão de simplificar a nomenclatura, os modelos serão apresentados com a variável resposta sem o acento circunflexo ("chapéu") e sem o índice $m$ para não se confundir com outras notações apresentadas mais adiante. Porém, considera-se que o valor da variável resposta, agora denominada por $\alpha_{i}$, é aquela estimada pelo modelo misto (3.50) do $i$-ésimo grupo para o $m$-ésimo efeito aleatório.

Um modelo de regressão não linear (paramétrico) ajusta um modelo do tipo

$$
\alpha_{i}=\lambda_{0}+\Upsilon\left(\mathbf{w}_{i}^{t}, \boldsymbol{\lambda}\right)+\varepsilon_{i}
$$

em que $\lambda_{0}$ é o intercepto, $\boldsymbol{\lambda}=\left(\lambda_{1}, \ldots, \lambda_{p}\right)$ é o vetor de parâmetros desconhecidos $(p \times 1)$ e $\mathbf{w}_{i}^{t}$ é o vetor de variáveis independentes conhecido $(1 \times p)$, com $\varepsilon_{i}$ erros i.i.d com média zero e variância constante $\sigma^{2}$ e $i=1, \ldots, q$. A função $\Upsilon(\cdot)$ que contêm os preditores é definida previamente, e se relaciona com média do valor da resposta $\alpha$ como no modelo de regressão linear tradicional (3.54). No modelo (3.55), $\Upsilon(\cdot)$ poderia ser funções do tipo: exponencial, logaritmo, raiz quadrada, entre outras, que são funções definidas previamente pelo usuário antes do ajuste do modelo.

O modelo de regressão não-paramétrico geral é escrito de uma maneira semelhante, como

$$
\alpha_{i}=\lambda_{0}+\Upsilon\left(w_{i 1}, w_{i 2}, \ldots, w_{i p}\right)+\varepsilon_{i}
$$

mas a função $\Upsilon(\cdot)$ é não especificada. O objetivo da regressão não-paramétrica é estimar a função de regressão $\Upsilon(\cdot)$ diretamente, em vez de estimar os parâmetros. A maioria dos métodos de regressão não-paramétricos implicitamente assumem que $\Upsilon(\cdot)$ é um função suave e contínua. Quando existem muitos preditores, modelos mais restritivos têm sido desenvolvidos. Um destes modelos é o modelo aditivo não-paramétrico, expressado por

$$
\alpha_{i}=\lambda_{0}+\Upsilon_{1}\left(w_{i 1}\right)+\Upsilon_{2}\left(w_{i 2}\right)+\ldots+\Upsilon_{p}\left(w_{i p}\right)+\varepsilon_{i},
$$

em que $\Upsilon_{c}(\cdot)$, com $c=1, \ldots, p$, são funções de suavização estimadas a partir dos dados. Este modelo é de fato mais restritivo em relação ao modelo não-paramétrico geral (3.56), mas menos restritivo do que o modelo de regressão não linear (3.55), que assume que todas as funções de regressão parciais são lineares em relação à resposta.

Variações do modelo aditivo incluem modelos semi-paramétricos, em que alguns dos preditores podem entrar no modelo de forma linear, como por exemplo,

$$
\alpha_{i}=\lambda_{0}+\lambda_{1} w_{i 1}+\Upsilon_{2}\left(w_{i 2}\right)+\ldots+\Upsilon_{p}\left(w_{i p}\right)+\varepsilon_{i}
$$

Em particular, neste trabalho optou-se por trabalhar com o modelo aditivo não-paramétrico e modelo aditivo semi-paramétrico com resposta contínua, respectivamente os modelos (3.57) e (3.58), que consideram erros normais e função de ligação identidade, e é indicado quando a regressão considera muitos preditores, conforme Hastie e Tibshirani (1990).

Existem diferentes maneiras para fazer estimação de modelos aditivos. O método mais geral 
para estimar os modelos aditivos considera o uso do algoritmo backfitting que permite estimar cada função por um suavizador arbitrário, por meio de um processo iterativo de ajuste.

A seguir, será revisado o processo de estimação da função de suavização do modelo aditivo que considera uma função de suavização univariada $\Upsilon(\cdot)$ para cada preditor, utilizando o algoritmo de backfitting.

Para resumir o algoritmo, será considerado o modelo aditivo (3.57), apesar do funcionamento do algoritmo ser equivalente para o modelo (3.58).

A média amostral fornece uma motivação intuitiva do algoritmo backfitting. Considere o modelo (3.57), e seja $\lambda^{(\iota)}=\bar{\alpha}^{(\iota)}$ a média amostral da variável modificada no passo $\iota$, com $\Upsilon_{1}^{0}, \ldots, \Upsilon_{p}^{0}$ sendo os valores iniciais de com $\Upsilon_{1}, \ldots, \Upsilon_{p}$ no início do algoritmo de backfitting. Cada iteração do algoritmo atualiza o valor de $\Upsilon_{c}^{0}$ para $\Upsilon_{c}^{1}$ por $\Upsilon_{c}^{0}=\Upsilon_{c}^{(\iota-1)}$, com $c=1, \ldots, p$.

Os valores de $\Upsilon_{c}^{1}$ são obtidos por meio de uma função de suavização $\mathbf{S}$. Exemplos de funções de suavização lineares incluem running mean, locally-weighted running-line, smoothing splines, loess smooth terms e kernel smoothers. Detalhes destas funções de suavização podem ser vistos no Capítulo 2 do livro de Hastie e Tibshirani (1990). Assim, calculam-se os resíduos parciais por

$$
\varepsilon_{i c}=\alpha_{i}^{(\iota)}-\bar{\alpha}_{i}^{(\iota)}-\sum_{c=1 ; c \neq c^{\prime}}^{p} \Upsilon_{c^{\prime}}^{0}\left(w_{i c^{\prime}}\right)
$$

com $i=1, \ldots, q$ e $c=1, \ldots, p$. O processo é repetido sequencialmente por

$$
\begin{gathered}
\Upsilon_{1}^{1}=\mathbf{S}_{1}^{(\iota)} \varepsilon_{1}^{(\iota)}=\mathbf{S}_{1}^{(\iota)}\left(\alpha^{(\iota)}-\mathbf{1} \bar{\alpha}^{(\iota)}-\Upsilon_{2}^{0}-\Upsilon_{2}^{0} \ldots-\Upsilon_{p}^{0}\right) \\
\Upsilon_{2}^{1}=\mathbf{S}_{2}^{(\iota)} \varepsilon_{2}^{(\iota)}=\mathbf{S}_{2}^{(\iota)}\left(\alpha^{(\iota)}-\mathbf{1} \bar{\alpha}^{(\iota)}-\Upsilon_{1}^{0}-\Upsilon_{3}^{0} \ldots-\Upsilon_{p}^{0}\right) \\
\vdots \\
\Upsilon_{p}^{1}=\mathbf{S}_{p}^{(\iota)} \varepsilon_{p}^{(\iota)}=\mathbf{S}_{p}^{(\iota)}\left(\alpha^{(\iota)}-\mathbf{1} \bar{\alpha}^{(\iota)}-\Upsilon_{1}^{0}-\Upsilon_{1}^{0} \ldots-\Upsilon_{p-1}^{0}\right),
\end{gathered}
$$

em que $\mathbf{S}_{c}^{(\iota)}$ tem dimensão $(q \times q)$ com $c=1, \ldots, p, \mathbf{1}$ é um vetor $(q \times 1)$ de valores unitários, e $\varepsilon_{i c}$ é o valor dos resíduos parciais, dado por (3.59).

O processo sequencial (3.60) pára quando todas as funções $\Upsilon_{c}^{1}$ convergirem por um critério pré-estabelecido.

A conexão entre o algoritmo de backfitting e o método de Gauss-Siedel torna-se mais clara quando se resolve o seguinte sistema de equações lineares:

$$
\left(\begin{array}{ccccc}
\mathbf{I} & \mathbf{S}_{1}^{(\iota)} & \mathbf{S}_{1}^{(\iota)} & \ldots & \mathbf{S}_{1}^{(\iota)} \\
\mathbf{S}_{2}^{(\iota)} & \mathbf{I} & \mathbf{S}_{2}^{(\iota)} & \ldots & \mathbf{S}_{2}^{(\iota)} \\
\vdots & \vdots & \vdots & \ddots & \vdots \\
\mathbf{S}_{p}^{(\iota)} & \mathbf{S}_{p}^{(\iota)} & \mathbf{S}_{p}^{(\iota)} & \ldots & \mathbf{I}
\end{array}\right) \quad\left(\begin{array}{c}
\Upsilon_{1}^{(\iota)} \\
\Upsilon_{2}^{(\iota)} \\
\vdots \\
\Upsilon_{p}^{(\iota)}
\end{array}\right)=\left(\begin{array}{c}
\mathbf{S}_{1}^{(\iota)} \alpha^{(\iota)} \\
\mathbf{S}_{2}^{(\iota)} \alpha^{(\iota)} \\
\vdots \\
\mathbf{S}_{p}^{(\iota)} \alpha^{(\iota)}
\end{array}\right)
$$


Em (3.61), o sistema possui $q p$ equações que correspondem aos $q p$ parâmetros a serem estimados.

A estatística AIC foi usada para fazer a seleção modelos, assim como no método RL, porém para os modelos não-paramétricos a estatística AIC foi baseada na distribuição Qui-quadrado, com graus de liberdade aproximados. O termo grau de liberdade é usualmente visto com uma escala contínua para a combinação de seleção de variáveis e a seleção dos parâmetros de suavização. O processo foi repetido até o número máximo de passos serem utilizados ou até o critério AIC não diminuir mais.

Caso a técnica para a predição dos efeitos aleatórios seja um modelo não-paramétrico, o passo (c) da Figura 3.1 é realizado utilizando esta técnica. Após a escolha do modelo final para cada efeito aleatório, finaliza-se a predição da variável resposta pelos passos (d) e (e).

Neste trabalho, foram utilizadas duas funções de suavização: smoothing splines e loess smooth terms. A seguir há uma breve descrição dos dois tipos de suavizadores e a suas respectivas matrizes suavizadoras $S_{c}^{(\iota)}$.

\section{Loess smooth terms}

O método de suavização loess (locally-weighted scatter plot), proposto por Cleveland (1979), baseia-se no ajuste de sucessivos modelos de regressão pelo método de mínimos quadrados ponderados de um conjunto de pontos $\left(\alpha_{i}, w_{i}\right)$, com $i=1, \ldots, q$, sendo $\alpha$ a variável resposta e $w$ representando uma variável explicativa.

Cada modelo é ajustado considerando observações cujo valor $w$ pertence a uma vizinhança da coordenada $w_{i}$ de uma observação $\left(\alpha_{i}, w_{i}\right)$, fixada que é denominada ponto alvo. Para cada ponto alvo $\left(\alpha_{i}, w_{i}\right)$ define-se uma vizinhança, e aos pontos $\left(\alpha_{i}, w_{i}\right)$ é ajustado um polinômio de grau $\varphi$, dado por $\alpha_{i^{\prime}}=\tau_{0}+\tau_{1} w_{i^{\prime}}+\ldots+\tau_{\varphi} w_{i^{\prime}}^{\varphi}+\varepsilon_{i^{\prime}}$, com $i^{\prime}=1, \ldots, q$, usando mínimos quadrados ponderados com pesos dados por uma função $\mathbf{V}$ a ser definida.

A função $\mathbf{V}$ que atribui os pesos em cada ajuste local do polinômio, tendo $\left(\alpha_{i}, w_{i}\right)$ como ponto alvo, é dada por

$$
\tilde{v}_{w_{i}, i^{\prime}}=\mathbf{V}\left(h_{i}^{-1}\left(w_{i^{\prime}}-w_{i}\right)\right)
$$

para $i^{\prime}=1, \ldots, q$, em que $h_{i}$ é a distância entre $w_{i}$ e seu $r$-ésimo vizinho mais próximo, i.e., coloca-se em ordem crescente as distâncias $w_{i}-w_{r^{\prime}}, \operatorname{com} r^{\prime}=1, \ldots, q$.

A função (3.62) depende da função tricúbica, dada por

$$
\tilde{\mathbf{V}}(\vartheta)=\left(1-\vartheta^{3}\right)^{3}
$$

para $0 \leq \vartheta \leq 1$ e zero, caso contrário. Com base na matriz de pesos (3.63), obtém-se a matriz de pesos referente ao ponto alvo $\left(\alpha_{i}, w_{i}\right)$, escrita como

$$
\mathbf{V}_{w_{i}}=\operatorname{diagonal}\left(v_{w_{i, 1}}, \ldots, v_{w_{i, q}}\right)
$$


com elementos dados por

$$
v_{w_{i}, i^{\prime}}=\left(1-\left|h_{i}^{-1}\left(w_{i^{\prime}}-w_{i}\right)\right|^{3}\right)^{3},
$$

para $\left|h_{i}^{-1}\left(w_{i^{\prime}}-w_{i}\right)\right|<1$, e zero, caso contrário.

Assim, por (3.64), em um ajuste local tendo como ponto alvo $\left(\alpha_{i}, w_{i}\right)$, o mesmo fica associado a um peso 1. Estes pesos diminuem à medida que os pontos se afastam de $\left(\alpha_{i}, w_{i}\right)$ e pontos fora da vizinhança de $w_{i}$ ficam associados a pesos nulos.

Posteriormente, realiza-se o ajuste do modelo regressão com o método de mínimos quadrados ponderados de $\alpha$ sobre $w$, usando a matriz (3.64). A primeira etapa termina após a realização deste procedimento para cada $w_{i}$. Assim, o valor ajustado $\hat{\alpha}_{i}$ em $w_{i}$ é o valor atribuído a $\hat{\Upsilon}\left(w_{i}\right)$, com $i=1, \ldots, q$.

No caso do suavizador loess, a $i$-ésima linha de $S_{c}^{(\iota)}$ corresponde à $i$-ésima linha da matriz suavizadora (3.60) por

$$
S_{c}^{(\iota)}=F\left(\mathbf{B}^{\prime} \mathbf{A B}\right)^{-1} \mathbf{B}^{\prime} \mathbf{A}
$$

em que $\mathbf{B}=\left(\mathbf{1}, \mathbf{B}_{c}\right), \mathbf{A}=\operatorname{diagonal}\left(v_{w_{1}, i^{\prime}} \vartheta_{w_{i, 1}}^{(m)}, \ldots, v_{w_{q}, i^{\prime}} \vartheta_{w_{i, q}}^{(m)}\right)$, com $v_{w_{i}, i^{\prime}}$ definido por (3.65) e $\vartheta_{c}^{(\iota)}=\operatorname{diagonal}\left(\vartheta_{w_{i, 1}}, \ldots, \vartheta_{w_{i, q}}\right)$.

\section{Smothing splines}

Um suavizador spline é uma função $\hat{\Upsilon}$ que minimiza:

$$
S S(\Upsilon)=\sum_{i=1}^{n}\left[\alpha_{i}-\Upsilon\left(w_{i}\right)\right]^{2}+\psi \int_{w_{\max }}^{w_{\min }}\left[\Upsilon^{\prime \prime}(w)\right]^{2} d w
$$

sobre todas as funções $\Upsilon$ com primeiras e segundas derivadas contínuas em um intervalo [ $\left.w_{\min }, w_{\max }\right]$. Reinsch (1967) e Green e Silverman (1993) provaram a existência e unicidade de $\hat{\Upsilon}$. O parâmetro $\psi$ é responsável por regular a suavização da curva: valores altos de $\psi$ dão um peso maior à segunda parcela da equação (3.67), produzindo curvas mais suaves. Quando $\psi \rightarrow \infty$ tem-se $\left[\Upsilon^{\prime \prime}(w)\right]^{2}=0$, e então a solução é a reta de mínimos quadrados. No outro extremo, quando $\psi \rightarrow 0$, a segunda parcela de (3.67) tem pouca influência, e a solução tende a ser uma função duas vezes diferenciável que interpola os $q$ pontos.

A expressão (3.67) é denominada cubic splines, em que o primeiro termo da expressão mede a bondade do ajuste pela soma de quadrados dos resíduos e o segundo termo penaliza a curvatura da função. Nesta mesma expressão, $\int_{w_{\max }}^{w_{\min }}\left[\Upsilon^{\prime \prime}(w)\right]^{2} d w$ mede a ondulação ou a suvização da função $\Upsilon$, em que para funções lineares $\int_{w_{\max }}^{w_{\min }}\left[\Upsilon^{\prime \prime}(w)\right]^{2} d w=0$ e para funções não lineares $\int_{w_{\max }}^{w_{\min }}\left[\Upsilon^{\prime \prime}(w)\right]^{2} d w>$ 0 .

Fahrmeir et al. (1994) provaram que minimizar $S S(h)$ é equivalente a minimizar

$$
(\alpha-\Upsilon)^{\prime}(\alpha-\Upsilon)+\psi \Upsilon^{\prime} \mathbf{K} \Upsilon
$$

em que $\alpha$ é o vetor de observações de variáveis resposta, $\mathbf{K}$ é uma matriz $(q \times q) \operatorname{com} \mathbf{K}=\mathbf{D}^{\prime} \mathbf{M}^{-1} \mathbf{D}$. 
A matriz tridiagonal superior $\mathbf{D}$ com dimensão $((q-2) \times n)$ é definida pelos elementos:

$$
\begin{gathered}
d_{i i}=l_{i}^{-1}, \\
d_{i(i+1)}=-\left(l_{i}^{-1}+l_{(i+1)}^{-1}\right)
\end{gathered}
$$

$\mathrm{e}$

$$
d_{i(i+2)}=l_{i+1}^{-1},
$$

para $i=1, \ldots, q-2$, e zero para as demais caselas da matriz. $\mathbf{M}$ é uma matriz simétrica tridiagonal $((q-2) \times(q-2))$ definida por

$$
m_{i i}=2\left(l_{i}+l_{i+1}\right)
$$

para $i=1, \ldots, q-2, \mathrm{e}$

$$
m_{(i-1) i}=m_{i(i-1)}=l_{i},
$$

para $i=1, \ldots, q-2$, e zero para as demais caselas da matriz.

Para minimizar (3.68) se faz derivada com respeito a $\Upsilon$, e igualando a zero, obtém-se a solução

$$
\hat{\Upsilon}=(\mathbf{I}+u \mathbf{K})^{-1} \alpha=S \alpha
$$

em que I é a matriz identidade $(q \times q)$ e $\mathbf{S}=(\mathbf{I}+u \mathbf{K})$ é a matriz suavizadora. Os elementos desta matriz dependem apenas de $w_{1}, \ldots, w_{q}$ e do parâmetro de suavização $\psi$, e portanto cubic splines também é um suavizador linear. Quanto menor o valor de $\psi$, menores serão os valores dos elementos da diagonal da matriz $S$ e menores os valores dos elementos fora desta diagonal. Os valores absolutos destes elementos diminuem à medida que eles se afastam da diagonal.

No caso do suavizador smothing splines, a $i$-ésima linha de $S_{c}^{(\iota)}$ corresponde à $i$-ésima linha da matriz suavizadora $(3.60)$

$$
S_{c}^{(\iota)}=\left(\mathbf{E}+\psi_{c} \mathbf{K}_{c}\right)^{-1} \mathbf{E},
$$

em que $\mathbf{E}=\operatorname{diagonal}\left(v_{w_{i, 1}}, \ldots, v_{w_{i, q}}\right)$ é a diagonal de pesos, $\mathbf{K}_{c}$ é a matriz de penalização para a $c$-ésima covariável, definida por $\mathbf{K}=\mathbf{D}^{\prime} \mathbf{M}^{-1} \mathbf{D}$, com $\mathbf{M}$ a matriz tridiagonal definida anteriormente, e $\psi_{c}$ é o parâmetro de suavização fixado. 


\section{Capítulo 4}

\section{Estudos de simulação}

O objetivo deste capítulo é comparar os diferentes métodos de estimação e predição, apresentados no Capítulo 3, por meio de estudos de simulação. Para avaliar a predição dos métodos de predição foram utilizados os resíduos da predição, adicionalmente às medidas AUC e KS apresentadas no Capítulo 2, que serão definidos neste capítulo.

Sem perda de generalidade, os dados simulados foram gerados a partir do modelo de logístico misto, dado por

$$
p_{i j}=\operatorname{logit}\left[P\left(y_{i j}=1 \mid\left(\alpha_{1 i}, \alpha_{2 i}\right)^{t}\right)\right]=\beta x_{i j}+\alpha_{1 i}+\alpha_{2 i} x_{i j},
$$

em $y_{i j}$ é resposta binária da $j$-ésima observação dentro $i$-ésimo grupo, com $i=1,2, \ldots, q$ e $j=$ $1,2, \ldots, n, x_{i j}$ é uma covariável conhecida e $\beta$ é um parâmetro fixo desconhecido. Assume-se que os efeitos aleatórios $\left(\alpha_{1 i}, \alpha_{2 i}\right)^{t}$ são parâmetros desconhecidos, que seguem uma distribuição normal bivariada, com vetor de médias igual a zero e matriz de variância-covariância igual a $\boldsymbol{\Sigma}$. Os elementos desconhecidos de $\boldsymbol{\Sigma}$ são: $\sigma_{1}^{2}$ (variância do intercepto aleatório), $\sigma_{12}=\sigma_{21}$ (covariância entre os efeitos aleatórios) e $\sigma_{2}^{2}$ (variância da inclinação aleatória).

Os estudos de simulação foram divididos em quatro partes. Em cada estudo considerou-se a comparação entre diferentes cenários, em que foram assumidos valores distintos no modelo (4.1) para: o tamanho do grupo, o desvio-padrão dos efeitos aleatórios, a correlação entre os efeitos aleatórios, e o valor do efeito fixo; totalizando em 7 cenários, conforme Tabela 4.1.

Nesta tabela, $n$ representa o tamanho do grupo; $N$, a quantidade de observações do conjunto de dados, com $N=n q ; \beta$, o parâmetro fixo; $\sigma_{1}$, o desvio-padrão do intercepto aleatório; $\sigma_{2}$, o desvio-padrão da inclinação aleatória; $\sigma_{12}$, a covariância entre os efeitos aleatórios; e $\rho$, a correlação entre os efeitos aleatórios, $\operatorname{com} \rho=\sigma_{12} / \sigma_{1} \sigma_{2}$.

Tabela 4.1: Cenários de simulação.

\begin{tabular}{crrrrrrr}
\hline Cenário & $n$ & $N$ & $\beta$ & $\sigma_{1}$ & $\sigma_{2}$ & $\sigma_{12}$ & $\rho$ \\
\hline 1 & 3 & 120 & 3 & 5,0 & 5,0 & 12,500 & 0,50 \\
2 & 6 & 120 & 3 & 5,0 & 5,0 & 12,500 & 0,50 \\
3 & 12 & 120 & 3 & 5,0 & 5,0 & 12,500 & 0,50 \\
4 & 6 & 120 & 3 & 0,5 & 0,5 & 1,125 & 0,50 \\
5 & 6 & 120 & 3 & 5,0 & 5,0 & 3,750 & 0,15 \\
6 & 6 & 120 & 3 & 5,0 & 5,0 & 21,500 & 0,85 \\
7 & 6 & 120 & 1 & 5,0 & 5,0 & 12,500 & 0,50 \\
\hline
\end{tabular}


O primeiro estudo de simulação compara os cenários 1, 2 e 3, com os tamanhos de grupos $(n)$ assumindo os valores 3, 6 e 12, respectivamente; e tem o objetivo de verificar se a variação do tamanho dos grupos influencia no viés da estimativa dos parâmetros e na predição da resposta, para os valores constantes de: $N, \beta, \sigma_{1}, \sigma_{2}, \sigma_{12}$ e $\rho$.

O segundo estudo de simulação considera a comparação entre os cenários 4 e 2 , em que os valores do desvio-padrão do intercepto e do desvio-padrão da inclinação aleatória $\left[\sigma_{1} ; \sigma_{2}\right]$ assumem os valores $[0,5 ; 0,5]$ e $[5,0 ; 5,0]$; e tem o objetivo de verificar se a variação nos valores $\left[\sigma_{1} ; \sigma_{2}\right]$ influencia no viés da estimativa dos parâmetros e na predição da resposta, para os valores constantes de: $n$, $N, \beta, \sigma_{12}$ e $\rho$.

A comparação realizada para o terceiro estudo de simulação tem o objetivo de avaliar mudança de patamar da covariância entre os efeitos aleatórios $\left(\sigma_{12}\right)$, que é função de $\rho$; e verificar se a variação dos valores de $\sigma_{12}$ influencia no viés da estimativa dos parâmetros e na predição da resposta, para os valores constantes de: $n, N, \beta, \sigma_{1}$ e $\sigma_{2}$. Para este fim, foram analisados os cenários 5,2 e 6 , com $\left[\sigma_{12} ; \rho\right]$ assumindo os valores $[3,750 ; 0,15],[12,500 ; 0,50]$ e $[21,125 ; 0,85]$, respectivamente.

O quarto e último estudo de simulação compara os cenários 7 e 2 , com $\beta$ assumindo os valores 1 e 3; e tem o objetivo de verificar se a variação dos valores do parâmetro da parte fixa $(\beta)$ do modelo influencia no viés da estimativa dos parâmetros e na predição da resposta, para os valores constantes de: $n, N, \sigma_{1}, \sigma_{2}, \sigma_{12}$ e $\rho$.

Para cada cenário simulado, foram avaliadas as estimativas dos efeitos fixos e dos desvios-padrão dos efeitos aleatórios, pelos métodos de estimação QVP, AL e QAGH. Essas estimativas foram utilizadas para a predição da probabilidade da resposta pelos métodos de predição MZ, MPE, RL e NP, com o objetivo de identificar em quais cenários de simulação os métodos de predição propostos são mais adequados.

Uma vez que nos estudos de simulação conhece-se a probabilidade verdadeira calculada a partir de (4.1), é possível criar índices de desempenho em termos de predição da resposta, baseados em resíduos. Assim, foram calculados os resíduos: resíduo ao quadrado, dado por $r e s_{i j}^{2}=\left(\hat{p}_{i j}-p_{i j}\right)^{2}$; e resíduos absolutos, dado por $\operatorname{res}_{i j}=\left|\hat{p}_{i j}-p_{i j}\right|$, com $\hat{p}_{i j}$ sendo a probabilidade predita da $j$-ésima observação dentro do $i$-ésimo novo grupo; e $p_{i j}$, a probabilidade verdadeira da $j$-ésima observação dentro do $i$-ésimo novo grupo. O intervalo de valores que $r e s_{i j}^{2}$ e $r e s_{i j}$ podem assumir está entre zero e um, sendo que valores mais próximos de zero indicam boa predição, e consequentemente valores mais próximos de um indicam baixa predição.

As medidas utilizadas para avaliar a predição da resposta para novos grupos foram os índices AUC e KS, descritos no Capítulo 2, e os resíduos da predição, descritos no parágrafo anterior. Observa-se que os índices AUC e KS avaliam a predição em termos da classificação da resposta binária (comparação entre as observações), enquanto os resíduos da predição avaliam a distância entre o valor da probabilidade predita em relação a seu valor verdadeiro (comparação dentro da observação).

Para complementar a análise dos métodos de predição, os estudos de simulação consideraram 
também o ajuste do modelo logístico tradicional, que ignora a presença dos efeitos aleatórios, dado por

$$
\operatorname{logit}\left[P\left(y_{i j}=1\right)\right]=\beta x_{i j} .
$$

O ajuste do modelo (4.2) foi realizado na mesma base de dados gerada pelo modelo (4.1). O objetivo desta comparação é verificar a qualidade da predição para novos grupos a partir do modelo tradicional e dos métodos de predição baseados no modelo misto.

Na próxima seção são descritos os passos do esquema de simulação.

\subsection{Esquema de simulação}

Sem perda de generalidade, os valores da covariável $x$ foram gerados a partir da distribuição normal com média zero e variância um. A geração dos valores de $x$ foi realizada por grupo, o qual era composto por $n$ observações, e a quantidade de grupos era igual a $q$, totalizado $N$ valores de $x$.

Com $x$ fixado inicialmente, foram gerados 1.000 conjuntos de dados (réplicas) de tamanho $N$.

Para cada réplica, foram gerados os valores dos efeitos aleatórios por grupo, seguindo uma distribuição normal bivariada, com vetor de médias igual a zero e matriz de covariância igual a $\boldsymbol{\Sigma}$. A probabilidade do evento resposta ou sucesso para cada unidade amostral foi calculada pelo modelo (4.1), e com base nesta probabilidade, gerou-se a resposta binária a partir da distribuição Bernoulli.

A fim de avaliar a predição para novos grupos, cada conjunto de dados foi separado em duas partes: base de ajuste (BA) e base de predição (BP). Para tal, a amostragem foi feita por grupo, da seguinte forma: os grupos indexados por números pares foram selecionados para a BA, enquanto os grupos indexados pelos números ímpares foram selecionados para a BP. Portanto, cada base foi composta por $N / 2$ observações com $q / 2$ grupos.

As estimativas dos parâmetros do modelo foram obtidas a partir da BA, pelo modelo tradicional e pelos métodos de estimação do modelo misto QVP, AL e QAGH para 2 e 5 pontos na quadratura. Baseadas nessas estimativas, a predição da resposta para o modelo misto foi realizada pelos métodos de predição MZ, MPE, RL e NP. Especificamente, o método MPE aproximou yi. por (3.49), e o método NP considerou o suavizador loess, dado por (3.66). No caso do modelo tradicional, a predição foi realizada pela função logito.

Assim, para cada réplica, ajustou-se o modelo tradicional e misto, guardando-se as estimativas dos parâmetros fornecidas pelos diversos métodos de estimação, e as probabilidades preditas pelos respectivos métodos de estimação e predição.

Ao final de cada réplica, guardaram-se os valores de AUC e KS das BA e BP, e o valor da mediana dos resíduos da BP. Utilizou-se a mediana como medida de resumo de centralidade, pois os resíduos gerados pelo modelo misto apresentaram assimetria acentuada à direita, enquanto o modelo tradicional apresentou distribuição dos resíduos simétrica.

Este procedimento foi repetido até que se completassem as 1.000 réplicas desejadas.

Posteriormente, foi calculado o viés médio das estimativas e o percentual de variação da média 
da estimativa em relação ao seu valor verdadeiro, para cada parâmetro do correspondente método de estimação. Também, calculou-se o valor médio de AUC e KS, e a mediana das medianas dos resíduos ao quadrado e dos resíduos absolutos. Denominou-se Md.res.qua a mediana das medianas dos resíduos ao quadrado, e Md.res.abs a mediana das medianas dos resíduos absolutos.

Todos os métodos de estimação do modelo logístico misto apresentaram alguns problemas computacionais durante o processo de simulação. Desta forma, a quantidade de problemas como falta de convergência na estimação dos parâmetros e estimativas dos parâmetros não plausíveis (valor de desvio-padrão do efeito aleatório zero, nulo ou infinito) foram contabilizadas até que se completassem as 1.000 réplicas desejadas. Mais detalhes sobre estes problemas são apresentados na Seção 4.3 .

A seguir, é descrito resumidamente o esquema usado para simular os cenários dos estudos de simulação.

Início do algoritmo

Passo 0: entra-se com os valores iniciais de $n, N, \beta, \sigma_{1}, \sigma_{2}$ e $\sigma_{12}$

Passo 1: gera-se o valor da covariável $x$

Início das réplicas

Passo 2: gera-se o valor dos efeitos aleatórios a partir de uma Normal Bivariada

Passo 3: calcula-se a probabilidade verdadeira pelo modelo (4.1)

Passo 4: gera-se a variável resposta baseado no Passo 3

Passo 5: separa-se a base de dados em BA e BP

Passo 6: ajusta-se o modelo tradicional e misto pelos métodos de estimação QVP, AL, QAGH=2 e

$\mathrm{QAGH}=5$ na $\mathrm{BA}$

Passo 7: checa-se se todos os métodos de estimação foram ajustados sem apresentar problemas na estimação dos parâmetros

Passo 8: faz-se a predição da resposta na BP, pelos métodos tradicional, MZ, MPE, RL e NP

Passo 9: guarda-se o valor das estimativas dos parâmetros, e dos valores de AUC, KS, mediana de res $^{2}$ e a mediana de res

Fim das réplicas

Passo 10: Sob as 1.000 réplicas, calculam-se a média das estimativas dos parâmetros, a média de AUC e KS, e finalmente, Md.res.qua e Md.res.abs

Fim do algoritmo-

As próximas seções deste capítulo apresentam os resultados dos cenários simulados e os aspectos computacionais envolvidos nos estudos de simulação.

\subsection{Análise dos resultados}

Nesta seção são apresentados os resultados dos estudos de simulação.

Primeiramente, avaliou-se o viés das estimativas dos parâmetros do modelo tradicional e misto, pela média da estimativa e pelo percentual de variação da média da estimativa em relação a seu 
valor verdadeiro. Posteriormente, o desempenho preditivo na BA e BP foram avaliados pela média dos valores de AUC e KS. Adicionalmente para a BP, analisou-se a mediana dos resíduos da predição Md.res.qua e Md.res.abs.

\subsubsection{Estimativa dos parâmetros}

Nesta subseção são discutidos os resultados das estimativas dos parâmetros do modelo tradicional e misto, para cada método de estimação, para os diversos cenários de simulação. Os métodos de estimação utilizados para o modelo misto foram: QVP, AL e QAGH para 2 e 5 pontos na quadratura, descritos no Capítulo 3.

Segundo Pinheiro e Bates (1995) a aproximação de Laplace, ou equivalentemente QAGH com 1 ponto na quadratura, fornece uma aproximação razoável. No caso da quadratura adaptativa de Gauss-Hermite, Pinheiro e Chao (2006) discutem que 7 pontos ou menos na quadratura são suficientes na prática e menos complexos computacionalmente, uma vez que quanto maior a quantidade de pontos na quadratura melhor é a aproximação do método. Pinheiro e Chao (2006) simularam modelos com 3, 5 e 7 pontos na quadratura, e os resultados mostram que 5 pontos na quadratura fornece suficiente acurácia para aproximação do método. No presente trabalho, foi feito um pequeno estudo de simulação para 2 e 3 pontos na QAGH, e como os resultados foram similares, optou-se por trabalhar com 2 pontos, devido a menor complexidade computacional. Desta forma, a escolha de 2 e 5 pontos na quadratura nos estudos de simulação deste capítulo foi baseada no artigo de Pinheiro e Chao (2006).

No caso do modelo tradicional, foi usado o método de máxima verossimilhança, e denotou-se o método deste modelo como "Tradicional" na apresentação dos resultados.

A Tabela 4.2 apresenta a média das estimativas e o percentual de variação da média das estimativas em relação aos valores verdadeiros dos parâmetros $\beta, \sigma_{1}, \sigma_{2}$ e $\sigma_{12}$, para cada cenário de simulação. O percentual de variação é apresentado entre parênteses, ao lado da média da estimativa de cada parâmetro.

Analisando os resultados desta tabela, observa-se que a média das estimativas dos parâmetros $\beta, \sigma_{1}, \sigma_{2}$ e $\sigma_{12}$ foram, no geral, subestimadas em relação ao verdadeiro valor, para todos os métodos de estimação.

O método de estimação do modelo tradicional forneceu maior viés na estimativa do parâmetro $\beta$ comparado com os métodos de estimação do modelo misto. Comparando os vieses das estimativas de $\beta$ entre os métodos de estimação do modelo misto, no geral, os vieses de QVP e AL foram menores do que as fornecidas pela QAGH com 5 e 2 pontos na quadratura.

No caso dos parâmetros $\sigma_{1}$, o método $\mathrm{AL}$ foi aquele que apresentou estimativa média dos parâmetros com menor viés. Para o parâmetro $\sigma_{2}$, os métodos que apresentaram menor viés, no geral, foram QVP e AL. Por fim, o método AL foi aquele que apresentou menor viés na estimativa do parâmetro $\sigma_{12}$, e o QVP aquele que apresentou, no geral, maior viés.

A seguir, são analisados em detalhes os resultados dos vieses das estimativas dos parâmetros, 
Tabela 4.2: Média (percentual de variação em relação ao verdadeiro valor) das estimativas dos parâmetros $\beta, \sigma_{1}, \sigma_{2}$ e $\sigma_{12}$, por método de estimação, baseado no resultado de 1.000 réplicas.

\begin{tabular}{|c|c|c|c|c|c|}
\hline \multirow{2}{*}{ Cenário } & \multirow{2}{*}{ Método de Estimação } & \multicolumn{4}{|c|}{ Média (percentual de variação) das estimativas dos parâmetros } \\
\hline & & $\beta$ & $\sigma_{1}$ & $\sigma_{2}$ & $\sigma_{12}$ \\
\hline \multirow{5}{*}{1} & Tradicional & $0,55(-81,7 \%)$ & & & \\
\hline & QVP & $1,98(-34,1 \%)$ & $3,90(-22,0 \%)$ & $4,24(-15,2 \%)$ & $2,12(-83,0 \%)$ \\
\hline & $\mathrm{AL}$ & $3,06(1,9 \%)$ & $4,86(-2,9 \%)$ & $5,09(1,9 \%)$ & $9,60(-23,2 \%)$ \\
\hline & $\mathrm{QAGH}=2$ & $2,08(-30,6 \%)$ & $3,53(-29,3 \%)$ & $3,26(-34,8 \%)$ & $6,30(-49,6 \%)$ \\
\hline & $\mathrm{QAGH}=5$ & $1,90(-36,6 \%)$ & $3,23(-35,5 \%)$ & $2,87(-42,6 \%)$ & $4,83(-61,4 \%)$ \\
\hline \multirow{5}{*}{2} & Tradicional & $0,62(-79,2 \%)$ & & & \\
\hline & QVP & $2,65(-11,8 \%)$ & $4,09(-18,3 \%)$ & $4,93(-1,5 \%)$ & $5,91(-52,8 \%)$ \\
\hline & $\mathrm{AL}$ & $2,80(-6,5 \%)$ & $4,79(-4,1 \%)$ & $4,48(-10,4 \%)$ & $9,08(-27,4 \%)$ \\
\hline & $\mathrm{QAGH}=2$ & $2,42(-19,4 \%)$ & $4,23(-15,3 \%)$ & $3,69(-26,2 \%)$ & $7,06(-43,5 \%)$ \\
\hline & $\mathrm{QAGH}=5$ & $2,30(-23,3 \%)$ & $4,04(-19,2 \%)$ & $3,46(-30,8 \%)$ & $6,27(-49,8 \%)$ \\
\hline \multirow{5}{*}{3} & Tradicional & $0,60(-79,9 \%)$ & & & \\
\hline & QVP & $2,51(-16,4 \%)$ & $4,07(-18,7 \%)$ & $4,56(-8,7 \%)$ & $6,04(-51,7 \%)$ \\
\hline & $\mathrm{AL}$ & $2,31(-22,9 \%)$ & $4,60(-8,0 \%)$ & $4,27(-14,5 \%)$ & $8,35(-33,2 \%)$ \\
\hline & $\mathrm{QAGH}=2$ & $2,21(-26,3 \%)$ & $4,38(-12,4 \%)$ & $3,94(-21,3 \%)$ & $7,43(-40,6 \%)$ \\
\hline & $\mathrm{QAGH}=5$ & $2,14(-28,6 \%)$ & $4,24(-15,3 \%)$ & $3,77(-24,7 \%)$ & $6,82(-45,4 \%)$ \\
\hline \multirow{5}{*}{4} & Tradicional & $2,76(-8,0 \%)$ & & & \\
\hline & QVP & $3,17(5,7 \%)$ & $0,82(64,0 \%)$ & $0,69(38,6 \%)$ & $0,02(-99,8 \%)$ \\
\hline & $\mathrm{AL}$ & $3,10(3,4 \%$ & $0,51(1,6 \%)$ & $0,48(-3,4 \%)$ & $0,02(-99,8 \%)$ \\
\hline & $\mathrm{QAGH}=2$ & $3,09(3,0 \%$ & $1,8 \% \quad)$ & $0,46(-9,0 \%)$ & $0,02(-99,8 \%)$ \\
\hline & $\mathrm{QAGH}=5$ & $3,09(3,0 \%)$ & $1,8 \% \quad)$ & $0,46(-8,6 \%)$ & $0,02(-99,8 \%)$ \\
\hline \multirow{5}{*}{5} & Tradicional & $0,58(-80,6 \%)$ & & & \\
\hline & QVP & $2,18(-27,5 \%)$ & $3,51(-29,8 \%)$ & $4,11(-17,7 \%)$ & $0,85(-77,4 \%)$ \\
\hline & $\mathrm{AL}$ & $2,37(-20,9 \%)$ & $4,06(-18,7 \%)$ & $3,73(-25,4 \%)$ & $0,94(-74,8 \%)$ \\
\hline & $\mathrm{QAGH}=2$ & $2,12(-29,3 \%)$ & $3,69(-26,2 \%)$ & $3,13(-37,3 \%)$ & $0,80(-78,6 \%)$ \\
\hline & $\mathrm{QAGH}=5$ & $2,03(-32,3 \%)$ & $3,58(-28,5 \%)$ & $2,99(-40,2 \%)$ & $0,80(-78,7 \%)$ \\
\hline \multirow{5}{*}{6} & Tradicional & $0,76(-74,6 \%)$ & & & \\
\hline & QVP & $3,08(2,6 \%)$ & $4,24(-15,2 \%)$ & $4,81(-3,8 \%)$ & $14,36(-32,0 \%)$ \\
\hline & $\mathrm{AL}$ & $2,83(-5,7 \%)$ & $4,83(-3,5 \%)$ & $4,78(-4,4 \%)$ & $21,12(0,0 \%)$ \\
\hline & $\mathrm{QAGH}=2$ & $2,59(-13,6 \%)$ & $4,42(-11,5 \%)$ & $4,19(-16,1 \%)$ & $17,65(-16,4 \%)$ \\
\hline & $\mathrm{QAGH}=5$ & $2,50(-16,6 \%)$ & $4,22(-15,7 \%)$ & $3,94(-21,1 \%)$ & $15,51(-26,6 \%)$ \\
\hline \multirow{5}{*}{7} & Tradicional & $0,21(-78,6 \%)$ & & & \\
\hline & QVP & $0,93(-7,1 \%)$ & $4,00(-20,0 \%)$ & $4,84(-3,3 \%)$ & $6,62(-45,4 \%)$ \\
\hline & $\mathrm{AL}$ & $0,93(-7,2 \%)$ & $4,73(-5,5 \%)$ & $4,46(-10,8 \%)$ & $10,45(-13,8 \%)$ \\
\hline & $\mathrm{QAGH}=2$ & $0,83(-17,3 \%)$ & $4,21(-15,9 \%)$ & $3,79(-24,2 \%)$ & $8,09(-33,3 \%)$ \\
\hline & $\mathrm{QAGH}=5$ & $0,79(-20,8 \%)$ & $3,98(-20,3 \%)$ & $3,56(-28,9 \%)$ & $7,02(-42,1 \%)$ \\
\hline
\end{tabular}

por estudo de simulação.

\section{Estudo 1 - Resultados da variação do tamanho do grupo (cenários: 1, 2 e 3)}

Os resultados indicaram que quando o tamanho do grupo $n$ aumentou de 3 para 12, o viés da estimativa de $\beta$ diminui no caso de AL. Nos outros métodos de estimação não se observou um padrão. Por exemplo, para o método AL, o viés pareceu aumentar pela análise do percentual de variação. Para os demais métodos, o viés diminuiu de $n=3$ para $n=6$, mas aumentou de $n=6$ para $=12$. No caso dos parâmetros $\sigma_{1}, \sigma_{2}$ e $\sigma_{12}$ segundo a média de seus valores estimados e o 
percentual de variação o viés diminuiu quando $n$ aumentou para todos os métodos de estimação, com exceção de AL que apresentou um comportamento contrário.

\section{Estudo 2 -Resultados da variação de $\sigma_{1}$ e $\sigma_{2}$ (cenários: 4 e 2)}

No geral, os resultados indicaram que quando o valor do desvio-padrão dos efeitos aleatórios $\left[\sigma_{1} ; \sigma_{2}\right]$ aumentaram de $[0,5 ; 0,5]$ para $[5,0 ; 5,0]$, segundo a média das estimativas de $\beta, \sigma_{1}$ e $\sigma_{2}$, pela análise do percentual de variação, o viés aumentou para todos os métodos de estimação. A exceção ocorreu para método QVP, referente aos parâmetros estimados $\sigma_{1}$ e $\sigma_{2}$. Para o parâmetro $\sigma_{12}$, pela análise do percentual de variação o viés diminuiu, para todos os métodos de estimação.

Comparando os cenários 4 e 2, conforme o esperado, nota-se que o modelo misto é o mais adequado quando os valores dos desvios-padrão dos efeitos aleatórios são distantes de zero. Para valores próximos de zero, o viés da estimativa de $\beta$ para o modelo tradicional foi similar ao apresentado pelos métodos de estimação do modelo misto. Isso ocorreu no cenário 4, pois a parte aleatória do modelo misto apresentou uma menor importância no preditor linear devido à baixa variabilidade dos efeitos aleatórios.

\section{Estudo 3 -Resultados da variação de $\sigma_{12}$ (cenários: 5, 2 e 6 )}

Os resultados indicaram que quando o valor de $\sigma_{12}$ aumentou (ou $\rho$ aumentou de 0,15 para 0,85), a média dos valores estimados de $\beta, \sigma_{1}, \sigma_{2}$ e $\sigma_{12}$ tornaram-se mais próximas dos verdadeiros valores, assim pela análise do percentual de variação o viés diminuiu para todos os métodos de estimação.

\section{Estudo 4 - Resultados da variação de $\beta$ (cenários: 7 e 2)}

Os resultados indicaram que quando o valor de $\beta$ aumentou de 1 para 3 , o viés aumentou, segundo a análise do percentual de variação. Para os parâmetros $\sigma_{1}$ e $\sigma_{2}$, a média de suas estimativas apresentaram valores muito próximos quando comparados os cenários 7 e 2, portanto os vieses das estimativas dos parâmetros analisando o percentual de variação resultaram em valores no mesmo patamar. Por fim, a média dos valores estimados de $\sigma_{12}$ afastou-se do verdadeiro valor pela análise do percentual de variação, portanto, o viés aumentou para todos os métodos de estimação do modelo misto.

\subsubsection{Desempenho preditivo}

Os resultados da simulação do modelo misto e tradicional na base de ajuste (BA) e predição (BP) são discutidos nesta subseção. Para avaliar o desempenho preditivo da classificação da variável resposta, foram utilizadas as medidas AUC e KS, descritas no Capítulo 2.

$\mathrm{Na} \mathrm{BA}$ os efeitos aleatórios do modelo misto eram conhecidos, e assim, para avaliar a predição da probabilidade da resposta para este modelo, utilizou-se diretamente a função logito (4.1). Na 
Tabela 4.3: Média de AUC e KS para modelo tradicional e misto baseado em 1.000 réplicas, por método de estimação e predição, na base de ajuste e predição.

\begin{tabular}{|c|c|c|c|c|c|c|c|c|c|c|c|}
\hline \multirow{3}{*}{ Cenário } & \multirow{3}{*}{$\begin{array}{l}\text { Método de } \\
\text { Estimação }\end{array}$} & \multicolumn{2}{|c|}{ Base Ajuste } & \multicolumn{8}{|c|}{ Base de Predição por método de predição } \\
\hline & & \multirow{2}{*}{$\mathrm{AUC}$} & \multirow{2}{*}{ KS } & \multicolumn{4}{|c|}{ AUC } & \multicolumn{4}{|c|}{ KS } \\
\hline & & & & MZ & MPE & RL & NP & MZ & MPE & RL & NP \\
\hline \multirow{5}{*}{1} & Tradicional & $64,5 \%$ & $33,1 \%$ & \multicolumn{4}{|c|}{$63,5 \%$} & \multicolumn{4}{|c|}{$31,8 \%$} \\
\hline & QVP & $98,4 \%$ & $93,5 \%$ & $63,0 \%$ & $64,5 \%$ & $62,2 \%$ & $59,4 \%$ & $31,3 \%$ & $32,4 \%$ & $29,9 \%$ & $26,7 \%$ \\
\hline & $\mathrm{AL}$ & $98,7 \%$ & $92,4 \%$ & $63,5 \%$ & $64,3 \%$ & $63,0 \%$ & $60,8 \%$ & $31,8 \%$ & $32,2 \%$ & $30,8 \%$ & $28,1 \%$ \\
\hline & $\mathrm{QAGH}=2$ & $98,5 \%$ & $91,4 \%$ & $63,7 \%$ & $64,4 \%$ & $63,1 \%$ & $60,7 \%$ & $31,9 \%$ & $32,3 \%$ & $30,9 \%$ & $28,0 \%$ \\
\hline & $\mathrm{QAGH}=5$ & $98,5 \%$ & $91,2 \%$ & $63,7 \%$ & $64,4 \%$ & $63,2 \%$ & $60,8 \%$ & $32,0 \%$ & $32,4 \%$ & $30,9 \%$ & $28,0 \%$ \\
\hline \multirow{5}{*}{2} & Tradicional & $64,8 \%$ & $33,3 \%$ & \multicolumn{4}{|c|}{$62,3 \%$} & \multicolumn{4}{|c|}{$32,0 \%$} \\
\hline & QVP & $98,4 \%$ & $90,6 \%$ & $62,4 \%$ & $63,8 \%$ & $59,9 \%$ & $57,2 \%$ & $32,1 \%$ & $32,8 \%$ & $30,3 \%$ & $27,3 \%$ \\
\hline & $\mathrm{AL}$ & $98,2 \%$ & $89,8 \%$ & $62,5 \%$ & $63,8 \%$ & $60,4 \%$ & $57,7 \%$ & $32,1 \%$ & $32,8 \%$ & $30,5 \%$ & $27,8 \%$ \\
\hline & $\mathrm{QAGH}=2$ & $98,1 \%$ & $89,5 \%$ & $62,5 \%$ & $63,8 \%$ & $60,3 \%$ & $57,7 \%$ & $32,2 \%$ & $32,8 \%$ & $30,5 \%$ & $27,8 \%$ \\
\hline & $\mathrm{QAGH}=5$ & $98,0 \%$ & $89,4 \%$ & $62,5 \%$ & $63,8 \%$ & $60,4 \%$ & $57,7 \%$ & $32,2 \%$ & $32,9 \%$ & $30,5 \%$ & $27,8 \%$ \\
\hline \multirow{5}{*}{3} & Tradicional & $65,7 \%$ & $34,5 \%$ & \multicolumn{4}{|c|}{$60,8 \%$} & \multicolumn{4}{|c|}{$32,2 \%$} \\
\hline & QVP & $97,3 \%$ & $86,7 \%$ & $60,9 \%$ & $62,7 \%$ & $57,1 \%$ & $55,5 \%$ & $32,3 \%$ & $34,2 \%$ & $31,5 \%$ & $30,2 \%$ \\
\hline & $\mathrm{AL}$ & $97,0 \%$ & $86,1 \%$ & $61,0 \%$ & $62,4 \%$ & $57,6 \%$ & $55,7 \%$ & $32,3 \%$ & $33,9 \%$ & $31,9 \%$ & $30,1 \%$ \\
\hline & $\mathrm{QAGH}=2$ & $97,0 \%$ & $86,0 \%$ & $61,0 \%$ & $62,6 \%$ & $57,6 \%$ & $55,7 \%$ & $32,3 \%$ & $34,0 \%$ & $31,8 \%$ & $30,1 \%$ \\
\hline & $\mathrm{QAGH}=5$ & $97,0 \%$ & $86,0 \%$ & $60,9 \%$ & $62,5 \%$ & $57,6 \%$ & $55,7 \%$ & $32,3 \%$ & $33,9 \%$ & $31,8 \%$ & $30,1 \%$ \\
\hline \multirow{5}{*}{4} & Tradicional & $86,9 \%$ & $63,8 \%$ & \multicolumn{4}{|c|}{$90,5 \%$} & \multicolumn{4}{|c|}{$71,6 \%$} \\
\hline & QVP & $92,0 \%$ & $73,1 \%$ & $90,5 \%$ & $90,4 \%$ & $90,3 \%$ & $89,8 \%$ & $71,6 \%$ & $71,6 \%$ & $71,5 \%$ & $70,5 \%$ \\
\hline & $\mathrm{AL}$ & $89,9 \%$ & $68,9 \%$ & $90,5 \%$ & $90,5 \%$ & $90,4 \%$ & $90,2 \%$ & $71,6 \%$ & $71,6 \%$ & $71,6 \%$ & $71,2 \%$ \\
\hline & $\mathrm{QAGH}=2$ & $89,9 \%$ & $68,8 \%$ & $90,5 \%$ & $90,5 \%$ & $90,4 \%$ & $90,2 \%$ & $71,6 \%$ & $71,6 \%$ & $71,6 \%$ & $71,2 \%$ \\
\hline & $\mathrm{QAGH}=5$ & $89,9 \%$ & $68,8 \%$ & $90,5 \%$ & $90,5 \%$ & $90,4 \%$ & $90,2 \%$ & $71,6 \%$ & $71,6 \%$ & $71,6 \%$ & $71,2 \%$ \\
\hline \multirow{5}{*}{5} & Tradicional & $64,1 \%$ & $32,3 \%$ & \multicolumn{4}{|c|}{$61,7 \%$} & \multicolumn{4}{|c|}{$31,5 \%$} \\
\hline & QVP & $98,3 \%$ & $89,8 \%$ & $62,3 \%$ & $63,7 \%$ & $59,8 \%$ & $57,1 \%$ & $32,1 \%$ & $32,9 \%$ & $29,9 \%$ & $27,2 \%$ \\
\hline & $\mathrm{AL}$ & $98,0 \%$ & $88,9 \%$ & $62,8 \%$ & $63,3 \%$ & $60,5 \%$ & $58,0 \%$ & $32,5 \%$ & $32,6 \%$ & $30,6 \%$ & $28,0 \%$ \\
\hline & $\mathrm{QAGH}=2$ & $97,9 \%$ & $88,7 \%$ & $62,7 \%$ & $63,3 \%$ & $60,5 \%$ & $58,0 \%$ & $32,4 \%$ & $32,7 \%$ & $30,5 \%$ & $27,9 \%$ \\
\hline & $\mathrm{QAGH}=5$ & $97,9 \%$ & $88,6 \%$ & $62,7 \%$ & $63,3 \%$ & $60,5 \%$ & $58,0 \%$ & $32,4 \%$ & $32,7 \%$ & $30,5 \%$ & $28,0 \%$ \\
\hline \multirow{5}{*}{6} & Tradicional & $67,1 \%$ & $36,9 \%$ & & 66 , & $5 \%$ & & & & $\%$ & \\
\hline & QVP & $98,1 \%$ & $89,7 \%$ & $67,2 \%$ & $67,2 \%$ & $63,6 \%$ & $59,8 \%$ & $37,8 \%$ & $37,6 \%$ & $34,2 \%$ & $30,5 \%$ \\
\hline & $\mathrm{AL}$ & $97,7 \%$ & $88,1 \%$ & $67,3 \%$ & $67,3 \%$ & $64,1 \%$ & $60,5 \%$ & $37,9 \%$ & $37,6 \%$ & $34,9 \%$ & $31,4 \%$ \\
\hline & $\mathrm{QAGH}=2$ & $97,6 \%$ & $87,9 \%$ & $67,3 \%$ & $67,3 \%$ & $64,1 \%$ & $60,5 \%$ & $37,9 \%$ & $37,7 \%$ & $34,9 \%$ & $31,4 \%$ \\
\hline & $\mathrm{QAGH}=5$ & $97,6 \%$ & $87,7 \%$ & $67,4 \%$ & $67,3 \%$ & $64,1 \%$ & $60,5 \%$ & $38,0 \%$ & $37,7 \%$ & $34,8 \%$ & $31,4 \%$ \\
\hline & Tradicional & $59,4 \%$ & $26,2 \%$ & & & $5 \%$ & & & & $\%$ & \\
\hline & QVP & $98,4 \%$ & $90,3 \%$ & $51,9 \%$ & $53,7 \%$ & $51,2 \%$ & $50,9 \%$ & $19,0 \%$ & $20,7 \%$ & $20,5 \%$ & $21,0 \%$ \\
\hline 7 & $\mathrm{AL}$ & $98,0 \%$ & $88,9 \%$ & $52,0 \%$ & $53,4 \%$ & $51,0 \%$ & $50,8 \%$ & $19,2 \%$ & $20,6 \%$ & $20,1 \%$ & $21,0 \%$ \\
\hline & $\mathrm{QAGH}=2$ & $97,9 \%$ & $88,6 \%$ & $51,9 \%$ & $53,4 \%$ & $51,0 \%$ & $50,9 \%$ & $19,0 \%$ & $20,6 \%$ & $20,1 \%$ & $21,1 \%$ \\
\hline & $\mathrm{QAGH}=5$ & $97,8 \%$ & $88,5 \%$ & $51,7 \%$ & $53,4 \%$ & $51,1 \%$ & $50,9 \%$ & $18,9 \%$ & $20,7 \%$ & $20,1 \%$ & $21,0 \%$ \\
\hline
\end{tabular}

BP, na qual não se conhecia o valor individual dos efeitos aleatórios para os novos grupos, foram utilizados os métodos de predição: média zero (MZ), melhor preditor empírico (MPE), regressão linear (RL) e não-paramétrico (NP), descritos no Capítulo 3.

Para o modelo tradicional, na BA e BP, a predição da probabilidade foi realizada utilizando a função logito (4.2), para se fazer a predição da resposta.

A Tabela 4.3 apresenta os valores médios de AUC e KS baseados nas 1.000 réplicas, na BA e BP, para os sete cenários descritos na Tabela 4.1. A Tabela 4.3 foi colorida de tal forma que as tonalidades mais próximas de verde indicam maiores valores médios de AUC e KS, consequentemente melhores desempenhos dentro do cenário. Por outro lado, tonalidades próxima de vermelho, 
indicam os piores desempenhos.

Analisando esta tabela, na BA, a média de AUC e KS dos métodos de estimação do modelo misto indicou um nível de predição muito superior ao modelo tradicional, para todos os cenários. No geral, o desempenho da predição da resposta para o modelo misto foi superior para o método de estimação QVP, seguido por AL e posteriormente pela QAGH. Nota-se que a QAGH para 2 e 5 pontos apresentaram valores praticamente iguais de AUC e KS.

$\mathrm{Na} \mathrm{BP}$, a diferença de desempenho do modelo tradicional para o misto foi menor do que na BA. Este resultado era esperado, já que não se conhecia os valores dos efeitos aleatórios para novos grupos. Analisando as medidas de desempenho da BP, para o modelo misto não se observaram praticamente diferenças entre os métodos de estimação, a exceção ocorreu apenas para QVP.

Por outro lado, observaram-se diferenças expressivas entre os métodos de predição, pela análise dos valores médios de AUC e KS, no qual, de maneira geral, o método MPE apresentou desempenho preditivo superior aos demais métodos de predição, seguido pelo método MZ, tradicional, RL e NP.

Mais detalhes sobre o desempenho dos métodos de predição pelas medidas AUC e KS, na BA e BP, são fornecidos a seguir, por estudo de simulação.

\section{Estudo 1 - Resultados da variação do tamanho do grupo (cenários: 1, 2 e 3)}

Os resultados indicaram que quando o tamanho do grupo $(n)$ aumentou de 3 para 12:

- Base de ajuste:

- A média do AUC manteve-se entre $97 \%$ e $99 \%$ para o modelo misto, e para o modelo tradicional entre $64 \%$ e $66 \%$.

- A média do KS diminuiu levemente apenas para os métodos de predição do modelo misto, mantendo-se superior a $86 \%$, e para o caso do modelo tradicional mantendo-se superior a $33 \%$.

- Base de predição:

- A média de AUC diminuiu um pouco para todos os métodos de estimação e predição, de aproximadamente $65 \%$ para $55 \%$. O método MPE foi aquele que apresentou melhor desempenho, seguido pelo MZ, RL e NP. Os métodos MPE e MZ apresentaram poder preditivo superior ao tradicional (maiores valores com tonalidade verde), enquanto RL e NP apresentaram desempenho inferior ao tradicional (menores valores com tonalidade vermelha).

- A média de KS aumentou levemente para todos os métodos de estimação e predição, de aproximadamente $26 \%$ para $34 \%$. O desempenho preditivo dos métodos MPE, RL e NP melhoram em relação ao modelo tradicional à medida que o tamanho de grupo aumentou, com MPE apresentando desempenho superior ao modelo tradicional. O método MZ não apresentou praticamente diferença à medida que o tamanho do grupo aumentou. 
Nota-se na BP que não existiu um mesmo padrão de comportamento para os índices KS e AUC em relação ao aumento de tamanho de grupo.

\section{Estudo 2 -Resultados da variação de $\sigma_{1}$ e $\sigma_{2}$ (cenários: 4 e 2)}

Os resultados indicaram que quando o valor do desvio-padrão dos efeitos aleatórios $\left[\sigma_{1} ; \sigma_{2}\right]$ aumentou de $[0,5 ; 0,5]$ para $[5,0 ; 5,0]$ :

- Base de ajuste:

- As médias de AUC e KS aumentaram para os métodos de estimação do modelo misto. O AUC (KS) apresentava média de aproximadamente $90 \%$ (69\%) no cenário 4, enquanto no cenário 2, o valor foi de aproximadamente $98 \%$ (90\%). No caso do modelo tradicional, ocorreu o contrário, pois o AUC (KS) apresentava média de aproximadamente 87\% (65\%) no cenário 4, enquanto no cenário 2, o valor foi de aproximadamente $64 \%$ (33\%).

- Base de predição:

- As médias de AUC e KS diminuíram expressivamente. Por exemplo, para a medida AUC, no cenário 4 os valores médios estavam próximos a 90\%, enquanto no cenário 2, os valores foram próximos a $60 \%$. No caso do KS, a média estava num patamar de $70 \%$ no cenário 4, e caiu para aproximadamente $30 \%$ no cenário 2 . A predição do método MPE apresentou desempenho um pouco superior em relação ao modelo tradicional à medida que $\left[\sigma_{1} ; \sigma_{2}\right]$ aumentaram. O método MZ não apresentou diferença no desempenho preditivo, e o desempenho preditivo dos métodos RL e NP pioraram em relação ao modelo tradicional. Observa-se na BP no cenário 2 que os métodos MPE e MZ apresentam nível preditivo superior em relação aos demais métodos de predição. Além disso, no cenário 4, todos os métodos apresentaram desempenho preditivo similares, inclusive RL e NP, que geralmente apresentaram poder preditivo inferior em relação aos outros métodos.

Logo, pode-se concluir que a medida que os valores dos desvios-padrão dos efeitos aletatórios aumenta, melhor é o desempenho dos métodos de predição do modelo misto.

\section{Estudo 3 -Resultados da variação de $\sigma_{12}$ (cenários: 5, 2 e 6)}

Os resultados indicaram que quando os valores $\left[\sigma_{12} ; \rho\right]$ aumentaram de $[3,750 ; 0,15]$ para $[21,125 ; 0,85]$ :

- Base de ajuste:

- A média do AUC (KS) ficou no mesmo patamar de predição, aproximadamente 98\% (90\%), para os métodos de predição do modelo misto. No caso do modelo tradicional, observou-se um leve aumento na média de AUC (KS) de 64\% (32\%) para 67\% (37\%).

- Base de predição: 
- A média do AUC (KS) aumentou de 57\% (27\%) para 67\% (38\%). O desempenho preditivo do método MPE, RL e NP pioraram em relação ao modelo tradicional.

Logo, os métodos de predição do modelo misto parecem funcionar melhor para valores menores de $\sigma_{12}$. Com exceção do método MZ que parece funcionar melhor quando $\sigma_{12}$ aumenta. Este resultado é coerente, uma vez que a alta correlação entre os efeitos aleatórios é um indicativo de que um dos efeitos aleatórios não precisaria estar na parte aleatória do modelo.

\section{Estudo 4 - Resultados da variação de $\beta$ (cenários: 7 e 2)}

Os resultados indicaram que quando o valor $\beta$ aumentou:

- Base de ajuste:

- A média de AUC (KS) aumentou para o modelo tradicional, de 59\% (26\%) para 65\% $(33 \%)$. Os demais métodos de estimação mantiveram o mesmo patamar de predição de AUC (KS), com valores aproximados de $98 \%$ (90\%).

- Base de predição:

- A média do AUC aumentou de $51 \%$ para $62 \%$, aproximadamente. O desempenho da predição de todos os métodos de predição piorou em relação ao modelo tradicional, ou seja, os métodos de predição para o modelo misto parecem funcionar melhor para o cenário 7, sendo o método MPE o mais eficiente, seguido pelo NP, RL, MZ e por último o tradicional.

- A média do KS aumentou de $18 \%$ para 33\%, aproximadamente. As mesmas conclusões do item anterior, porém no cenário 7, MPE e NP apresentaram desempenhos superiores aos demais métodos, seguido por RL, MZ e tradicional.

A Tabela 4.3 apresentou a média dos valores de AUC e KS, baseada nas 1.000 réplicas da simulação. Para mais detalhes, no Apêndice A são fornecidos os gráficos do tipo boxplot para estas duas medidas de desempenho em: A.1, A.2, A.3, A.4, A.5, A.6 e A.7, referentes aos respectivos cenários de simulação. Pela análise dos boxplots, as conclusões para AUC e KS foram as mesmas obtidas pela análise da média apresentada anteriormente.

\subsubsection{Resíduos dos valores preditos}

Na subseção anterior, foi avaliado o poder preditivo em termos de classificação da resposta pelas medidas AUC e KS. Nesta subseção, é apresentada a avaliação dos resíduos da predição, descritas na introdução deste capítulo, que traz uma perspectiva de análise baseada na comparação entre as probabilidades predita e verdadeira de uma dada observação.

Analisando os valores Md.res.qua e Md.res.abs apresentados na Tabela (4.4), observou-se de maneira geral, que o patamar do valor da mediana dos resíduos são muito próximos para os cenários 
Tabela 4.4: Md.res.qua e Md.res.abs na BP, por método de estimação e predição.

\begin{tabular}{|c|c|c|c|c|c|c|c|c|c|}
\hline \multirow{3}{*}{ Cenário } & \multirow{3}{*}{$\begin{array}{l}\text { Método de } \\
\text { Estimação }\end{array}$} & \multicolumn{8}{|c|}{ Resíduos avaliados na Base de Predição por método de predição } \\
\hline & & \multicolumn{4}{|c|}{ Md.res.qua } & \multicolumn{4}{|c|}{ Md.res.abs } \\
\hline & & MZ & MPE & RL & NP & MZ & MPE & RL & NP \\
\hline \multirow{5}{*}{1} & Tradicional & \multicolumn{4}{|c|}{0,168} & \multicolumn{4}{|c|}{0,410} \\
\hline & QVP & 0,117 & 0,099 & 0,121 & 0,125 & 0,342 & 0,314 & 0,348 & 0,353 \\
\hline & $\mathrm{AL}$ & 0,101 & 0,084 & 0,111 & 0,112 & 0,318 & 0,290 & 0,332 & 0,334 \\
\hline & $\mathrm{QAGH}=2$ & 0,110 & 0,090 & 0,116 & 0,118 & 0,331 & 0,300 & 0,341 & 0,343 \\
\hline & $\mathrm{QAGH}=5$ & 0,111 & 0,093 & 0,117 & 0,118 & 0,333 & 0,305 & 0,341 & 0,344 \\
\hline \multirow{5}{*}{2} & Tradicional & \multicolumn{4}{|c|}{0,160} & \multicolumn{4}{|c|}{0,400} \\
\hline & QVP & 0,091 & 0,088 & 0,110 & 0,116 & 0,301 & 0,296 & 0,332 & 0,340 \\
\hline & $\mathrm{AL}$ & 0,087 & 0,082 & 0,108 & 0,112 & 0,295 & 0,286 & 0,327 & 0,334 \\
\hline & $\mathrm{QAGH}=2$ & 0,090 & 0,085 & 0,110 & 0,111 & 0,301 & 0,292 & 0,331 & 0,334 \\
\hline & $\mathrm{QAGH}=5$ & 0,093 & 0,085 & 0,110 & 0,112 & 0,304 & 0,292 & 0,332 & 0,334 \\
\hline \multirow{5}{*}{2} & Tradicional & \multicolumn{4}{|c|}{0,168} & \multicolumn{4}{|c|}{0,409} \\
\hline & QVP & 0,118 & 0,107 & 0,119 & 0,135 & 0,344 & 0,327 & 0,344 & 0,367 \\
\hline & $\mathrm{AL}$ & 0,116 & 0,105 & 0,116 & 0,129 & 0,340 & 0,324 & 0,341 & 0,359 \\
\hline & QAGH=2 & 0,119 & 0,107 & 0,117 & 0,129 & 0,344 & 0,327 & 0,341 & 0,358 \\
\hline & $\mathrm{QAGH}=5$ & 0,118 & 0,108 & 0,116 & 0,130 & 0,343 & 0,328 & 0,341 & 0,358 \\
\hline \multirow{5}{*}{4} & Tradicional & \multicolumn{4}{|c|}{0,002} & \multicolumn{4}{|c|}{0,045} \\
\hline & QVP & 0,002 & 0,003 & 0,002 & 0,003 & 0,043 & 0,050 & 0,047 & 0,053 \\
\hline & $\mathrm{AL}$ & 0,002 & 0,002 & 0,002 & 0,002 & 0,042 & 0,045 & 0,043 & 0,045 \\
\hline & $\mathrm{QAGH}=2$ & 0,002 & 0,002 & 0,002 & 0,002 & 0,042 & 0,045 & 0,043 & 0,045 \\
\hline & $\mathrm{QAGH}=5$ & 0,002 & 0,002 & 0,002 & 0,002 & 0,042 & 0,045 & 0,043 & 0,045 \\
\hline \multirow{5}{*}{5} & Tradicional & \multicolumn{4}{|c|}{0,167} & \multicolumn{4}{|c|}{0,408} \\
\hline & QVP & 0,106 & 0,100 & 0,115 & 0,122 & 0,325 & 0,316 & 0,340 & 0,350 \\
\hline & $\mathrm{AL}$ & 0,098 & 0,095 & 0,115 & 0,121 & 0,312 & 0,309 & 0,338 & 0,348 \\
\hline & $\mathrm{QAGH}=2$ & 0,102 & 0,097 & 0,115 & 0,121 & 0,319 & 0,312 & 0,339 & 0,347 \\
\hline & $\mathrm{QAGH}=5$ & 0,103 & 0,099 & 0,116 & 0,122 & 0,321 & 0,315 & 0,341 & 0,348 \\
\hline \multirow{5}{*}{6} & Tradicional & \multicolumn{4}{|c|}{0,142} & \multicolumn{4}{|c|}{0,376} \\
\hline & QVP & 0,066 & 0,060 & 0,083 & 0,098 & 0,257 & 0,243 & 0,287 & 0,312 \\
\hline & $\mathrm{AL}$ & 0,066 & 0,058 & 0,085 & 0,092 & 0,258 & 0,241 & 0,291 & 0,303 \\
\hline & $\mathrm{QAGH}=2$ & 0,068 & 0,061 & 0,086 & 0,093 & 0,259 & 0,246 & 0,293 & 0,305 \\
\hline & $\mathrm{QAGH}=5$ & 0,069 & 0,062 & 0,087 & 0,094 & 0,263 & 0,248 & 0,294 & 0,306 \\
\hline \multirow{5}{*}{7} & Tradicional & \multicolumn{4}{|c|}{0,198} & & & & \\
\hline & QVP & 0,170 & 0,140 & 0,164 & 0,167 & 0,412 & 0,374 & 0,404 & 0,409 \\
\hline & $\mathrm{AL}$ & 0,165 & 0,138 & 0,162 & 0,165 & 0,407 & 0,371 & 0,402 & 0,406 \\
\hline & $\mathrm{QAGH}=2$ & 0,167 & 0,140 & 0,162 & 0,164 & 0,408 & 0,374 & 0,403 & 0,404 \\
\hline & $\mathrm{QAGH}=5$ & 0,168 & 0,140 & 0,163 & 0,162 & 0,410 & 0,374 & 0,403 & 0,403 \\
\hline
\end{tabular}

1, 2, 3, 5 e 6, para todos os métodos de predição. O modelo tradicional foi aquele que apresentou maior resíduo comparado aos métodos de predição do modelo misto, como esperado. Comparando os métodos de predição para o modelo misto, os mesmos apresentaram valores dos resíduos no mesmo patamar, sendo o MPE o método que apresentou menor mediana dos resíduos, seguido pelos métodos MZ, RL e NP.

Para os cenários 4 e 7, os valores dos resíduos foram diferentes dos cenários descritos no parágrafo anterior. Uma característica comum entre estes dois cenários é que os valores da mediana dos resíduos dos métodos de predição do modelo misto ficaram mais próximos dos resíduos do modelo tradicional. Os resultados são coerentes, pois nestes dois cenários atribuiu-se um peso menor à 
parte aleatória no preditor linear do modelo misto. Neste caso, os modelos misto e tradicional apresentam "maior similaridade", e por isso, era esperado que os resíduos do modelo misto e tradicional apresentassem valores mais próximos.

Na Tabela (4.4) utilizou-se a mediana dos resíduos para fazer a avaliação dos cenários simulados. Para mais detalhes, no Apêndice A são fornecidos os gráficos do tipo boxplot dos resíduos ao quadrado e resíduos absolutos, para cada cenários de simulação em: A.1, A.2, A.3, A.4, A.5, A.6 e A.7. As conclusões obtidas pela análise dos boxplots foram as mesmas das medidas Md.res.qua e Md.res.abs.

\subsubsection{Discussão do uso dos métodos de predição}

Em resumo, as seguintes conclusões práticas podem ser obtidas para os métodos de predição de novos grupos, avaliadas na BP, sob o ponto de vista de discriminação da resposta (AUC e KS) e resíduos da predição (Md.res.qua e Md.res.abs):

- O método de predição MPE apresentou, no geral, o melhor desempenho na predição da resposta em relação ao modelo tradicional e em relação à todos os demais métodos de predição para o modelo misto.

- O método MZ, no geral, mostrou-se o mais eficiente em termos de predição depois do MPE; com exceção do cenário 7, quando o valor do $\beta=1$, para a medida KS. Este resultado é coerente, pois neste cenário diminuiu o peso da parte fixa no preditor linear modelo misto, e como os métodos tradicional e MZ consideram somente a parte fixa do modelo, a predição destes métodos ficaram prejudicadas. Também verificou-se no cenário 6 que à medida que $\sigma_{12}$ aumenta, o método MZ parece funcionar melhor, pois anular a parte aleatória do modelo parece sofrer menos influência para maiores valores de $\rho$.

- O método RL e NP pareceram ser relevantes quando a parte fixa do modelo tem um peso menor com alta variabilidade dos efeitos aleatórios, como apresentado no cenário 7 . Inclusive neste cenário, NP apresentou predição de KS nos mesmos patamares de RL e MPE.

- O método NP especificamente parece precisar de estudos mais aprofundados em relação ao método RL, pois no geral este foi o método que apresentou poder preditivo mais fraco em relação aos outros métodos. Possivelmente, esse resultado ocorreu devido a imposição dos efeitos aleatórios verdadeiros partirem de uma normal, dado o modelo verdadeiro (4.1). Mesmo no cenário 7, no qual a medida KS apresentou melhor desempenho dentre os demais métodos, para AUC, Md.res.qua e Md.res.abs este foi o método que apresentou pior desempenho.

- Em relação aos resíduos da predição, no geral, o mesmo padrão para os distintos cenários apresentados manteve-se do melhor para o pior método de predição: MPE, MZ, RL, NP e tradicional. 
- A importância dos métodos de predição aparentemente não está associada fortemente ao viés das estimativas dos parâmetros $\beta, \sigma_{1}, \sigma_{2}$ e $\sigma_{12}$, pois mesmo em alguns cenários nos quais foi verificado menor viés nas estimativas dos parâmetros, os resultados da predição foram similares àqueles que o viés foi maior. Inclusive, observou-se que os métodos de estimação AL e QAGH para 2 e 5 pontos na quadratura apresentaram predição da resposta bem similares.

\subsection{Aspectos Computacionais}

Nesta seção são discutidas as questões computacionais envolvidas nos estudos de simulação, tais como detalhes sobre os comandos utilizados no R 2.10.1 para rodar o código de simulação, problemas na estimação dos parâmetros, e complexidade computacional para os diversos métodos de estimação e predição.

Os métodos de estimação (QVP, AL e QAGH) e predição (MZ, MPE, RL e NP) foram programados no software R, versão 2.10.1, Team (2010).

O modelo logístico misto foi ajustado pelos métodos de estimação: QVP, AL, QAGH com 2 e 5 pontos na quadratura. No R, os seguintes comandos foram utilizados: glmmPQL para método QVP e glmer para os métodos AL e QAGH. A configuração padrão do glmer considera 1 ponto na quadratura na QAGH, que é equivalente ao método da AL, como demonstrado no Capítulo 3. Para QAGH, ao utilizar-se o comando glmer é necessário inserir dentro da rotina "nAGQ=pontos", com "pontos"sendo a quantidade de pontos na quadratura desejada.

O modelo logístico tradicional foi ajustado utilizando o comando glm, que faz a estimação dos parâmetros por máxima verossimilhança usando o algoritmo de Escore de Fisher. Para a predição, nos casos do modelo tradicional e do método MZ, não foi necessário nenhum comando específico, pois utilizou-se a função logito que foi facilmente implementada.

O método de predição MPE requereu o cálculo de integrais multidimensionais, conforme (3.44). Para este fim, foi usado o comando divonne, que funciona com amostragem estratificada, em que o particionamento é auxiliado por métodos de otimização numérica. Esta função está disponível na biblioteca R2Cuba. A documentação para o usuário está disponível em Hahn (2005). Outros exemplos de comandos para calcular integrais multidimensionais dentro da mesma biblioteca são cuhre, suave e vegas. Todos estes comandos foram testados e decidiu-se usar o divonne porque foi o mais rápido e resultou em valores semelhantes aos dos outros disponíveis.

Para os métodos RL e NP, utilizou-se, respectivamente, as rotinas $\mathbf{l m}$ e gam. No caso do NP, a função está disponível na biblioteca gam de Hastie e Tibshirani (1990). Para se fazer a seleção de modelos, foram utilizados os comandos stepAIC para o método RL e step.gam para o método NP. A biblioteca gam permite que a função de suavização seja definida pelo usuário para os casos: smoothing splines representada por $s$ (covariável) e loess smooth terms representada por lo(covariável). 


\subsubsection{Custo computacional dos métodos de estimação e predição}

Nesta subseção é discutido o custo computacional, em termos de complexidade computacional das rotinas utilizadas, para os métodos de estimação e predição considerados nos estudos de simulação.

Na etapa de estimação dos parâmetros, observou-se que a quantidade de pontos na quadratura para o método QAGH aumentou substancialmente o tempo computacional na obtenção das estimativas dos parâmetros. Essa questão computacional do método QAGH em relação aos pontos na quadratura foram discutidas também por Liu e Pierce (1994) e Rabe-Hesketh et al. (2002).

A complexidade computacional de todos os métodos de estimação também está relacionada ao tamanho da base de dados. No caso dos bancos de dados que consideram uma quantidade grande de observações e variáveis preditoras, os métodos de estimação requerem um tempo de processamento maior, e nos casos mais extremos, não executam a rotina.

A Tabela 4.5 apresenta a contagem associada aos problemas ocorridos durante a estimação dos parâmetros, por método de estimação e por cenário, para conseguir-se completar as 1.000 réplicas requeridas. Foram contabilizados como problemas: falta de convergência na estimativa dos parâmetros e estimativas das componentes de variância com pelo menos um dos valores sendo igual a zero, infinito ou nulo.

Por exemplo, no cenário 1 foram necessárias 732 réplicas adicionais para se completar as 1.000 réplicas desejadas, o que indica que os métodos de estimação podem ser ainda melhorados por apresentarem muitos problemas na estimação dos parâmetros. QVP foi o método que apresentou maior quantidade de ocorrências comparado aos demais métodos de estimação em todos os cenários. Em especial, no cenário 4, observou-se uma grande quantidade de ocorrências, necessitando de mais de 17.000 bancos até completar as 1.000 réplicas.

Tabela 4.5: Número de problemas ocorridos na estimação dos parâmetros do modelo misto até se completar as 1.000 réplicas desejadas.

\begin{tabular}{lccccccc}
\hline \multirow{2}{*}{ Método de Estimação } & \multicolumn{7}{c}{ Cenário } \\
\cline { 2 - 8 } & 1 & 2 & 3 & 4 & 5 & 6 & 7 \\
\hline $\mathrm{QVP}$ & 618 & 501 & 722 & 16.665 & 828 & 794 & 1.114 \\
$\mathrm{AL}$ & 107 & 73 & 71 & 713 & 213 & 58 & 72 \\
$\mathrm{QHG}=2$ & 6 & 1 & 2 & 334 & 1 & 1 & 2 \\
$\mathrm{QGH}=5$ & 1 & 0 & 0 & 147 & 0 & 1 & 0 \\
\hline \multicolumn{1}{c}{ Total } & 732 & 575 & 795 & 17.859 & 1.042 & 854 & 1.188 \\
\hline
\end{tabular}

Na etapa de predição pelo método MPE, baseado na equação (3.48), o tempo de execução aumentou de forma linear em relação ao tamanho do grupo. Isso ocorreu, pois à medida que o tamanho do grupo aumentou, aumentou também a quantidade de elementos dentro do integrando da integral multidimensional. Os demais métodos de predição, MZ, RL e NP, apresentaram pouquíssimo esforço computacional no tempo de execução de suas rotinas. Por exemplo, o método MPE demorou, em média, 60 segundos para calcular o valor predito por observação, enquanto os demais métodos demoraram menos de 1 segundo. 
O código de simulação para uma réplica contemplou todos os métodos de estimação e predição simultaneamente. O tempo de execução deste código considerando as 1.000 réplicas foi custoso, uma vez que esse tempo foi função da quantidade de réplicas, da quantidade de problemas na estimação dos parâmetros e do método MPE. Para simular um determinado cenário, o tempo médio foi de aproximadamente 15 dias, utilizando um computador com maior poder de processamento (disponível nos servidores do IME-USP). Caso esse mesmo cenário de simulação fosse processado em um computador comum, seria necessário quase o dobro do tempo.

Dentre os métodos de estimação para o modelo logístico misto, na prática, a AL é mais a indicada por apresentar menor viés e uma menor quantidade de problemas na estimação dos parâmetros, conforme Tabela 4.5, embora tenha apresentado um pouco mais de problemas na estimação dos parâmetros comparado a QAGH.

Em relação aos métodos de predição, na prática, MPE é viável para banco de dados e tamanhos de grupos não muito grandes. Por exemplo, banco de dados com quantidade inferior a 50 mil registros e tamanho de grupo inferior a 500. Quando o banco de dados é muito grande, é recomendado utilizar os demais métodos de predição, embora eles tenham apresentado, no geral, desempenhos inferiores ao método MPE. 


\section{Capítulo 5}

\section{Aplicações}

Devido à sua importância, modelos preditivos têm sido utilizados em diversos campos de aplicação, tais como medicina, ciências sociais, finanças, marketing, entre outros.

Dentro do campo da medicina, áreas como a da saúde pública têm sido beneficiadas das informações disponibilizadas por estes modelos. Na prática clínica, modelos para predizer futuras ocorrências têm sido extensivamente usados na tomada de decisão após o diagnóstico do paciente ter sido efetuado. Baseado, por exemplo, na probabilidade do paciente apresentar a doença, a decisão pode incidir na necessidade de exames adicionais ou na forma de tratamentos preventivos da doença. Também tem se tornado importante a necessidade de prever a incidência de determinadas doenças considerando a evolução temporal. Assim, novas metodologias estatísticas foram propostas, e os modelos mistos, por exemplo, podem ser utilizados para predizer a probabilidade do indivíduo apresentar a doença ao logo do tempo. Papoila (2012) discute a importância dos modelos de predição na área da medicina, e cita alguns trabalhos na literatura para avaliar o desempenho dos modelos preditivos, como os artigos de Cook (2007), Pencina et al. (2008) e Steyerberg et al. (2010).

No campo do marketing, modelos preditivos têm sido usados em estratégias de CRM (customer relationship managment), que focam no relacionamento do cliente com a empresa por meio de planejamento de campanhas de marketing. As estratégias de CRM vão desde a conquista de um novo cliente, rentabilização do mesmo dentro da empresa, até a prevenção da saída dele. A seleção do público-alvo a ser abordado nas campanhas de marketing é realizada com base nas probabilidades ou scores dos clientes apresentarem a resposta de interesse gerada a partir de modelos preditivos. Kamakura et al. (2003) utilizaram a propensão de compra para a venda de novos produtos e serviços, baseado nas transações dos clientes dentro da empresa. Rosset et al. (2001) discutiram a importância dos modelos preditivos pela avaliação e comparação destes modelos nas campanhas de marketing, concentrando a atenção em critérios de avaliação dos modelos. Apesar da predição ser fundamental para as estratégias de abordagem do cliente, no contexto de predição de dados futuros utilizando modelos mistos, a literatura ainda não apresentou trabalhos relacionados a este tema.

Neste capítulo, são apresentadas duas aplicações dos métodos de predição do modelo logístico misto utilizando bancos de dados reais. Cada estudo de aplicação provêm de duas áreas distintas, sendo uma na área da saúde e outra na área de marketing. 
O primeiro banco de dados, proveniente da área da saúde pública, considera dados longitudinais ou dados com medidas repetidas, no qual se tem o interesse de avaliar as condições nutricionais de bebês, desde o nascimento até os 18 meses de vida. Nesta aplicação, a estrutura hierárquica é considerada da seguinte forma: as crianças são os grupos e suas medidas nutricionais ao longo do tempo são as observações dentro do grupo. Uma vez que estudos longitudinais utilizam amostras para inferir o comportamento populacional, selecionou-se parte do banco de dados para ajustar o modelo, e o restante para fazer a predição, simulando uma aplicação do modelo em um período futuro. Desta forma, quando surgir um novo recém-nascido, pode-se predizer sua probabilidade de apresentar desnutrição grave nos seus respectivos tempo futuros.

O segundo banco de dados, proveniente da área da marketing, considera as informações de uma empresa de telefonia móvel, no qual se tem o interesse de identificar quais os perfis dos funcionários que adquirem o pacote de mensagens (SMS - short message service). Neste caso, a estrutura hierárquica é dada da seguinte maneira: os clientes corporativos (empresas) são os grupos e as linhas de celular concedidas aos funcionários para fins comerciais são as unidades dentro do grupo. Neste tipo de aplicação, geralmente, a empresa coleta os dados de todos os clientes, portanto, temse o universo das unidades amostrais. Porém, para a predição da resposta em um período futuro, é necessário ter o modelo ajustado em um período anterior, para que possa ser atribuído a um funcionário, pertencente a uma nova empresa, a probabilidade de compra do produto.

Neste capítulo, são apresentados os resultados provenientes do método de estimação Aproximação de Laplace (AL). Os resultados da QAGH foram omitidos, pois apresentaram resultados similares à AL, e o método QVP não convergiu nas estimativas dos parâmetros para os dois bancos de dados. Esse mesmo comportamento em relação aos métodos de estimação foi observado nos estudos de simulação, conforme discutido no capítulo anterior.

Como o foco principal deste trabalho é fazer a predição para novos grupos, a aplicação de todos os métodos de predição propostos é apresentada neste capítulo, sendo eles: MPE (melhor preditor empírico), RL (regressão linear), NP (não-paramétrico) e MZ (média zero). Como análise complementar, foi ajustado também o modelo logístico tradicional por ser uma opção simples para se fazer a predição da resposta, dado que o modelo não considera a presença dos efeitos aleatórios.

Em ambas as aplicações têm-se o interesse de predizer a resposta para observações provenientes de novos grupos. Desta forma, o objetivo de cada aplicação é avaliar o desempenho da predição dos modelos obtidos pelos métodos de predição aplicados em um período futuro, ou seja, avaliando o desempenho preditivo do modelo em observações provenientes de grupos que não foram utilizados no ajuste do modelo.

Para avaliar o desempenho em termos de predição, foram usadas as medidas de desempenho estatística de Kolmogorov-Smirnov (KS) e Area Under the Curve (AUC), conforme descrito no Capítulo 2. Nota-se que no caso do uso de banco de dados reais, não se conhece o valor da probabilidade verdadeira, e por isso não foi possível fazer a análise dos resíduos da predição, como realizado nos estudos de simulação apresentados no Capítulo 4. 


\subsection{Aplicação em Dados Longitudinais: Modelo para avaliar desnutrição grave}

A fim de avaliar as condições nutricionais de crianças recém-nascidas, foi considerada uma base de dados longitudinais com 241 recém-nascidos do sexo masculino. As informações de cada criança foram observadas nos períodos de 2, 4, 6, 9, 12, 15 e 18 meses após o nascimento. No contexto dos modelos mistos, a criança $i$ foi considerada como grupo, com $j$ observações ao longo do tempo $(j=1, \ldots, 7)$, vide Figura 5.1

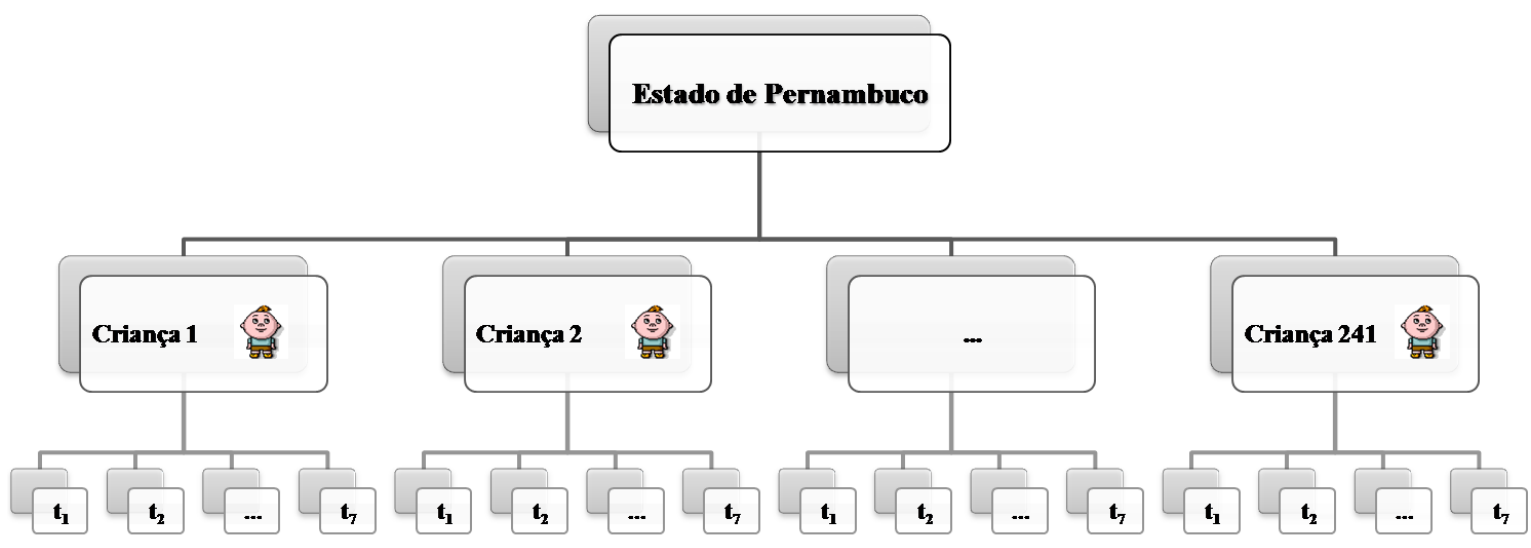

Figura 5.1: Estrutura hierárquica do banco de dados de desnutrição.

O escore HAZ é uma classificação do estado nutricional, com base na altura da criança, coletada em cada um dos 7 períodos específicos de observação. A variável resposta do problema é o escore HAZ, classificado em duas categorias:

- 1 - desnutrição grave;

- 0 - caso contrário.

O peso da criança foi a informação utilizada como covariável para explicar o escore HAZ. Ela foi coletada no momento do nascimento e nos 7 períodos de observação ao longo do tempo.

A estrutura do banco de dados pode ser visualizada na Figura 5.2.

Nesta aplicação, é apresentado o uso dos métodos de predição considerando um modelo com estrutura similar aos cenários dos estudos de simulação, no sentido de considerar a mesma covariável na parte fixa e aleatória do modelo. A estratégia de considerar a mesma covariável na parte fixa e aleatória no modelo tem sido sugerida na literatura para que os efeitos aleatórios não apresentem alta correlação. Baayen et al. (2008) discutiram que a alta correlação entre os efeitos aleatórios podem indicar que o modelo tenha sido superparametrizado, não sendo necessária a existência de algum dos efeitos aleatórios. Nos estudos de aplicação, não se pretende entrar em detalhes na seleção de modelos, pois o objetivo da aplicação é ilustrar o uso dos métodos de predição, dado um determinado modelo previamente selecionado.

Para ilustrar o procedimento, considerou-se uma amostra aleatória de $50 \%$ dos grupos na base de ajuste (BA). O restante dos grupos foi considerado na base de predição (BP), conforme Figura 5.3. 


\begin{tabular}{cccc}
\hline criança & tempo de observação & escore HAZ & peso(kg) \\
\hline 1 & 1 & 1 & 5,3 \\
1 & 2 & 0 & 7,8 \\
1 & 3 & 1 & 8,3 \\
1 & 4 & 0 & 9,15 \\
1 & 5 & 0 & 9,2 \\
1 & 6 & 1 & 10,5 \\
1 & 7 & 0 & 11 \\
2 & 1 & 0 & 4,1 \\
2 & 2 & 0 & 6 \\
2 & 3 & 0 & 7 \\
2 & 4 & 1 & 8,3 \\
2 & 5 & 0 & 9,2 \\
2 & 6 & 0 & 10 \\
2 & 7 & 1 & 9,9 \\
$\ldots$ & $\ldots$ & $\ldots$ & $\ldots$ \\
\hline
\end{tabular}

Figura 5.2: Exemplo da estruturação do banco de dados de desnutrição.

A resposta binária é representada por $y_{i j}$, com $y_{i j}=1$ se a $i$-ésima criança no período $j$ foi classificada com desnutrição grave; e zero, caso contrário. Considerando a resposta $y_{i j}$, o modelo tradicional é dado por

$$
\operatorname{logit}\left[P\left(y_{i j}=1\right)\right]=\beta_{0}+\beta_{1} z_{i j}
$$

e o modelo logístico misto por

$$
\operatorname{logit}\left[P\left(y_{i j}=1 \mid\left(\alpha_{1 i}, \alpha_{2 i}\right)\right)\right]=\beta_{0}+\beta_{1} z_{i j}+\alpha_{1 i}+\alpha_{2 i} z_{i j}
$$

sendo os parâmetros desconhecidos dos modelos (5.1) e (5.2): $\beta_{0}$ o intercepto fixo e $\beta_{1}$ a inclinação fixa associada a $z_{i j}$. Para o modelo misto, $\alpha_{1 i}$ é o intercepto aleatório e $\alpha_{2 i}$ é a inclinação aleatória do $i$-ésimo grupo, com $i=1, \ldots, 120$, que também são parâmetros desconhecidos. Em função do
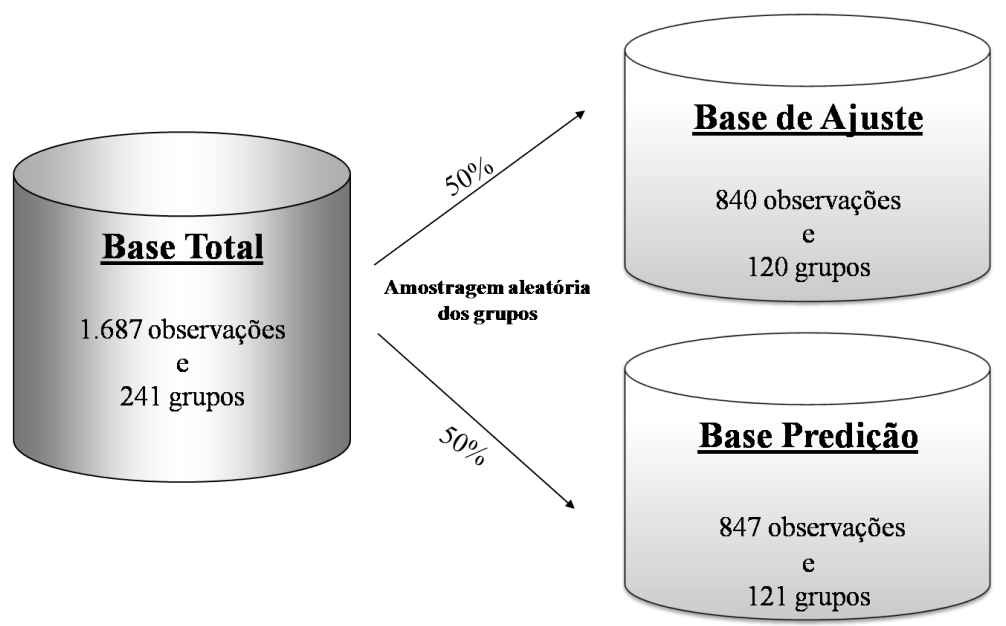

Figura 5.3: Amostra de ajuste e predição dos dados de desnutrição. 
peso da criança, a covariável $z_{i j}$ presente nos modelos (5.1) e (5.2) foi calculada da seguinte forma:

$$
z_{i j}=\operatorname{peso}_{i j}-\left(\bar{w}_{. j}-\sigma_{. j}\right)
$$

em que $\bar{w}_{. j}=\sum_{i=1}^{q} \operatorname{peso}_{i j} / q$ denota o peso médio para o $j$-ésimo período,

$$
\sigma_{. j}=\sqrt{\sum_{i=1}^{q}\left(\operatorname{peso}_{i j}-\bar{w}_{. j}\right)^{2} / q}
$$

denota o desvio-padrão do peso para o $j$-ésimo período com $i$ indexando a criança. Esta variável pode ser interpretada como a distância do peso da criança em relação a média do $j$-ésimo período menos 1 desvio-padrão do $j$-ésimo período. Desta forma, crianças com valor $z_{i j}$ negativo estariam muito abaixo do peso esperado para o $j$-ésimo período, sendo um forte indicativo de desnutrição.

A seguir, apresentam-se as estimativas dos parâmetros provenientes do modelo tradicional e misto, obtidas a partir da BA. Posteriormente, avaliou-se a predição da resposta na BA, utilizando a função logito para ambos os modelos. Na BP, aplicou-se os métodos de predição e avaliou-se a predição da resposta do modelo tradicional e os métodos de predição do modelo misto.

\subsubsection{Estimativas dos Parâmetros}

A Tabela 5.1 apresenta as estimativas dos parâmetros dos modelos (5.1), ajustado pelo método da máxima verossimilhança usando o algoritmo de Escore de Fisher, e (5.2), ajustado pelo método de estimação AL. Analisando os efeitos fixos dos modelos, o intercepto $\left(\beta_{0}\right)$ e o coeficiente de regressão $\left(\beta_{1}\right)$ foram significativos ( $\mathrm{p}$-valor $\left.<0,05\right)$. O coeficiente fixo $\beta_{1}$ associado a $z_{i j}$ apresentou sinal negativo em ambos os modelos, indicando que quando há incremento de uma unidade em $z_{i j}$, menor é a probabilidade da criança apresentar desnutrição grave.

Tabela 5.1: Estimativas dos parâmetros do modelo tradicional e misto para os dados de desnutrição.

\begin{tabular}{llcc}
\hline Modelo tradicional & Estimativa & Erro padrão & P-valor \\
\hline$\beta_{0}$ & 1,132 & 0,150 & $<0,001$ \\
$\beta_{1}$ & $-2,543$ & 0,186 & $<0,001$ \\
\hline \hline Modelo misto & Estimativa & Erro padrão & P-valor \\
\hline$\beta_{0}$ & 1,635 & 0,390 & $<0,001$ \\
$\beta_{1}$ & $-5,294$ & 0,594 & $<0,001$ \\
Desvio-padrão dos efeitos aleatórios $\left(\sigma_{1}, \sigma_{2}\right)$ & $(2,226,3,212)$ & & \\
Correlação entre os efeitos aleatórios $\alpha_{1}$ e $\alpha_{2}$ & $-0,167$ & & \\
\hline
\end{tabular}

Na mesma tabela para o modelo misto, pode-se observar que o desvio-padrão dos valores estimados para o intercepto aleatório foi menor do que valores estimados para o coeficiente de regressão aleatório, i.e, $\sigma_{1}<\sigma_{2}$, indicando que a inclinação aleatória apresentou maior dispersão nos seus valores do que o intercepto aleatório. Ao analisar a correlação entre os efeitos aleatórios $\alpha_{1}$ e $\alpha_{2}$, 
pode-se verificar que o intercepto aleatório e o coeficiente de regressão aleatório apresentaram uma baixa correlação negativa.

Na Figura 5.4, são fornecidos os gráficos dos valores estimados do intercepto aleatório por criança (grafico (a)), dos valores estimados da inclinação aleatória por criança (grafico (b)), de dispersão dos efeitos aleatórios (grafico (c)), qq-plot normal do intercepto aleatório (grafico (d)) e qq-plot normal da inclinação aleatória (grafico (e)), segundo o modelo (5.2) na BA. Nota-se nos gráficos (d) e (e) para cada efeito aleatório que, descritivamente, há fuga da suposição de normalidade. Porém, assumindo que o modelo (5.2) é o mais adequado aos dados, serão ajustados os métodos de predição com o objetivo de analisar a eficácia dos métodos de predição em relação à suposição de normalidade.
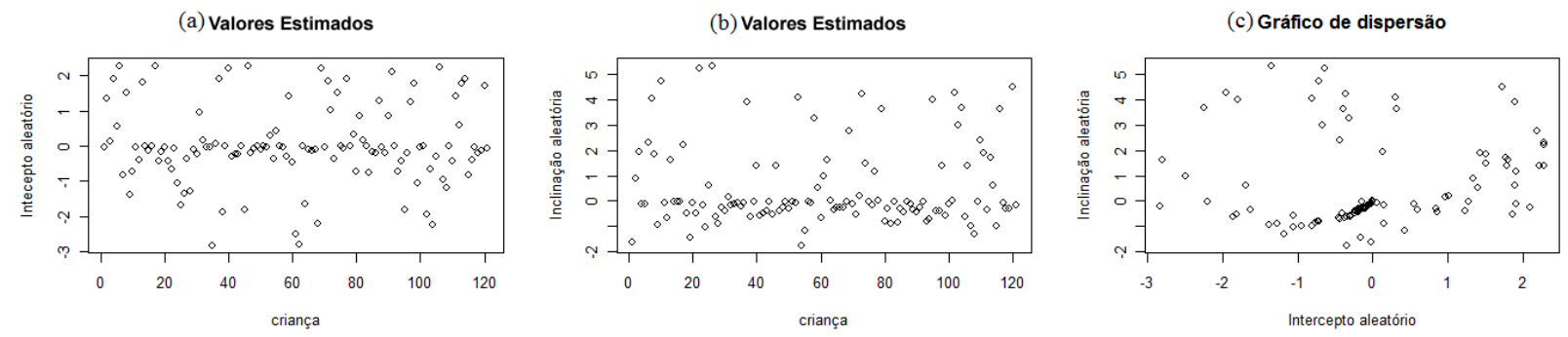

(d) QQ-plot Normal: Intercepto aleatório

(e) QQ-plot Normal: Inclinaçäo aleatória
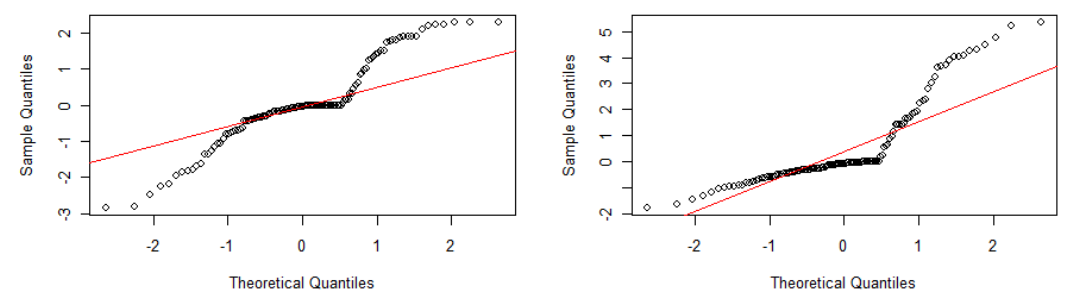

Figura 5.4: Método de Estimação Laplace dos dados de desnutrição: (a) valores estimados do intercepto aleatório por criança; (b) valores estimados da inclinação aleatória por criança; (c) gráfico de dispersão dos efeitos aleatórios; (d) qq-plot normal do intercepto aleatório; (e) qq-plot normal da inclinação aleatória, na BA.

\subsubsection{Desempenho preditivo}

O desempenho preditivo dos modelos, na base de ajuste e predição, é apresentado nesta subseção baseado nas medidas AUC e KS. Como a predição da resposta do modelo é dada por uma probabilidade, a classificação em resposta binária dependeu de um determinado ponto de corte. Para o cálculo da medida KS, classificou-se a probabilidade predita em resposta binária pelo ponto de corte ótimo de cada modelo, que minimizou seus respectivos erros de classificação.

$\mathrm{Na} \mathrm{BA}$, como os efeitos aleatórios são conhecidos, obteve-se excelente nível de predição com $\mathrm{AUC}=98,5 \%$ e $\mathrm{KS}=87,7 \%$ para o modelo logístico misto. No caso do modelo tradicional, os valores foram $\mathrm{AUC}=90,1 \%$ e $\mathrm{KS}=67,3 \%$, que ainda representam um nível ótimo de predição. 
Para a predição utilizando o método MPE dado por (3.48), considerou-se $y_{i}$. conforme (3.49). Neste caso, os dados foram agrupados no nível de grupo (criança), e a covariável no nível do grupo foi obtida pela média do peso dos sete períodos observados de cada criança. No caso do método de predição via modelos de regressão, RL e NP, foi utilizada a mesma covariável usada na obtenção de $y_{i}$. No caso do RL a covariável no nível de grupo entrou no modelo em sua forma original e no modelo não-paramétrico utilizou-se a função de suavização loess aplicada à covariável, conforme descrita na seção 3.3.3.2 no Capítulo 3. Os modelos RL e NP foram ajustados na BA, e as respectivas equações dos modelos foram aplicadas na BP, conforme passo (c) - (e) da Figura 3.1.

Considerando agora o conjunto de novas crianças (base de predição), foram aplicados os métodos de predição, e a Tabela 5.2 apresenta as medidas de desempenho AUC e KS para cada um destes métodos. O método NP apresentou os melhores resultados em termos de predição, enquanto o método MZ e tradicional apresentaram os piores resultados. Os métodos MPE e RL apresentaram um desempenho intermediário, entre os métodos NP e MZ.

Em particular, quando se analisou o KS, o método MPE apresentou valor um pouco superior ao RL, e quando se considerou o AUC ocorre o inverso. Entretanto, todos os métodos de predição apresentaram o mesmo patamar de discriminação, por exemplo, os valores de KS entre 52,1\% e $53,4 \%$, o qual representa um nível muito bom de discriminação.

Tabela 5.2: Medidas de desempenho dos métodos de predição (tradicional, MPE, RL, NP e MZ), na BP para os dados de desnutrição.

\begin{tabular}{lcc}
\hline Método de predição & AUC & KS \\
\hline Tradicional & $84,0 \%$ & $52,1 \%$ \\
MPE & $84,1 \%$ & $53,1 \%$ \\
RL & $84,3 \%$ & $52,6 \%$ \\
NP & $84,4 \%$ & $53,4 \%$ \\
MZ & $84,0 \%$ & $52,1 \%$ \\
\hline
\end{tabular}

As conclusões que podem ser obtidas a partir dos resultados apresentados na Tabela 5.2 são similares às obtidas no cenário 5 dos estudos de simulação (Capítulo 4), no qual observou-se no cenário 5 que os métodos de predição apresentam mesmo nível de discriminação quando: a correlação entre os efeitos aleatórios foi baixa, o tamanho do grupo foi pequeno em relação ao tamanho da base total e a parte fixa possuía um "peso"similar à parte aleatória.

No cenário 5, o método NP foi aquele que apresentou o pior desempenho em relação aos demais métodos. Nesta aplicação, conforme Figura 5.4, gráficos (d) e (e), os efeitos aleatórios empíricos não apresentaram normalidade, e devido a este fato, possivelmente o método NP apresentou melhor resultado em relação aos demais métodos. Mesmo assim, os métodos MPE e RL mostraramse eficientes mesmo quando a suposição da normalidade não está satisfeita. Nota-se ainda que as metodologias que anularam a existência da parte aleatória do modelo, tradicional e MZ, foram aquelas que apresentaram os piores níveis preditivos, indicando que as presenças dos efeitos aleatórios auxiliam na discriminação da resposta. 


\subsection{Aplicação em Marketing: Modelo de venda de um produto adicional}

Nesta seção, apresenta-se uma aplicação na área de marketing, usando um banco de dados de uma empresa de telecomunicações.

Em marketing, o modelo de cross-sell ou modelo de venda cruzada é utilizado para oferta de itens que complementam a lista de produtos ou serviços de um determinado cliente. O objetivo de fazer a "venda cruzada" seria aumentar a rentabilidade do cliente ou melhorar a relação dele com a empresa, fazendo com que o mesmo tenha um vínculo maior com a companhia. O cross-selling é um dos temas mais importantes dentro das estratégias de CRM, e sua importância no planejamento das campanhas de marketing tem sido discutida por diversos autores, como por exemplo, Butera (2000) e Kamakura et al. (2003).

Esta aplicação, trata de uma empresa de telefonia móvel que deseja fazer a abordagem de seus clientes para venda de um serviço adicional via canal de telemarketing. Este canal de vendas tem um custo muito elevado e uma capacidade limitada de chamadas a serem realizadas durante um determinado período. Assim, um modelo estatístico poderia ajudar a selecionar uma quantidade mínima de pessoas com máxima eficácia nas vendas. A partir deste modelo, seria possível atribuir uma probabilidade de compra do serviço adicional a cada celular ou terminal telefônico, e com base nesta probabilidade, cada terminal poderia ser ordenado e priorizado na abordagem de venda do serviço.

A probabilidade de compra, obtida a partir de um modelo de regressão logística, tem sido usada quando as unidades amostrais são independentes. Para um banco de dados com estrutura hierárquica, nos quais as observações não são mais independentes, seria apropriado considerar um modelo logístico misto para gerar esta probabilidade. Nesta aplicação, o banco de dados apresenta estrutura hierárquica, em que clientes corporativos possuem empregados que usam o celular para fins comerciais, conforme ilustração na Figura 5.5.

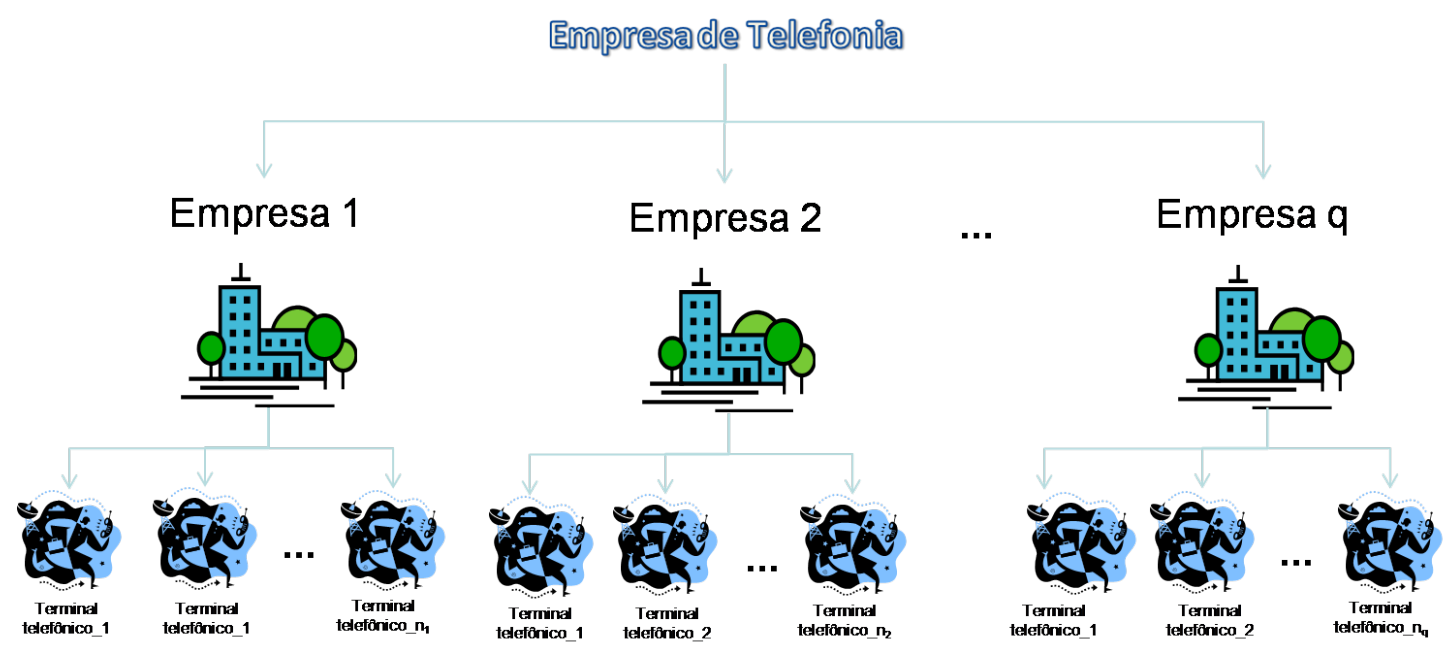

Figura 5.5: Estrutura hierárquica do banco de dados de telefonia.

O produto adicional considerado nesta aplicação é o SMS (short message service). Este é um 
serviço de mensagens que permite que o aparelho celular troque mensagens curtas de texto com outros aparelhos. A empresa de telefonia oferece três formas de se contratar o serviço: pagamento individual por mensagem, pagamento diário e assinatura mensal. Se o serviço é contratado por dia ou por mês, as pessoas podem enviar SMS ilimitados dentro do período contratado.

O pacote mensal é o mais econômico dentre as três formas de utilização do serviço, uma vez que o proprietário utilize o serviço com frequência. A campanha de marketing será focada na venda do pacote mensal de SMS para as linhas que ainda não possuem esse serviço.

Como a empresa de telecomunicações armazena o conjunto de dados mensalmente, a análise de dados foi realizada com base em um mês específico, denominado mês de referência. O comportamento dos clientes corporativos e/ou respectivos celulares foi representado por informações transacionais (histórico de utilização do celular) e cadastrais. Estas informações foram consideradas como covariáveis a serem testadas do modelo e foram observadas do mês de referência para trás, por um período de até 12 meses de histórico. O objetivo do problema é prever o valor da variável resposta um mês depois do mês de referência com base nas covariáveis. A resposta binária foi definida como:

- 1 - linha adquiriu o pacote SMS mensal um mês depois do mês de referência;

- 0 - linha não adquiriu o pacote de SMS mensal um mês depois do mês de referência.

As informações usadas como covariáveis foram fornecidas tanto no nível de cliente corporativo (empresa) como no nível de celular (terminal telefônico). Exemplos de covariáveis por nível hierárquico são listados a seguir:

- Empresa: localização geográfica, tempo de fundação da empresa, ramo de atividade, tempo da empresa na operadora de telefonia móvel, quantidade de terminais e valor da fatura.

- Celular: tempo do terminal, indicador do uso de pacote de dados, indicador de utilização de e-mail, indicador do uso de SMS individual, indicador do uso de SMS diário, indicador de posse de SMS mensal no passado, minutos de chamadas recebidas, minutos de chamadas realizadas, minutos roaming, percentual de minutos que o terminal representa dentro da empresa e percentual em reais $(\mathrm{R} \$)$ que o terminal representa dentro da fatura da empresa.

Foram considerados dois períodos de análise, junho/2010 e dezembro/2010. Os dados com referência em junho/2010 foram definidos como BA, no qual obteve-se as estimativas dos parâmetros. Os dados com referência em dezembro/2010 foram considerados para analisar a predição da resposta em um período futuro, com base no modelo ajustado em junho/2010, e esta base foi considerada como BP.

Na Figura 5.6 observa-se a quantidade de clientes corporativos e celulares (ou terminais telefônicos) em cada período. Em junho/2010, havia 19.473 terminais aninhados em 485 empresas e, em dezembro/2010, 21.050 terminais aninhados em 657 empresas. Na base de dados de dezembro/2010, 29\% eram novos clientes e os demais já existiam na base de junho/2010. 


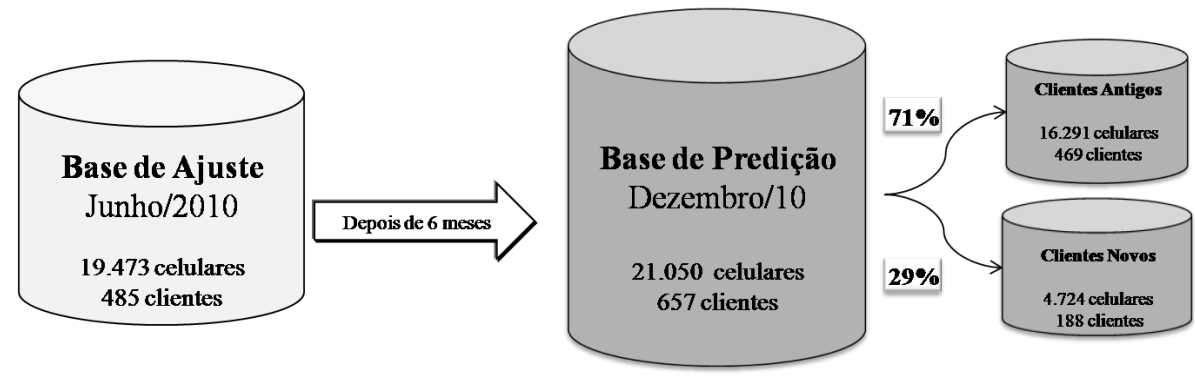

Figura 5.6: Base de ajuste (junho/2010) e predição (dezembro/2010) dos dados de telefonia.

Observa-se que o problema de predição desta aplicação difere da aplicação da seção anterior, pois o mesmo cliente corporativo (grupo) pode aparecer na base de ajuste e predição. Assim, a predição em um período futuro foi dividida em duas partes, para novos grupos e para grupos já existentes. A predição futura para empresas já existentes na BA foi realizada da maneira direita, pela função logito, pois eram conhecidos os valores individuais dos efeitos aleatórios. No caso das novas empresas, a predição da resposta foi realizada utilizando os métodos de predição propostos neste trabalho.

Diferente do estudo apresentado na seção anterior, este banco de dados possui quantidade desbalanceada de unidades amostrais por grupo. Na Tabela 5.3 pode-se observar a distribuição de celulares dentro das empresas, para os períodos de junho/2010 e dezembro/2010. Nota-se que metade dos clientes possui aproximadamente até 25 aparelhos celulares, e nos casos mais extremos, há empresas que tem mais de 440 terminais.

Tabela 5.3: Medidas Resumo para os dados de telefonia: Quantidade de terminais por cliente corporativo.

\begin{tabular}{rcccccc}
\hline Base de Dados & Mínimo & $\mathbf{1}^{\mathbf{0}}$ Quartil & Mediana & Média & $\mathbf{3}^{\mathbf{o}}$ Quartil & Máximo \\
\hline Junho/2010 & 1,00 & 22,00 & 28,00 & 40,15 & 42,00 & 487,00 \\
Dezembro/2010 & 1,00 & 19,00 & 23,00 & 32,04 & 35,00 & 442,00 \\
\hline
\end{tabular}

Nas análises realizadas no mercado não é comum utilizar o modelo logístico misto para fazer a predição da resposta em período futuro, e o modelo tradicional, que ignora a existência dos efeitos aleatórios, tem sido usado de maneira paliativa mesmo quando o banco de dados apresenta estrutura hierárquica. Desta forma, além de comparar os métodos de predição do modelo logístico misto entre si, como análise complementar, considerou-se o também o modelo tradicional.

A resposta binária do modelo é dada por $y_{i j}$, com $y_{i j}=1$ se a $j$-ésima linha pertencente à $i$-ésima empresa adquiriu o pacote de SMS; e zero, caso contrário. Considerando a resposta $y_{i j}$, o modelo tradicional é dado por

$$
\operatorname{logit}\left[P\left(y_{i j}=1\right)\right]=\beta_{0}+\mathbf{x}_{i j}^{t} \boldsymbol{\beta}
$$

e o modelo misto por

$$
\operatorname{logit}\left[P\left(y_{i j}=1\right) \mid \boldsymbol{\alpha}_{i}\right]=\beta_{0}+\mathbf{x}_{i j}^{t} \boldsymbol{\beta}+\alpha_{1 i}+z_{i j} \alpha_{2 i},
$$


em que os parâmetros desconhecidos dos modelos (5.3) e (5.4) são: $\beta_{0}$, o intercepto fixo; $\boldsymbol{\beta}$, o vetor de efeitos fixos associado ao vetor de covariáveis conhecidas $\mathbf{x}_{i j}$, descritas no início desta subseção no nível de empresa e terminal telefônico. Para o modelo misto, $\alpha_{1 i}$ é o intercepto aleatório e $\alpha_{2 i}$ é a inclinação aleatória da $i$-ésima empresa, com $i=1, \ldots, 485$, que são parâmetros desconhecidos. A covariável $z_{i j}$ associada à inclinação aleatória no modelo misto é o percentual de minutos que o terminal $j$ representa dentro da empresa $i$.

A seguir, apresentam-se as estimativas dos parâmetros provenientes do modelo tradicional e misto obtidas pela BA. Posteriormente, avaliou-se a predição da resposta nas BA e BP.

\subsubsection{Estimativas dos Parâmetros}

Supõe-se que o modelo ajustado está corretamente especificado no sentido de selecionar o melhor conjunto de variáveis explicativas, tanto parte fixa quanto na parte aleatória do modelo misto.

As estimativas dos parâmetros do modelo (5.3) foram obtidas pelo método da máxima verossimilhança usando o algoritmo de Escore de Fisher, e as estimativas do modelo (5.4) pelo método de estimação AL.

Como cada categoria das variáveis explicativas entram no modelo como variáveis indicadoras (dummies), foi realizada uma Análise de variância (ANOVA), na qual a hipótese nula do teste foi de modelos aninhados para verificar a significância de cada fator (ou de cada variável explicativa) na presença de outros fatores. Na Tabela 5.4, os p-valores dos fatores do modelo tradicional e misto foram obtidos através da estatística de Qui-quadrado. Analisando a tabela, todos os fatores foram significantes ( $\mathrm{p}$-valor $<0,05$ ) para ambos os modelos.

Tabela 5.4: Anova do modelo tradicional e misto para os dados de telefonia: Análise do p-valor.

\begin{tabular}{lrr}
\hline Variável & Modelo tradicional & Modelo misto \\
\hline Quantidade de dias de utilização de SMS & $<0,001$ & $<0,001$ \\
Indicador de posse SMS mensal no passado & $<0,001$ & $<0,001$ \\
Minutos roaming & $<0,001$ & 0,046 \\
Indicador de uso de e-mail & $<0,001$ & 0,010 \\
Indicador de uso de pacote de dados & 0,010 & $<0,001$ \\
\hline
\end{tabular}

Para cada variável explicativa, a primeira categoria foi considerada como célula de referência. No caso das variáveis SMS e roaming, as categorias de referência são "quantidade de dias de utilização de SMS <2" e "minutos roaming =0", respectivamente. As demais variáveis entraram no modelo como variáveis indicadoras. Os parâmetros referentes às inclinações fixas do modelo estão associados às categorias de cada variável indicadora da seguinte forma:

- $\beta_{1}$ : quantidade de dias de utilização de $\mathrm{SMS}=2$,

- $\beta_{2}$ : quantidade de dias de utilização de $\operatorname{SMS}>2$, 
- $\beta_{3}$ : indicador de posse SMS mensal no passado,

- $\beta_{4}$ : minutos roaming $\left.] 0,10\right]$,

- $\beta_{5}$ : minutos roaming $>10$,

- $\beta_{6}$ : indicador de uso de $e$-mail e

- $\beta_{7}$ : indicador de uso de pacote de dados.

As estimativas dos parâmetros, erros padrão (e.p.) e p-valores do modelo tradicional e misto ajustados podem ser observados na Tabela 5.5. Observa-se que todos efeitos fixos foram significativos, com exceção de $\beta_{5}$. Entretanto, pela Tabela 5.4, o fator "minutos roaming" foi significante nos dois modelos, e por isso $\beta_{5}$ não foi retirado dos modelos.

Em geral, as variáveis presentes nos modelos, que estão relacionadas ao uso de outros serviços, explicam positivamente a compra do pacote de SMS. Por exemplo, como a estimativa de $\beta_{3}$ apresentou valor positivo, pode-se interpretar que se o funcionário já teve SMS no passado, a probabilidade dele adquirir novamente um pacote de SMS aumenta.

Tabela 5.5: Estimativas dos parâmetros, erro padrão e p-valor para modelo tradicional e misto para os dados de telefonia.

\begin{tabular}{lccc}
\hline Modelo tradicional & Estimativa & Erro Padrão & P-valor \\
\hline$\beta_{0}$ & $-4,898$ & 0,089 & $<0,001$ \\
$\beta_{1}$ & 1,258 & 0,166 & $<0,001$ \\
$\beta_{2}$ & 1,675 & 0,289 & $<0,001$ \\
$\beta_{3}$ & 2,34 & 0,307 & $<0,001$ \\
$\beta_{4}$ & 0,699 & 0,178 & $<0,001$ \\
$\beta_{5}$ & 0,300 & 0,261 & 0,250 \\
$\beta_{6}$ & 1,542 & 0,346 & $<0,001$ \\
$\beta_{7}$ & 0,788 & 0,288 & 0,006 \\
\hline \hline Modelo misto & Estimativa & Erro Padrão & P-valor \\
\hline$\beta_{0}$ & $-9,308$ & 0,468 & $<0,001$ \\
$\beta_{1}$ & 0,594 & 0,235 & 0,011 \\
$\beta_{2}$ & 1,074 & 0,378 & 0,004 \\
$\beta_{3}$ & 1,632 & 0,404 & $<0,001$ \\
$\beta_{4}$ & 0,564 & 0,247 & 0,022 \\
$\beta_{5}$ & $-0,040$ & 0,350 & 0,910 \\
$\beta_{6}$ & $-0,713$ & 0,437 & 0,100 \\
$\beta_{7}$ & 1,968 & 0,435 & $<0,001$ \\
\hline desvio-padrão dos ef. aleatórios $\left(\sigma_{1}, \sigma_{2}\right)$ & $(5,670,2,380)$ & & \\
Correlação entre os efeitos aleatórios $\alpha_{1}$ e $\alpha_{2}$ & $-0,680$ & & \\
\hline
\end{tabular}

A variável $e$-mail associada ao parâmetro $\beta_{6}$ está na fronteira do limite de nível de significância e não foi removida do modelo misto para que a parte fixa de ambos os modelos considerassem o mesmo conjunto de variáveis. É importante salientar que a covariável $z_{i j}$ foi testada no modelo tradicional, mas não foi significante.

Ainda na Tabela 5.5, observou-se que o valor do desvio-padrão do intercepto aleatório $\left(\sigma_{1}\right)$ é maior do que o valor do desvio-padrão da inclinação aleatória $\left(\sigma_{2}\right)$. Além disso, os valores dos 
desvios-padrão tanto do intercepto aleatório quanto da inclinação aleatória indicam alta variabilidade dos efeitos aleatórios. A correlação de -0,68 resulta em uma média-alta correlação negativa.

\subsubsection{Desempenho preditivo}

A predição da resposta para a base de ajuste (junho/2010) apresentou valor de predição em níveis excelentes, com $\mathrm{KS}=87,3 \%$ e $\mathrm{AUC}=97,7 \%$, enquanto o modelo tradicional apresentou valores de $\mathrm{KS}=39,0 \%$ e $\mathrm{AUC}=71,3 \%$, que ainda representam um nível de discriminação consideravelmente bom. Comparando o poder preditivo dos dois modelos, verificou-se a presença dos efeitos aleatórios, quando de fato existe uma estrutura hierárquica nos dados, favorece a predição da resposta.

Para a predição na base de dezembro/2010 (base de predição), utilizando o método MPE, dado por (3.48), considerou-se $y_{i .}=n_{i .} / 2$, conforme abordagem de Tamura e Giampaoli (2010). No caso do método de predição via modelos de regressão, RL e NP, os dados foram agrupados no nível de grupo (empresa). Todas as covariáveis agregadas no nível de grupo foram testadas nos modelos, e depois de rodar o método stepwise, selecionou-se um conjunto de covariáveis que melhor explicaram aos efeitos aleatórios.

Nas Tabelas 5.6 e 5.7 são listadas as covariáveis presentes nos modelos para o intercepto aleatório e para a inclinação aleatória, segundo os modelos RL e NP, respectivamente. Para cada tabela, as covariáveis associadas a palavra 'sim' indicam que a variável entrou no modelo em sua forma original e aquela marcadas com 's(sim)' indicam que entraram no modelo usando uma função de suavização smoothing splines, descrita na seção 3.3.3.2, no Capítulo 3.

Tabela 5.6: Variáveis presentes nos modelos RL e NP para variável resposta intercepto aleatório, para os dados de telefonia.

\begin{tabular}{lcc}
\hline Variáveis do modelo para o Intercepto Aleatório & RL & NP \\
\hline Fatura média dos equipamentos & & $\mathrm{s}(\operatorname{sim})$ \\
Média de minutos recebidos & $\mathrm{sim}$ & $\mathrm{sim}$ \\
Posse de SMS avulso & $\mathrm{sim}(\operatorname{sim})$ & $\mathrm{s}(\operatorname{sim})$ \\
Posse de SMS diário & $\mathrm{sim}$ & $\mathrm{s}(\operatorname{sim})$ \\
Posse de SMS histórico & $\mathrm{sim}$ & \\
Quantidade de contratos com SMS mensal & $\mathrm{sim}$ & $\mathrm{s}(\operatorname{sim})$ \\
Quantidade de linhas do cliente & $\mathrm{sim}$ & $\mathrm{sim}$ \\
Receita total dos equipamentos & $\mathrm{sim}$ & $\operatorname{sim}$ \\
Soma de minutos recebidos & & $\operatorname{sim}$ \\
Soma de minutos totais &
\end{tabular}

Os modelos RL e NP foram ajustados na BA, e as respectivas equações dos modelos foram aplicadas na BP, conforme passo (c) - (e) da Figura 3.1. Nota-se que os modelos para o intercepto aleatório exigiram mais variáveis independentes do que os modelos para a inclinação aleatória. Além disso, para ambos os casos, as covariáveis que tinham maior relação com o evento resposta, geralmente estavam relacionadas às informações históricas de SMS. Observa-se também que informações adicionais que não apareceram no modelo logístico misto, entraram nos modelos ajustados para os efeitos aleatórios como, por exemplo, fatura média dos equipamentos, quantidade linhas dentro 
Tabela 5.7: Variáveis presentes nos modelos RL e NP para variável resposta inclinação aleatória, para os dados de telefonia.

\begin{tabular}{lcc}
\hline Variáveis do modelo para a Inclinação Aleatória & RL & NP \\
\hline Média de minutos recebidos & & sim \\
Média de minutos roaming & & $\mathrm{s}(\operatorname{sim})$ \\
Posse de SMS diário & sim & \\
Posse de SMS histórico & sim & \\
Quantidade de contratos com SMS mensal & sim & $\mathrm{s}(\operatorname{sim})$ \\
Quantidade linhas dentro cliente & & sim \\
Soma de minutos recebidos & & \\
\hline
\end{tabular}

cliente, receita total dos equipamentos, minutos recebidos, minutos totais, etc.

Na base de predição (dezembro/2010), como o valor dos efeitos aleatórios são conhecidos para clientes antigos (obtidos no ajuste do modelo, base de junho/2010), foi possível realizar a predição da resposta usando a função logito. Nota-se que mesmo se novas observações aparecessem na BP, desde que elas estejam associadas a um cliente antigo, conhece-se o valor de seus efeitos aleatórios.

$\mathrm{Na}$ BP para clientes antigos, as medidas de desempenho AUC e KS para o modelo misto apresentaram valores iguais a $58,1 \%$ e $81,1 \%$, respectivamente. No caso do modelo tradicional, obtiveram-se os valores $\mathrm{KS}=46,5 \%$ e $\mathrm{AUC}=73,7 \%$. Apesar de ambos os modelos apresentarem bom nível de desempenho, no caso do modelo misto o fato de se conhecer os valores individuais dos efeitos aleatórios, mesmo quando estimados em um período anterior, auxiliaram na qualidade da predição da resposta.

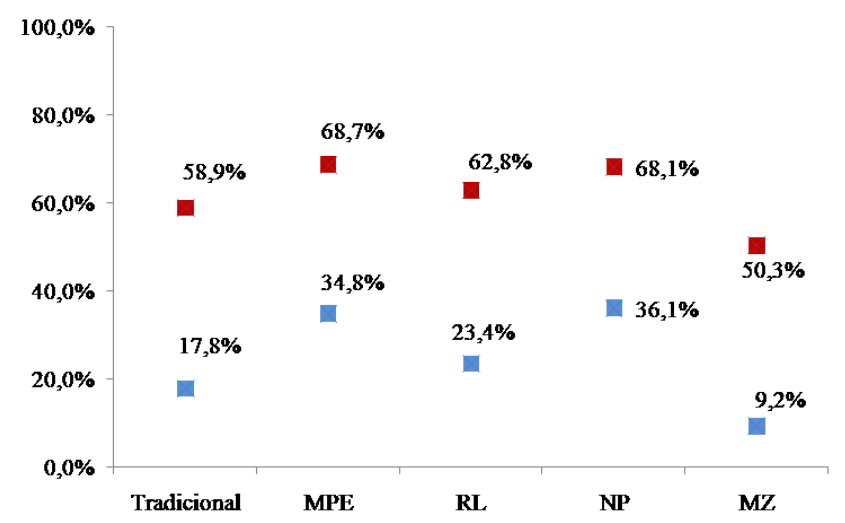

Figura 5.7: Medidas de desempenho AUC e KS na base de predição para novos grupos, por método de predição para os dados de telefonia.

O gráfico representado pela Figura 5.7, apresenta as medidas de desempenho para os clientes novos na base de predição (dezembro/2010), por método de predição, provenientes do modelo tradicional e misto. Para o modelo misto, foram utilizados os métodos de predição MPE, RL, NP e MZ, apresentados na Seção 3.

Pela análise deste gráfico, observou-se que os métodos de predição MPE e NP apresentaram desempenhos superiores aos demais, com valores de AUC (KS) acima de 68\% (34\%). No caso 
do método de predição $\mathrm{RL}, \mathrm{AUC}=62,8 \%$ e $\mathrm{KS}=23,4 \%$, que indicou um desempenho preditivo superior ao método $\mathrm{MZ}(\mathrm{AUC}=50,3 \%$ e $\mathrm{KS}=9,2 \%$ ). Por fim, os métodos de predição MPE, RL e NP apresentaram desempenhos superiores ao modelo tradicional, que por sua vez foi superior ao método MZ. Nota-se que a predição do modelo tradicional e método MZ apresentaram um baixo nível de discriminação, valores de KS $<20 \%$.

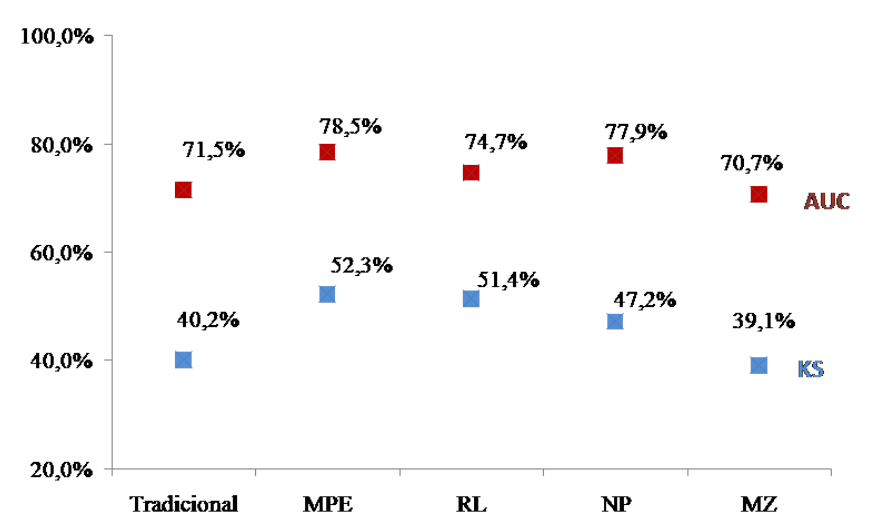

Figura 5.8: Medidas de desempenho AUC e KS na base de predição (todos: novos e antigos), por método de predição para os dados de telefonia.

O gráfico representado pela Figura 5.8 apresenta as medidas de desempenho para cada método de predição, para clientes antigos e novos (base total de dezembro/2010). Os índices de desempenho KS e AUC foram recalculados na base total com base nas mesmas probabilidades obtidas na predição de cada base individualmente (base de clientes antigos e base de clientes novos).

Observou-se neste gráfico, que os métodos de predição MPE, RL e NP apresentaram desempenhos superiores ao método $\mathrm{MZ}$ ( $\mathrm{AUC}=70,7 \%$ e $\mathrm{KS}=39,1 \%$ ), com valores de AUC (KS) superiores a $74 \%$ (47\%). Apesar do método RL ter resultado em um poder preditivo inferior ao MPE e NP na base de clientes novos, quando se considerou a base total, este método apresentou desempenho semelhante ao MPE e NP. Este fato pode ser explicado devido as probabilidades serem reordenadas na base total. Comparando o modelo tradicional com misto em termos de predição, observou-se, no geral, que os métodos de predição para o modelo misto apresentaram melhor desempenho preditivo, e o método MZ apresentou discriminação mais próxima e um pouco inferior ao modelo tradicional.

Na Figura 5.9, podem ser observados os gráficos: valores estimados do intercepto aleatório por cliente (gráfico (a)), valores estimados da inclinação aleatória por cliente (gráfico (b)), dispersão dos efeitos aleatórios (gráfico (c)), qq-plot normal do intercepto aleatório (gráfico (d)) e qq-plot normal da inclinação aleatória (gráfico (e)), na BA. Nota-se pelos gráficos (d) e (e) que os efeitos aleatórios empíricos não se aproximam da distribuição normal, fato que poderia ser justificado por parte dos dados possuírem pouca dispersão e outra parte grande dispersão. Apesar dos efeitos aleatórios não apresentarem normalidade em sua distribuição empírica, a predição das probabilidades futuras mostraram que os métodos de predição MPE e RL foram eficientes mesmo quando os efeitos aleatórios empíricos não apresentaram normalidade. O método NP foi aquele que apresentou 
melhor desempenho dentre todos os métodos de predição.

(a) Valores Estimados

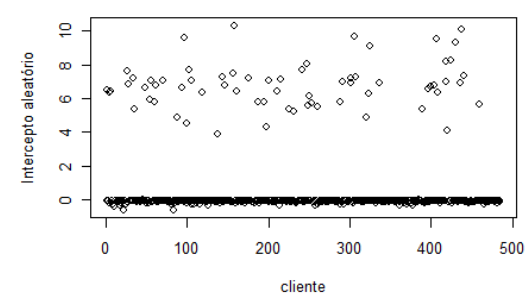

(d) QQ-plot Normal: Intercepto aleatório

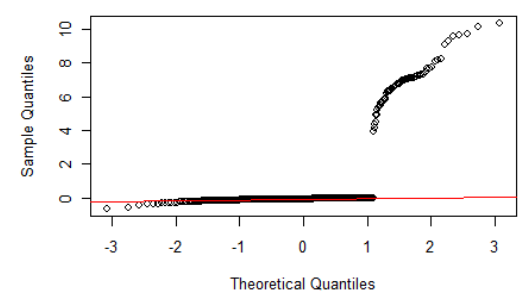

(b) Valores Estimados

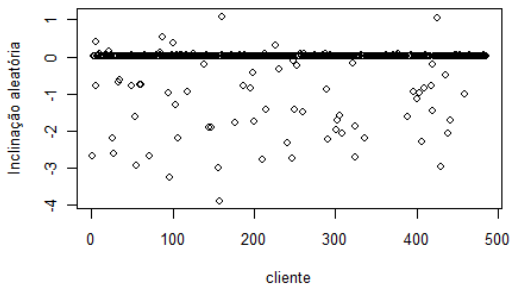

(e) QQ-plot Normal: Inclinação aleatória

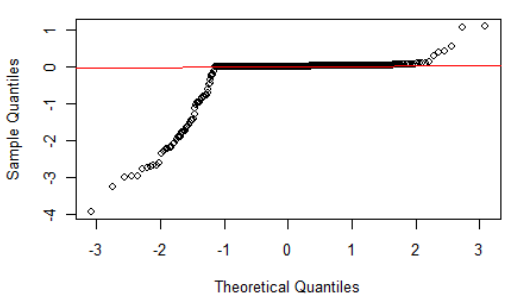

(c) Gráfico de Dispersão

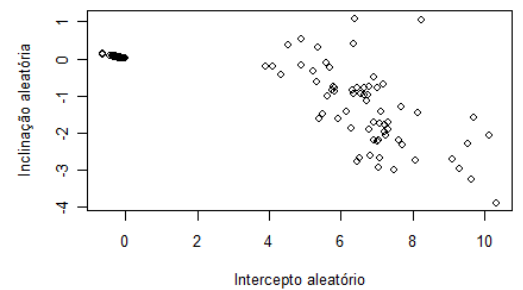

Figura 5.9: Método de Estimação Laplace dos dados de telefonia: (a) valores estimados do intercepto aleatório por cliente; (b) valores estimados da inclinação aleatória por cliente; (c) gráfico de dispersão dos efeitos aleatórios; (d) qq-plot normal do intercepto aleatório; (e) qq-plot normal da inclinação aleatória, na BA.

\section{Comentários Finais dos Estudos de Aplicação}

Com base nos resultados apresentados nos estudos de aplicação em banco de dados com estrutura hierárquica, as propostas de predição desenvolvidas para o modelo logístico misto foram úteis na solução de problemas distintos que envolveram a predição da resposta para novos grupos.

Em ambas as aplicações, os efeitos aleatórios empíricos apresentaram afastamento em relação à normalidade, e devido a este fato, possivelmente o método NP apresentou melhor resultado em termos preditivos comparado com os outros métodos. Mesmo assim, os métodos MPE e RL mostraram-se eficientes na predição da resposta. Por fim, as metodologias que anularam a existência da parte aleatória do modelo, tradicional e MZ, foram aquelas que apresentaram os piores níveis preditivos, indicando que as presenças dos efeitos aleatórios auxiliaram na predição da resposta. 


\section{Capítulo 6}

\section{Conclusões}

\subsection{Considerações Finais}

O problema de predição, quando o modelo logístico misto considera apenas o intercepto aleatório, já foi tratado na literatura pelos autores Jiang e Lahiri (2001), Skrondal e Rabe-Hesketh (2009) e Tamura e Giampaoli (2010).

O objetivo do presente trabalho foi propor métodos de predição para o modelo logístico misto com $k$ efeitos aleatórios, inserindo também as inclinações aleatórias no contexto de predição de observações futuras. Assim, quando existe o interesse em considerar variáveis explicativas na parte aleatória do modelo, inclinações aleatórias para cada grupo podem ser associadas a elas.

Para predição de novos grupos, apresentaram-se quatro metodologias, média zero (MZ), melhor preditor empírico (MPE), regressão linear (RL) e modelos não-paramétricos (NP), que foram avaliadas em estudos de simulação e em aplicações usando bancos de dados reais.

Nos estudos de simulação, a predição da resposta para novos grupos foi baseada na estimação dos parâmetros do modelo logístico misto pelos seguintes métodos de estimação: aproximação de Laplace (AL), quadratura adaptativa de Gauss-Hermite (QAGH) para dois e cinco pontos na quadratura, e quase-verossimilhança penalizada (QVP). Os métodos de estimação nos cenários considerados apresentaram alguns problemas como falta de convergência ou estimativas inconsistentes. Em particular, o método de QVP apresentou mais problemas na estimação dos parâmetros em relação aos demais métodos. O método AL, no geral, apresentou o menor viés nas estimativas dos parâmetros de interesse do modelo. Entretanto, foi constatado que apesar dos métodos de estimação apresentarem vieses consideráveis nas estimativas dos parâmetros, estes vieses não comprometeram a boa qualidade da predição da resposta para novos grupos.

Resultados dos estudos de simulação mostraram que o método MPE foi o mais eficaz em termos predição, porém apresentou alta complexidade computacional devido ao cálculo de integrais multidimensionais. Esse método, dependendo da estratégia utilizada para obter-se $y_{i}$, pode ou não depender de covariáveis. As vantagens dos métodos MZ, RL e NP em relação ao MPE foram que os métodos não apresentaram custo computacional no cálculo das probabilidades preditas, pois foram aplicadas de maneira analítica.

O método MZ, que considera a parte aleatória do modelo nula, mostrou-se em alguns casos tão eficiente quanto o MPE; assim como o método RL para os casos em que os efeitos aleatórios 
representaram uma "maior importância"no preditor linear do modelo. O pior desempenho foi observado para o método NP, porém a avaliação do método pode ter sido prejudicada devido aos estudos de simulação considerarem o modelo logístico misto com efeitos aleatórios seguindo apenas a distribuição normal.

Os métodos MZ, RL e NP podem ser usados para qualquer classe de modelos com efeitos aleatórios, independente de pertencer ou não à classe dos MLGM. Por outro lado, há algumas desvantagens no uso das técnicas RL e NP. Por exemplo, uma característica do(s) método(s) é (são) que ele(s) depende(m) da relação das covariáveis em relação à variável resposta (efeito aleatório). Também é necessário ajustar $k$ modelos de regressão, pois cada modelo prediz um efeito aleatório a partir de um conjunto específico de variáveis explicativas. No caso do método MZ uma grande desvantagem seria quando uma covariável muito importante está na parte aleatória do modelo, pois a mesma acaba sendo anulada do modelo.

Nos estudos de aplicação, foram apresentados dois estudos práticos usando banco de dados reais, e o método de estimação considerado em ambas as aplicações foi a AL.

No banco de dados de desnutrição, considerou-se a covariável relacionada ao peso da criança para explicar a desnutrição severa classificada com base na altura da criança. Essas informações foram coletadas em sete períodos distintos de observação. Nesta aplicação, a variável aleatória estava presente parte fixa e aleatória do modelo logístico misto, representado a mesma estrutura de modelo apresentada nos estudos de simulação do Capítulo 4. MPE e NP foram os métodos que apresentaram melhores resultados em termos de predição. Foi constatado nesta aplicação que apesar dos efeitos aleatórios empíricos não apresentarem distribuição normal, o método MPE e RL mostram-se eficientes na predição da resposta mesmo quando houve falha desta suposição, apresentando os mesmos níveis de predição do método NP, que não supõe normalidade na distribuição empírica dos efeitos aleatórios.

Na aplicação em marketing, o banco de dados de telefonia móvel considerou um conjunto de covariáveis na parte fixa e uma covariável distinta na parte aleatória do modelo logístico misto. A predição em um período futuro foi dividida em duas partes, para novos grupos e grupos já existentes no período de ajuste do modelo. Para os grupos já existentes, a predição no período futuro foi realizada da maneira usual, pela função logito, enquanto para os novos grupos utilizaramse os quatro métodos de predição. Para os novos grupos, os métodos MPE e NP foram aqueles que apresentaram melhores resultados em termos de predição. Nesta aplicação, apenas o método MPE mostrou-se mais robusto quanto a suposição de normalidade.

Em suma, com base nos estudos de simulação e aplicação, os resultados mostraram que os métodos de predição propostos para o modelo logístico misto são relevantes na predição da resposta, pois além de apresentarem níveis preditivos, no geral, superiores ao modelo logístico tradicional, mostraram-se também eficientes na classificação da resposta binária.

Dentre todos os métodos de predição, o MPE foi o método que mostrou-se mais estável e eficiente em termos de discriminação da resposta. Este resultado é coerente, uma vez que este é o método teórico baseado na esperança condicional do modelo logístico misto. Os demais métodos, 
apesar de apresentarem desempenhos preditivos semelhantes ao MPE, são métodos heurísticos para o problema de predição de observações futuras que foram baseados na avaliação dos resultados encontrados. Estes métodos de aproximação assumiram uma solução próxima da ideal, mas desconsideram algum tipo de informação fornecido pelo modelo misto. Por exemplo, no caso da MZ, o método baseou-se apenas nos efeitos fixos estimados pelo modelo misto; e no caso dos métodos RL e NP, ignorou-se a existência da correlação entre os efeitos aleatórios.

\subsection{Sugestões para Pesquisas Futuras}

As metodologias de predição apresentadas para o modelo misto podem ser facilmente implementadas para os modelos multiníveis com três ou mais níveis hierárquicos.

No caso da metodologia MPE, a mesma pode ser estendida para outras distribuições pertencentes à família exponencial como Poisson, Gama, Multinomial, entre outras, com a flexibilidade de se trabalhar com distintas funções de ligação sendo elas canônicas ou não.

No caso da metodologia via modelos de regressão, os métodos RL e NP podem ser estendidos à classe dos modelos com resposta multivariada, que consideram a existência de correlação entre os efeitos aleatórios.

McCulloch e Neuhaus (2011) investigaram o impacto na qualidade da predição sob a especificação incorreta da distribuição dos efeitos aleatórios que incluem também o problema de inferência sobre os parâmetros das covariáveis, a predição dos efeitos aleatórios e a estimação das componentes de variância dos efeitos aleatórios. No artigo Albert (2012) este problema foi aplicado a dados longitudinais com resposta binária. Neste caso, poderia ser estudado o impacto da especificação incorreta da distribuição dos efeitos aleatórios sobre a inferência dos parâmetros e a predição da resposta.

Finalmente, os métodos de predição da resposta para novos grupos poderiam ser desenvolvidos para outras classes de modelos com efeitos aleatórios como, por exemplo, os HGLM (hierarchical generalized linear models), propostos por Lee et al. (2006); os modelos mistos semi-paramétricos propostos por Lombardía e Sperlich (2012); e os modelos mistos com efeitos aleatórios temporais, apresentados por Estban e Santamaría (2012). Os HGLM são uma classe mais ampla do que os MLGM, permitindo que os efeitos aleatórios sigam outras distribuições além da normal multivariada; os modelos mistos semi-paramétricos permitem que os efeitos aleatórios possam seguir uma forma não-paramétrica; e modelos mistos com efeitos aleatórios temporais consideram a correlação temporal entre as observações dentro do mesmo grupo. 


\section{Apêndice A}

\section{Bloxplos dos estudos de simulação}

Neste Apêndice, são apresentados os resultados gráficos referentes aos estudos de simulação do Capítulo 4.

Cada cenário apresenta 4 figuras contendo os gráficos boxplots avaliados sob as 1.000 réplicas. A figura superior à esquerda apresenta os boxplots das medianas dos resíduos ao quadrado, a figura superior à direita apresenta os boxplots das medianas dos resíduos absolutos, a figura inferior à esquerda apresenta os boxplots dos AUC's e a figura inferior à direita apresenta os boxplots dos KS's. O eixo horizontal de cada figura está rotulado de v1 a v17, com base na seguinte legenda:

- v1 - modelo tradicional,

- v2 - modelo misto com método de estimação QVP e método de predição MPE,

- v3 - modelo misto com método de estimação QVP e método de predição RL,

- v4 - modelo misto com método de estimação QVP e método de predição NP,

- v5 - modelo misto com método de estimação QVP e método de predição MZ,

- v6 - modelo misto com método de estimação AL e método de predição MPE,

- v7 - modelo misto com método de estimação AL e método de predição RL,

- v8 - modelo misto com método de estimação AL e método de predição NP,

- v9 - modelo misto com método de estimação AL e método de predição MZ,

- v10 - modelo misto com método de estimação QAGH=2 e método de predição MPE,

- v11 - modelo misto com método de estimação QAGH=2 e método de predição RL,

- v12 - modelo misto com método de estimação QAGH=2 e método de predição NP,

- v13 - modelo misto com método de estimação QAGH=2 e método de predição MZ,

- v14 - modelo misto com método de estimação QAGH=5 e método de predição MPE,

- v15 - modelo misto com método de estimação QAGH=5 e método de predição RL, 
- v16 - modelo misto com método de estimação QAGH=5 e método de predição NP,

- v17 - modelo misto com método de estimação QAGH=5 e método de predição MZ. 

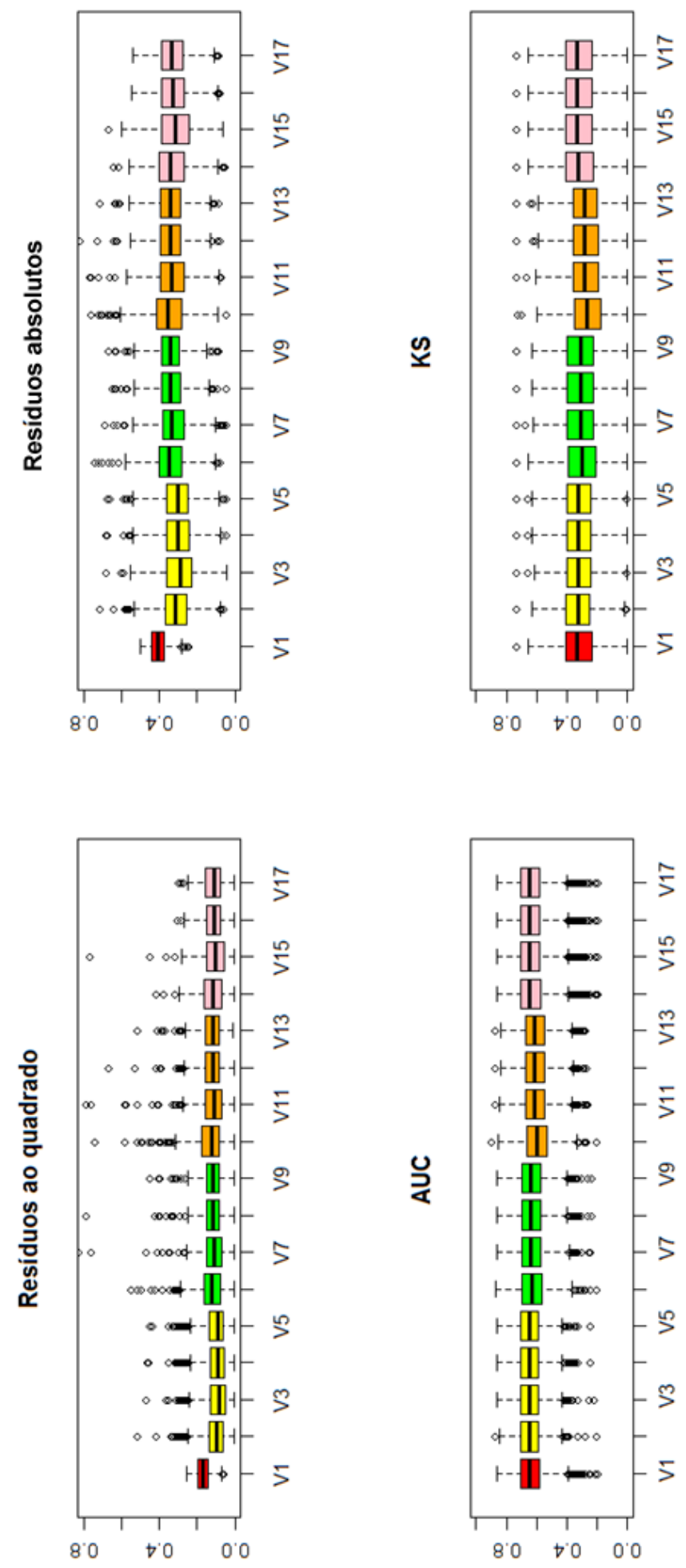

Figura A.1: Bloxplot das réplicas para Cenário 1 na base de predição: Distribuição das medianas dos resíduos ao quadrado, distribuição das medianas dos resíduos absolutos, distribuição do AUC e distribuição do KS. 

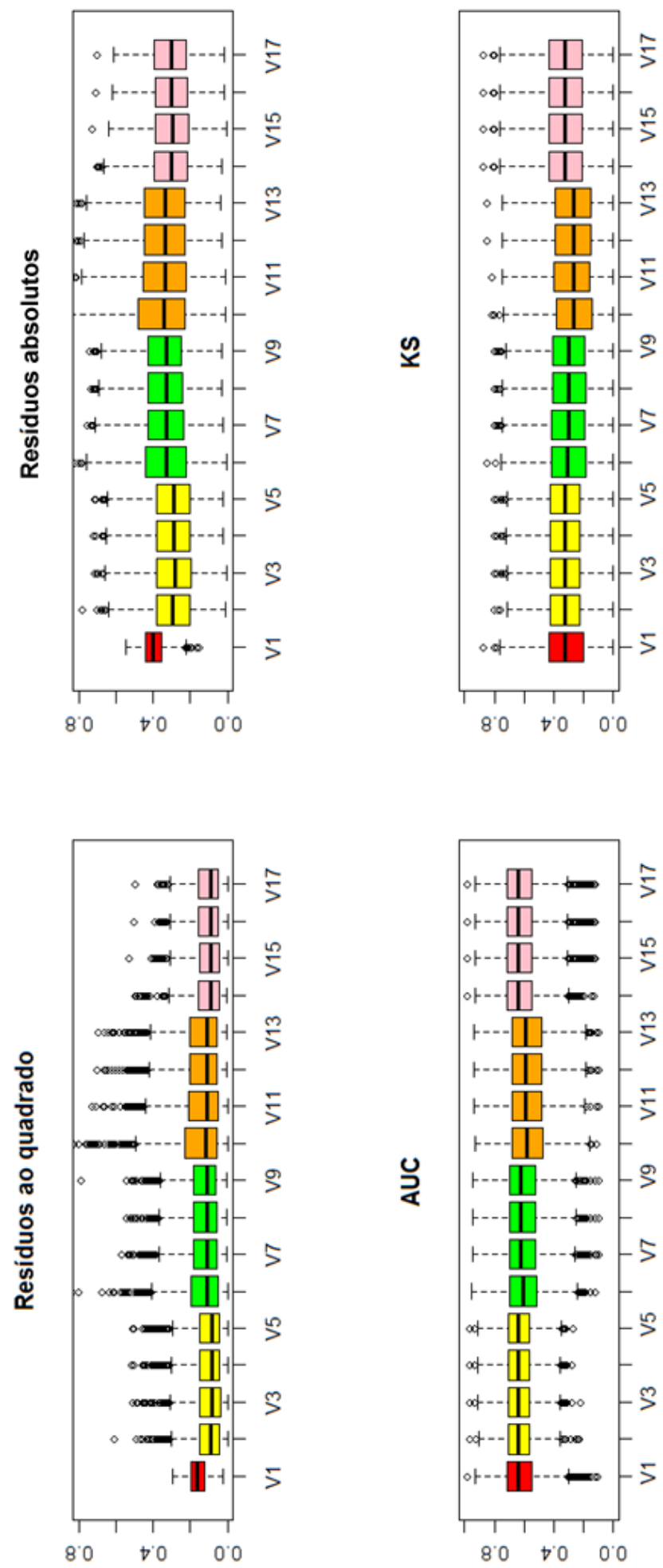

Figura A.2: Bloxplot das réplicas para Cenário 2 na base de predição: Distribuição das medianas dos resíduos ao quadrado, distribuição das medianas dos resíduos absolutos, distribuição do AUC e distribuição do KS. 

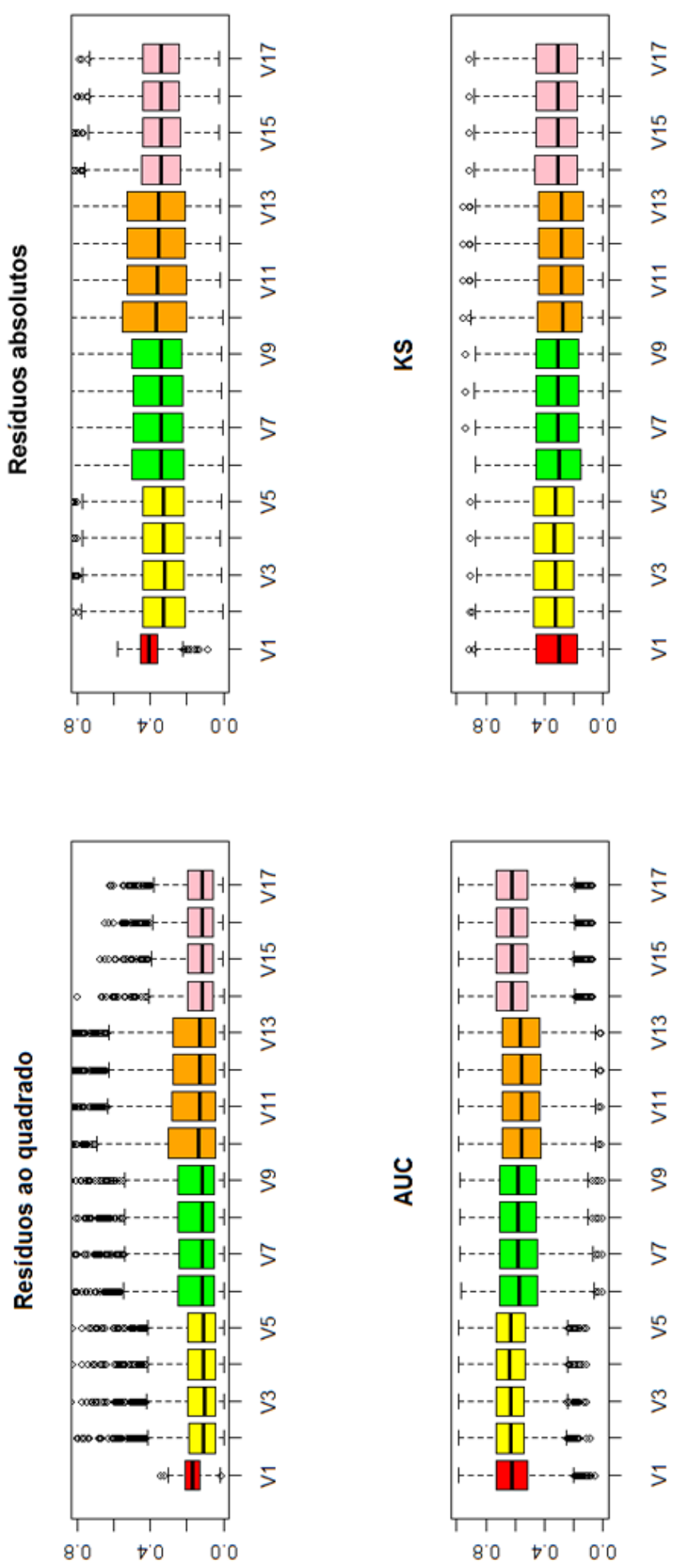

Figura A.3: Bloxplot das réplicas para Cenário 3 na base de predição: Distribuição das medianas dos resíduos ao quadrado, distribuição das medianas dos resíduos absolutos, distribuição do AUC e distribuição do KS. 

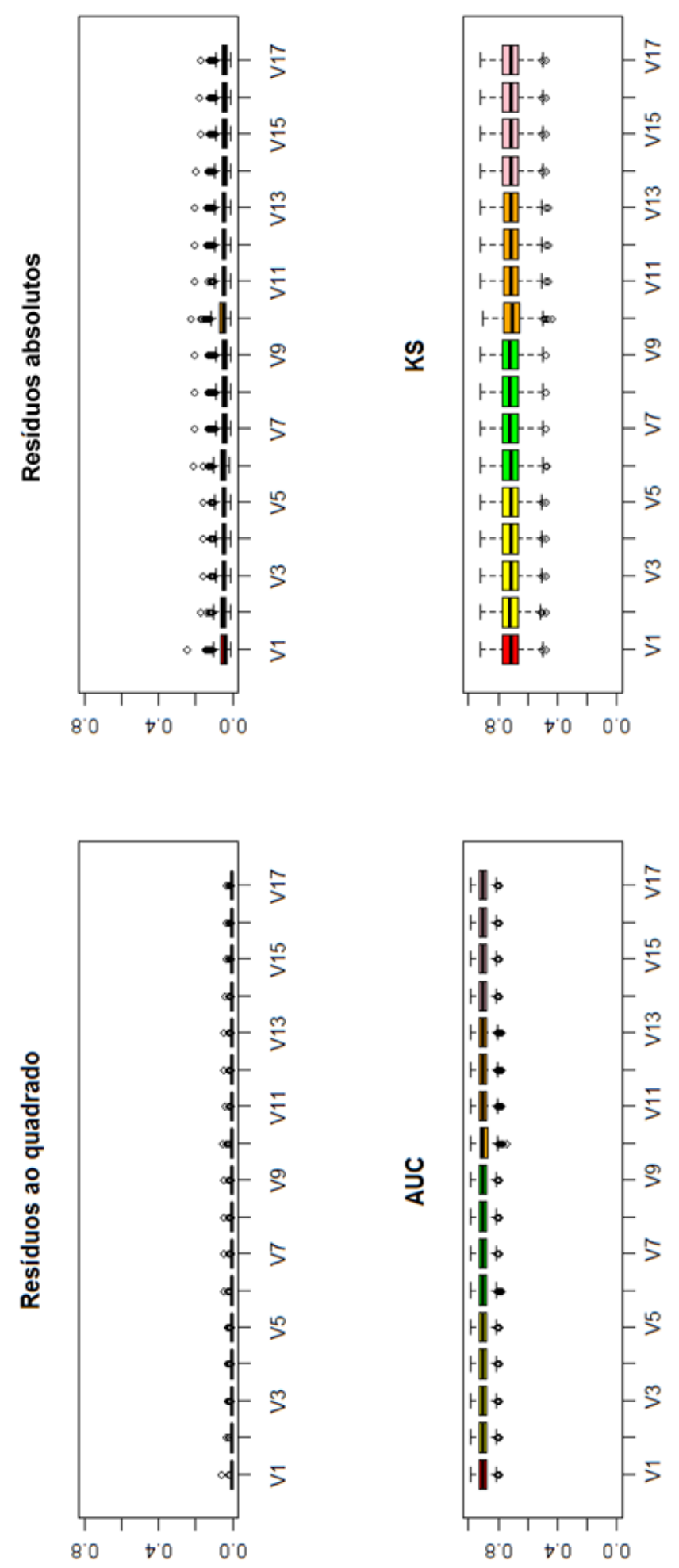

Figura A.4: Bloxplot das réplicas para Cenário 4 na base de predição: Distribuição das medianas dos resíduos ao quadrado, distribuição das medianas dos resíduos absolutos, distribuição do AUC e distribuição do KS. 

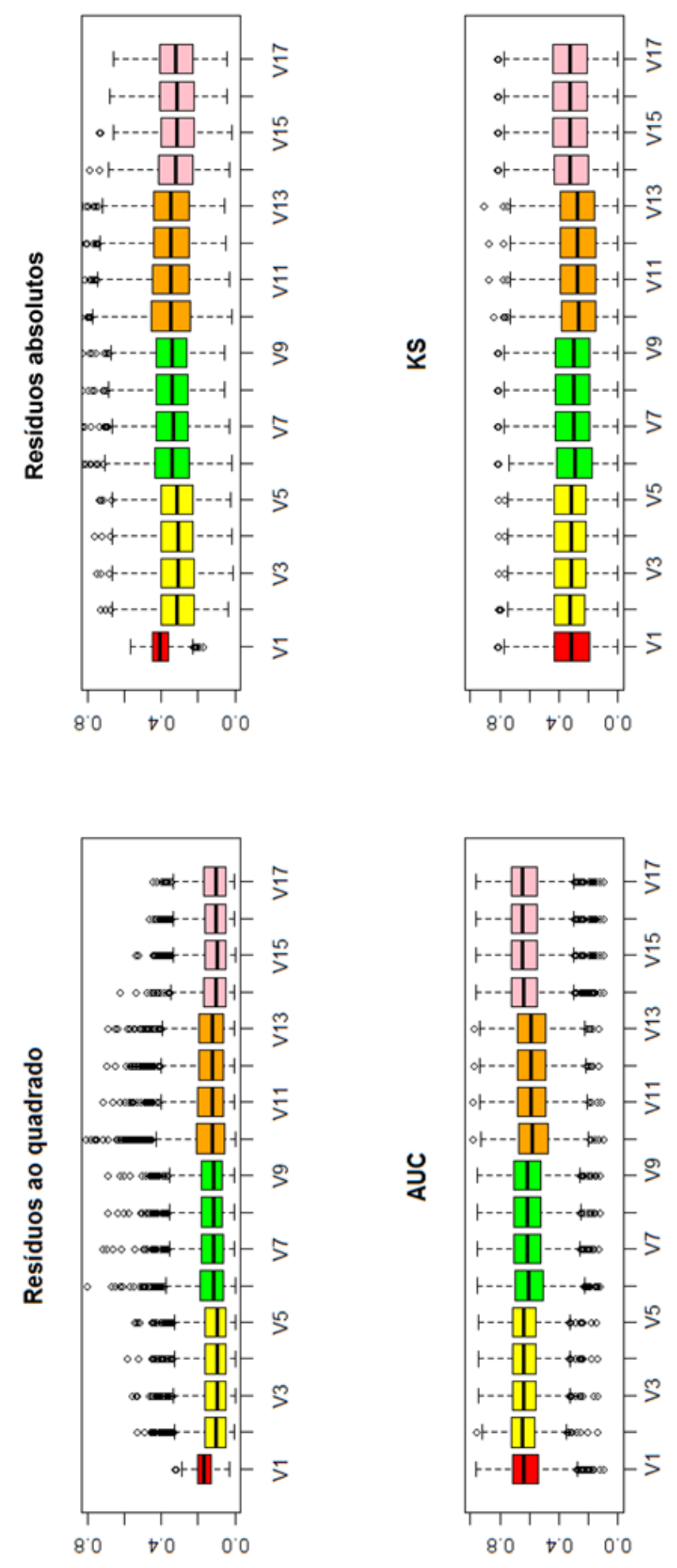

Figura A.5: Bloxplot das réplicas para Cenário 5 na base de predição: Distribuição das medianas dos resíduos ao quadrado, distribuição das medianas dos resíduos absolutos, distribuição do AUC e distribuição do KS. 

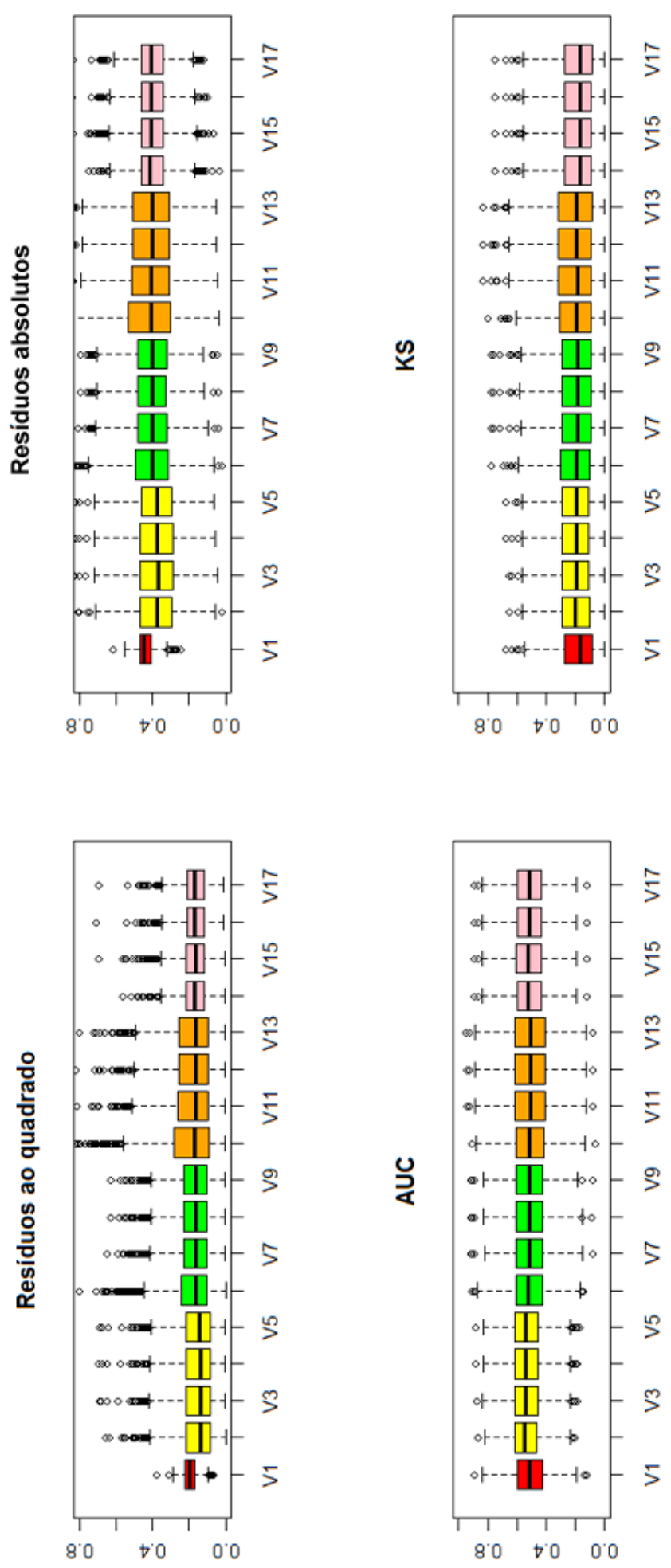

Figura A.6: Bloxplot das réplicas para Cenário 6 na base de predição: Distribuição das medianas dos resíduos ao quadrado, distribuição das medianas dos resíduos absolutos, distribuição do AUC e distribuição do KS. 

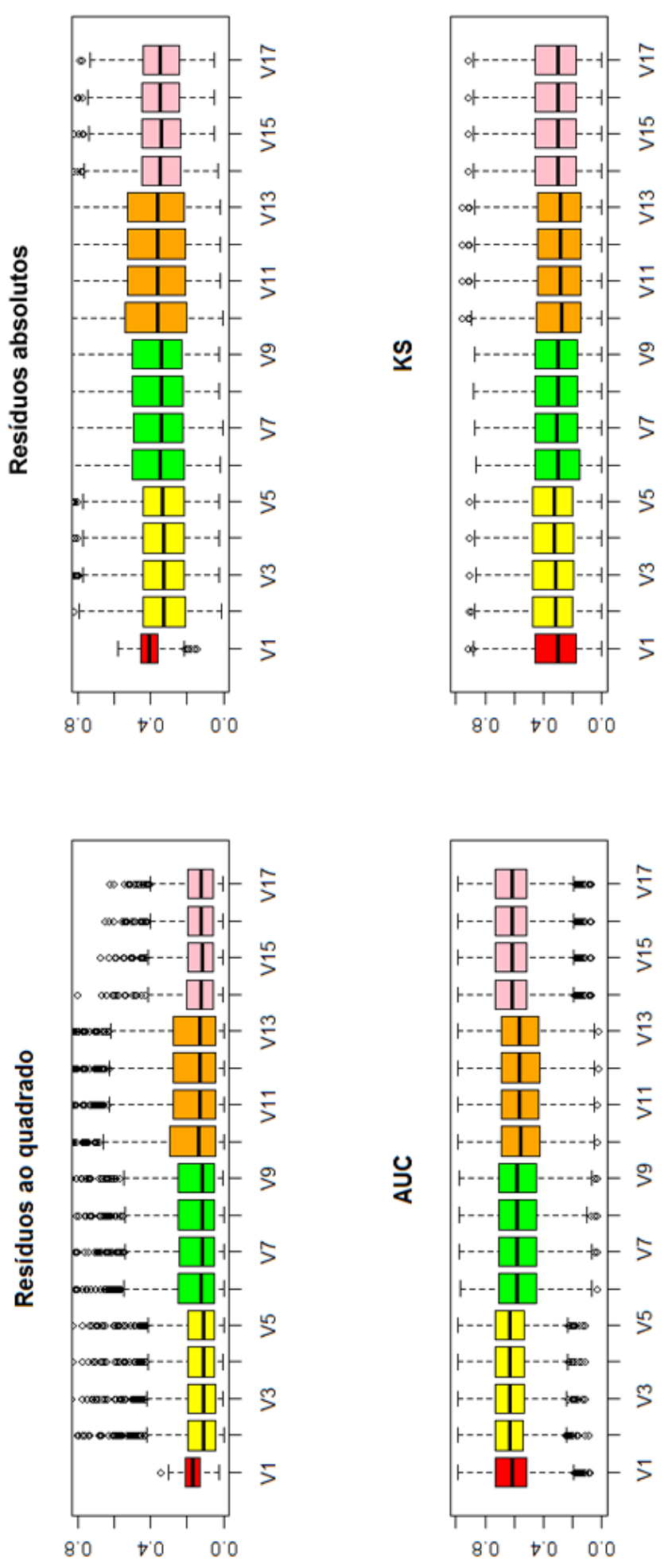

Figura A.7: Bloxplot das réplicas para Cenário 7 na base de predição: Distribuição das medianas dos resíduos ao quadrado, distribuição das medianas dos resíduos absolutos, distribuição do AUC e distribuição do KS. 


\section{Referências Bibliográficas}

Afshartous e de Leeuw (2005) D. Afshartous e J. de Leeuw. Prediction in multilevel models. Journal of Educational and Behavioral Statistics, 30(2):109-139. Citado na pág. 10

Albert (2012) P.S. Albert. A linear mixed model for predicting a binary event from longitudinal data under random effects misspecification. Statistics in Medicine. Citado na pág. 80

Alves (2008) M. C. Alves. Estratégias para o desenvolvimento de modelos de credit score com inferência de rejeitados. Dissertação de Mestrado, Instituto de Matemática e Estatística, Universidade de São Paulo, Brasil. Citado na pág. 19

Baayen et al. (2008) R.H. Baayen, D.J. Davidson e D.M. Bates. Mixed-effects modeling with crossed random effects for subjects and items. Journal of Memory and Language, 59(4):390-412. Citado na pág. 64

Bondeson (1990) J. Bondeson. Prediction in random coefficient regression models. Biometrical Journal, 32(4):387-405. Citado na pág. 10

Booth e Hobert (1999) P.G. Booth e J.P. Hobert. Maximizing generalized linear mixed model likelihood with an automated Monte Carlo EM algorithm. Journal of the Royal Statistical Society: Series B (Statistical Methodology), 61(1):265-285. Citado na pág. 23

Breslow e Clayton (1993) N.E. Breslow e D.G. Clayton. Approximate inference in generalized linear mixed models. Journal of the American Statistical Association, 88:9-25. Citado na pág. 23, 29,30

Butera (2000) A. M. Butera. Cross-selling: Capitalizing on the opportunities. Hoosier Bank, 87 (7):14-16. Citado na pág. 69

Candel (2004) M.J.J.M. Candel. Performance of empirical Bayes estimators of random coefficients in multilevel analysis: Some results for the random intercept-only model. Statistica Neerlandica, 58(2):197-219. Citado na pág. 10

Candel (2007) M.J.J.M. Candel. Empirical Bayes estimators of the random intercept in multilevel analysis: Performance of the classical, Morris and Rao version. Computational Statistics and Data Analysis, 51(6):3027-3040. Citado na pág. 10

Carlin e Louis (2000a) B.P. Carlin e T.A. Louis. Bayes and empirical Bayes methods for data analysis. Chapman \& Hall/CRC Press, Boca Raton, FL. Citado na pág. 11

Carlin e Louis (2000b) B.P. Carlin e T.A. Louis. Empirical Bayes: past, present and future. Journal of the American Statistical Association, 95(452):1286-1289. Citado na pág. 11

Cleveland (1979) W.S. Cleveland. Robust locally weighted regression and smoothing scatterplots. Journal of the American Statistical Association, 74(368):829-836. Citado na pág. 43 
Cole et al. (2003) D.J. Cole, B.J.T. Morgan e M.S. Ridout. Generalized linear mixed models for strawberry inflorescence data. Statistical Modelling, 3(4):273-290. Citado na pág. 23

Collett (2002) D. Collett. Modelling Binary Data. Chapman \& Hall, London, UK, second ed. Citado na pág. 17

Conover (1999) W.J. Conover. Practical Nonparametric Statistics. Wiley, New York, USA, second ed. Citado na pág. 19

Cook (2007) N.R. Cook. Use and misuse of the receiver operating characteristic curve in risk prediction. Circulation, 115(7):928-935. Citado na pág. 62

Demidenko (2004) E. Demidenko. Mixed Models: Theory and Applications. Wiley-Interscience, New York, USA. Citado na pág. 2, 22, 23

Draper et al. (1998) N.R. Draper, H. Smith e E. Pownell. Applied Regression Analysis. Wiley \& Sons, New York, USA, third ed. Citado na pág. 31, 39, 40

Efron e Morris (1973) B. Efron e C. Morris. Stein's estimation rule and its competitors an empirical Bayes approach. Journal of the American Statistical Association, 68(341):117-130. Citado na pág. 11

Efron e Morris (1975) B. Efron e C. Morris. Data analysis using Stein's estimator and its generalizations. Journal of the American Statistical Association, 70(350):311-319. Citado na pág. 11

Estban e Santamaría (2012) D. Pérez A. Estban, M.D. Morales e L. Santamaría. Small area estimation of poverty proportions under area-level time models. Computational Statistics and Data Analysis, 56(10):2840-2855. Citado na pág. 80

Fahrmeir et al. (1994) L. Fahrmeir, G. Tutz e W. Hennevogl. Multivariate Statistical Modelling Based on Generalized Linear Models. Springer, New York, USA, second ed. Citado na pág. 27, 44

Faraway (2002) J.J. Faraway. Practical Regression and ANOVA using R, 2002. Citado na pág. 31, 40

Fawcett (2006) T. Fawcett. An introduction to ROC analysis. Pattern Recognition Letters, 27 (8):861-874. Citado na pág. 16, 18

Fox (2000a) J. Fox. Nonparametric Simple Regression: Smoothing Scatterplots. Sage Publications, Thousand Oaks, CA. Citado na pág. 32, 40

Fox (2000b) J. Fox. Multiple and Generalized Nonparametric Regression. Sage Publications, Thousand Oaks, CA. Citado na pág. 32, 40

Frees e Kim (2006) E.W. Frees e J.S. Kim. Multilevel model prediction. Psychometrika, 71(1): 79-104. Citado na pág. 10

Goldstein (2011) H. Goldstein. Multilevel Statistical Models. Arnold Publishers, London, UK, fourth ed. Citado na pág. 1

Green (1987) P.J. Green. Penalized likelihood for general semi-parametric regression models. International Statistical Review, 55:245-259. Citado na pág. 29, 30 
Green e Silverman (1993) P.J. Green e B.W. Silverman. Nonparametric Regression and Generalized Linear Models: A Roughness Penalty Approach. Chapman \& Hall, London, UK. Citado na pág. 44

Hahn (2005) T. Hahn. Cuba - a library for multidimensional numerical integration. Computer Physics Communications, 168(2):78-95. Citado na pág. 59

Hanley e McNeil (1982) J.A. Hanley e B.J. McNeil. The meaning and use of the area under a receiver operating characteristic (ROC) curve. Radiology, 143:29-36. Citado na pág. 18

Harville (1976) D. Harville. Extension of the Gauss-Markov theorem to include the estimation of random effects. The Annals of Statistics, 4(2):384-395. Citado na pág. 10

Hastie e Tibshirani (1990) T. Hastie e R. Tibshirani. Generalized Additive Models. Chapman \& Hall, London, UK. Citado na pág. 32, 40, 41, 42, 59

Hocking (1976) R.R. Hocking. The analysis and selection of variables in linear regression. Biometrics, 32:1-49. Citado na pág. 31, 40

Hosmer e Lemeshow (2000) D. Hosmer e S. Lemeshow. Applied Logistic Regression. Wiley \& Sons, New York, USA, second ed. Citado na pág. 16, 17, 19

Hox (1995) J.J. Hox. Applied multilevel analysis. TT-publikaties, Amsterdam, Netherlands. Citado na pág. 1

Jiang (2007) J. Jiang. Linear and Generalized Linear Mixed Models and their Applications. Springer, New York, USA. Citado na pág. 23

Jiang e Lahiri (2001) J. Jiang e P. Lahiri. Empirical best prediction for small area inference with binary data. Annals of the Institute of Statistical Mathematics, 53(2):217-243. Citado na pág. $2,3,14,15,33,78$

Jiang e Lahiri (2006) J. Jiang e P. Lahiri. Mixed model prediction and small area estimation. Test, 15(1):1-96. Citado na pág. 11, 12

Kackar e Harville (1984) R.N. Kackar e D.A. Harville. Approximations for standard errors of estimators of fixed and random effects in mixed linear models. Journal of the American Statistical Association, 79(388):853-862. Citado na pág. 10

Kamakura et al. (2003) W.A. Kamakura, M. Wedel, F. De Rosa e J.A. Mazzon. Cross-selling through database marketing: A mixed data factor analyzer for data augmentation and prediction. International Journal of Research in marketing, 20(1):45-65. Citado na pág. 62, 69

Lee et al. (2006) Y. Lee, J.A. Nelder e Y. Pawitan. Generalized Linear Models with Random Effects: Unified Analysis via H-likelihood. Chapman \& Hall, London, UK. Citado na pág. 80

Liu e Pierce (1994) Q. Liu e D.A. Pierce. A note on Gauss-Hermite quadrature. Biometrika, 81 (3):624-629. Citado na pág. 23, 25, 26, 60

Lombardía e Sperlich (2012) J.M. Lombardía e S. Sperlich. A new class of semi-mixed effects models and its application in small area estimation. Computational Statistics and Data Analysis, 56:2903-2917. Citado na pág. 80 
Maritz e Lwin (1989) J.S. Maritz e T. Lwin. Empirical Bayes Methods. Chapman \& Hall, London, UK. Citado na pág. 11

McCullagh e Nelder (1989) P. McCullagh e J.A. Nelder. Generalized Linear Models. Chapman \& Hall, London, UK, second ed. Citado na pág. 2, 8, 28

McCulloch (1997) C.E. McCulloch. Maximum likelihood algorithms for generalized linear mixed models. Journal of the American statistical Association, 92(437):162-170. Citado na pág. 23

McCulloch e Neuhaus (2011) C.E. McCulloch e J.M. Neuhaus. Prediction of random effects in linear and generalized linear models under model misspecification. Biometrics, 67(1):270-279. Citado na pág. 11, 36, 80

Metz (1978) C.E. Metz. Basic principles of ROC analysis. Em Seminars in Nuclear Medicine, volume 8, páginas 283-298. Elsevier. Citado na pág. 18

Molenberghs e Verbeke (2005) G. Molenberghs e G. Verbeke. Models for Discrete Longitudinal Data. Springer, New York, USA. Citado na pág. 28

Morris (1983) C.N. Morris. Parametric empirical Bayes inference: Theory and applications. Journal of the American Statistical Association, 78(381):47-55. Citado na pág. 11

Neter et al. (1996) J. Neter, W. Wasserman e M.H. Kutner. Applied Linear Regression Models. Irwin, Homewood, IL, third ed. Citado na pág. 31, 40

Papoila (2012) A.L. Papoila. Modelos de predição em Medicina: Algumas considerações. Métodos Estatísticos em Medicina, páginas 26-35. Citado na pág. 62

Paula (2004) G.A. Paula. Modelos de Regressão: com Apoio Computacional. IME-USP, São Paulo, Brasil. Citado na pág. 8, 9

Pencina et al. (2008) M.J. Pencina, R.B. D'Agostino Sr, R.B. D'Agostino Jr e R.S. Vasan. Evaluating the added predictive ability of a new marker: From area under the ROC curve to reclassification and beyond. Statistics in Medicine, 27(2):157-172. Citado na pág. 62

Pinheiro e Bates (1995) J.C. Pinheiro e D.M. Bates. Approximations to the log-likelihood function in the nonlinear mixed-effects model. Journal of Computational and Graphical Statistics, 4(1):12-35. Citado na pág. 50

Pinheiro e Bates (2000) J.C. Pinheiro e D.M. Bates. Mixed-effects Models in $S$ and S-PLUS. Springer-Verlag, New York, USA. Citado na pág. 2

Pinheiro e Chao (2006) J.C. Pinheiro e E.C. Chao. Efficient laplacian and adaptive gaussian quadrature algorithms for multilevel generalized linear mixed models. Journal of Computational and Graphical Statistics, 15(1):58-81. Citado na pág. 50

Prasad e Rao (1990) N.G.N. Prasad e J.N.K. Rao. The estimation of the mean squared error of small-area estimators. Journal of the American Statistical Association, 85(409):163-171. Citado na pág. 2

Rabe-Hesketh et al. (2002) S. Rabe-Hesketh, A. Skrondal e A. Pickles. Reliable estimation of generalized linear mixed models using adaptive quadrature. The Stata Journal, 2(1):1-21. Citado na pág. 23,60 
Rao (1975) C.R. Rao. Simultaneous estimation of parameters in different linear models and applications to biometric problems. Biometrics, 31:545-554. Citado na pág. 10

Raudenbush e Bryk (2001) S.W. Raudenbush e A.S. Bryk. Hierarchical Linear Models: Applications and Data Analysis Methods. Sage Publications, Newbury Park, CA, second ed. Citado na pág. 1

Raudenbush et al. (2000) S.W. Raudenbush, M.L. Yang e M. Yosef. Maximum likelihood for generalized linear models with nested random effects via high-order, multivariate laplace approximation. Journal of computational and Graphical Statistics, 9(1):141-157. Citado na pág. 25, 30

Reinsch (1967) C.H. Reinsch. Smoothing by spline functions. Numerische Mathematik, 10(3): 177-183. Citado na pág. 44

Reinsel (1984) G. C. Reinsel. Estimation and prediction in a multivariate random effects generalized linear model. Journal of the American Statistical Association, 79(386):406-414. Citado na pág. 10

Reinsel (1985) G.C. Reinsel. Mean squared error properties of empirical Bayes estimators in a multivariate random effects general linear model. Journal of the American Statistical Association, 80(391):642-650. Citado na pág. 10

Renard (2002) D. Renard. Topics in Modeling Multilevel and Longitudinal Data. Tese de Doutorado, Limburgs Universitair Centrum. Citado na pág. 2

Rencher (1998) A.C. Rencher. Multivariate Statistical Inference and Applications. Wiley \& Sons, New York, USA. Citado na pág. 34

Robbins e Neyman (1956) H. Robbins e J. Neyman. An empirical Bayes approach to statistics. University of California Press Berkeley, CA. Citado na pág. 11

Robinson (1991) G.K. Robinson. That BLUP is a good thing: The estimation of random effects. Statistical Science, 6(1):15-51. Citado na pág. 11

Rodriguez e Goldman (1995) G. Rodriguez e N. Goldman. An assessment of estimation procedures for multilevel models with binary responses. Journal of the Royal Statistical Society. Series A (Statistics in Society), páginas 73-89. Citado na pág. 30

Rosenberg (1973) B. Rosenberg. Linear regression with randomly dispersed parameters. Biometrika, 60(1):65-72. Citado na pág. 10

Rosset et al. (2001) S. Rosset, E. Neumann, U. Eick, N. Vatnik e I. Idan. Evaluation of prediction models for marketing campaigns. Em Proceedings of the seventh ACM SIGKDD international conference on Knowledge discovery and data mining, páginas 456-461. ACM. Citado na pág. 62

Searle et al. (1992) S.R. Searle, G. Casella, C.E. McCulloch et al. Variance Components. Wiley \& Sons, New York, USA. Citado na pág. 11

Skrondal e Rabe-Hesketh (2009) A. Skrondal e S. Rabe-Hesketh. Prediction in multilevel generalized linear models. Journal of the Royal Statistical Society: Series A, 172(3):659-687. Citado na pág. 2, 11, 32, 78 
Song et al. (2005) P.X.K. Song, Y. Fan e J.D. Kalbfleisch. Maximization by parts in likelihood inference. Journal of the American Statistical Association, 100(472):1145-1158. Citado na pág. 23

Steyerberg et al. (2010) E.W. Steyerberg, A.J. Vickers, N.R. Cook, T. Gerds, M. Gonen, N. Obuchowski, M.J. Pencina e M.W. Kattan. Assessing the performance of prediction models: A framework for traditional and novel measures. Epidemiology, 21(1):128. Citado na pág. 62

Strenio et al. (1983) J.F. Strenio, H.I. Weisberg e A.S. Bryk. Empirical Bayes estimation of individual growth-curve parameters and their relationship to covariates. Biometrics, 39:71-86. Citado na pág. 10

Swamy (1970) P. Swamy. Efficient inference in a random coefficient regression model. Econometrica, 38:311-323. Citado na pág. 10

Swets et al. (1979) J.A. Swets, R.M. Pickett, S.F. Whitehead, D.J. Getty, J.A. Schnur, J.B. Swets, B.A. Freeman et al. Assessment of diagnostic technologies. Science, 205(4408):753-759. Citado na pág. 18

Tamura (2007) K.A. Tamura. Modelo logístico multinível: Um enfoque em métodos de estimação e predição. Dissertação de Mestrado, Instituto de Matemática e Estatística, Universidade de São Paulo, Brasil. Citado na pág. 30

Tamura e Giampaoli (2010) K.A. Tamura e V. Giampaoli. Prediction in multilevel logistic regression. Communications in Statistics-Simulation and Computation, 39(6):1083-1096. Citado na pág. $2,3,11,14,15,31,33,36,74,78$

Team (2010) R Development Core Team. R version 2.10.1. http://cran.r-roject.org/bin/ windows/base/old/2.10.1/, 2010. Citado na pág. 59

Timm (2002) N.H. Timm. Applied multivariate analysis. Springer, New York, USA. Citado na pág. 34

Tutz (2011) G. Tutz. Regression for Categorical Data. Cambridge University Press, New York, USA. Citado na pág. 24, 26, 27

Ware e Wu (1981) J.H. Ware e M.C. Wu. Tracking: Prediction of future values from serial measurements. Biometrics, páginas 427-437. Citado na pág. 10

Weisberg e Fox (2010) S. Weisberg e J. Fox. An $R$ companion to Applied Regression. Sage Publications, Thousand Oaks, CA. Citado na pág. 32, 40

Youden (1950) W.J. Youden. Index for rating diagnostic tests. Cancer, 3(1):32-35. Citado na pág. 20

Zeger e Karim (1991) S.L. Zeger e M.R. Karim. Generalized linear models with random effects; a Gibbs sampling approach. Journal of the American Statistical Association, 86(413):79-86. Citado na pág. 23 